\title{
A New Generic Maximum-Likelihood Metric Expression for Space-Time Block Codes With Applications to Decoding
}

by

\section{JefF LeUSCHNER}

\author{
A thesis submitted to the \\ Department of Electrical and Computer Engineering \\ in conformity with the requirements for \\ the degree of Master of Science (Engineering)
}

Queen's University

Kingston, Ontario, Canada

August 2007

Copyright (C) Jeff Leuschner, 2007 


\section{Abstract}

Space-time block coding is a technique used to exploit diversity in a multiple-input multiple-output (MIMO) environment. Orthogonal space-time block codes (OSTBCs) are desirable because they can achieve full transmit diversity while maintaining a simple low-complexity maximum-likelihood (ML) decoding algorithm. However, OSTBCs are limited in their error performance. This has led to the development of more general linear space-time block codes, such as quasi-orthogonal space-time block codes (QOSTBCs). QOSTBCs offer better error performance, but their decoding complexity is a concern since it is no longer a linear function of the number of transmitted symbols.

In this thesis, a new vectorization for linear STBCs is proposed that explicitly maintains the redundancy in the STBC transmission matrix. By expressing the ML metric using the new vectorization, a new generic representation of the ML metric expression for a linear STBC is derived. One immediate application of this new metric expression is the convenient partial decoupling and simplification of the detection metric for linear STBCs.

The new metric expression can also be used as a design tool to help in the construction of new STBCs with low decoding complexity. As an example, a new QOSTBC is constructed that has lower decoding complexity than one previously proposed in 
the literature of equal rate and diversity.

A comparison is conducted to answer the following question: for the family of QOSTBCs, when is it best to perform an exhaustive search using a metric expression that is simplified and decoupled as much as possible, and when should an efficient implementation of the sphere decoding algorithm be applied? Determining this boundary is an important and practical issue not yet directly addressed in the literature.

The new metric expression can also be used as the framework for a new family of sub-optimal decoding algorithms for STBCs that trade-off error performance for a reduction in decoding complexity. A practical example of such an algorithm is given as an example. 


\section{Acknowledgments}

I am grateful to my advisor, Professor Shahram Yousefi, for supervising my research over the last two years. The time he set aside to provide advice, direction, and encouragement was invaluable. His positive attitude and passion for the subject was the inspiration that kept me motivated, and his flexibility made the program go by smoothly. His feedback during our research meetings showed that he took the time to carefully review and ponder my work. I benefited from his qualities as a teacher and mentor.

I would also like to thank the Natural Sciences and Engineering Research Council (NSERC) of Canada for providing the majority of the funding needed to conduct my

research. It is great to live in a country that realizes the importance of encouraging research and innovation.

Finally, I would like to thank the faculty and students I have interacted with during my research and course work. Our discussions have allowed me to comprehend the area more fully. Also, thank you to the support staff for making everything flow smoothly. 


\section{Table of Contents}

Abstract

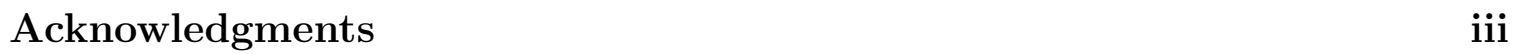

Table of Contents iv

List of Tables $\quad$ vi

List of Figures vii

Chapter 1:

Fundamentals ................... 1

1.1 Introduction . . . . . . . . . . . . . . . . . . . . . . 1

$1.2 \quad$ A Digital Communication System . . . . . . . . . . . . . . . 2

1.3 An Overview of the Wireless Channel . . . . . . . . . . . . . . . . . . 4

$1.4 \quad$ Multiple-Input Multiple-Output Channels . . . . . . . . . . . . . . . 8

1.5 MIMO Decoding Algorithms . . . . . . . . . . . . . . . . . . . . . . . 18

1.6 Conclusion . . . . . . . . . . . . . . . . . . . . . 25

Chapter 2:

Alternative Vectorization for a Linear STBC . . . . . 26 
2.1 Introduction . . . . . . . . . . . . . . . . . . . 26

2.2 An Alternative Vectorization . . . . . . . . . . . . . . . . . . 27

$2.3 \quad$ Expanded ML Metric $\ldots \ldots \ldots \ldots \ldots \ldots$

2.4 Examples $\ldots \ldots \ldots \ldots \ldots$

2.5 Conclusion $\ldots \ldots \ldots \ldots \ldots \ldots \ldots$

Chapter 3:

ML and sub-ML Decoding of QOSTBCs $\ldots \ldots \ldots$

3.1 Introduction $\ldots \ldots \ldots \ldots \ldots$

$3.2 \quad$ ML Decoding Algorithms for STBCs: SDA versus an Exhaustive Search 39

3.3 Sub-optimal Decoding Algorithms . . . . . . . . . . . . . . . . . 44

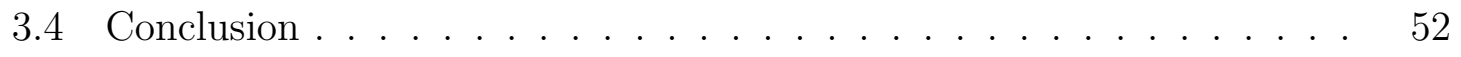

Chapter 4:

Future Work $\ldots \ldots \ldots \ldots \ldots \ldots \ldots$

4.1 Introduction $\ldots \ldots \ldots \ldots \ldots \ldots \ldots$

4.2 Optimum Rotation Angle for the New QOSTBC $\ldots \ldots \ldots \ldots \ldots$

4.3 Development of Optimality Test Criteria For STBCs $\ldots \ldots \ldots$

4.4 Conclusions $\ldots \ldots \ldots \ldots$

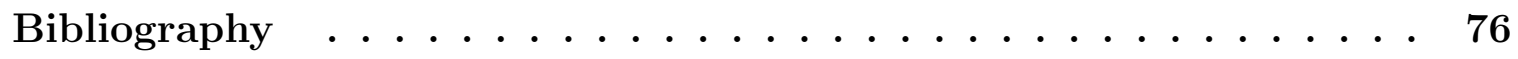




\section{List of Tables}

3.1 A complexity comparison (in terms of number of flops per information symbol) among zero-forcing, an exhaustive search, and the sphere decoding algorithm for the QOSTBCs in [11]. . . . . . . . . . . . . . 46

3.2 A complexity comparison (in terms of real multiplications/additions) for each $n$ value. Rayleigh fading is assumed, and the QOSTBC is that constructed in $(2.5)$ with 16-QAM. . . . . . . . . . . . . . . 52 


\section{List of Figures}

1.1 The functional block diagram of a communication system. . . . . . . 3

1.2 The basic elements of a digital communication system. . . . . . . . . 4

1.3 The continuous-time bandpass model for a wireless channel using a linear time-varying filter with an additive noise component. . . . . . . 6

1.4 The 4-QAM and 16-QAM constellations. . . . . . . . . . . . . . . . 8

1.5 $\quad$ By using multiple transmit and receive antennas, $x$ can be sent along multiple paths in the channel to achieve better error performance. . . $\quad 9$

1.6 Geometric interpretation of the SDA. . . . . . . . . . . . . . . . . 21

2.1 SER for $n_{T}=8$, rate-one QOSTBCs with 4-QAM, $n_{R}=1$, and Rayleigh fading. (a) QOSTBC proposed in [11] with its optimum constellation rotation angles $\theta=\pi / 6$ and $\theta^{\prime}=\pi / 4$ (see [11, eqn. (7)]). (b) New QOSTBC constructed (2.5) where $x_{5}, x_{6}, x_{7}$, and $x_{8}$ are drawn from a constellation rotated by $\pi / 8$. . . . . . . . . . . . . . . . . . . . 37

3.1 Complexity comparison for $n_{T}=8$, rate-one QOSTBC in [11] for a Rayleigh fading channel. (a) 4-QAM. (b) 16-QAM. . . . . . . . . . . 42

$3.2 \quad$ Complexity comparison for $n_{T}=8$, rate-one QOSTBC constructed in (2.5) for a Rayleigh fading channel. (a) 4-QAM. (b) 16-QAM. . . . . 43 
3.3 A BER comparison of ML decoding versus zero-forcing for the 4 and 8 antenna QOSTBCs proposed in [11]. Rayleigh fading is assumed and the underlying constellation is 16-QAM. . . . . . . . . . . . . . . . . 47

$3.4 \quad$ A generic framework for a near-ML decoding algorithm for non-orthogonal STBCs. . . . . . . . . . . . . . . . . . . . 48

3.5 An algorithm that uses $n$ to trade-off error performance for a reduction in complexity for the QOSTBC in (2.5) with 16-QAM. . . . . . . . . 50

3.6 A BER comparison of sub-optimal versus ML for the QOSTBC constructed in (2.5). Rayleigh fading is assumed and the underlying constellation is 16-QAM. . . . . . . . . . . . . . . . . . . . . 51

4.1 Partition of codeword positions. . . . . . . . . . . . . . . 65 


\section{Chapter 1}

\section{Fundamentals}

\section{$1.1 \quad$ Introduction}

In this introductory chapter, the fundamental concepts necessary to understand the contribution of this thesis will be reviewed. First, a single-user digital communication system with a wireless channel model will be reviewed. The introduction of diversity gain by adding multiple transmit and receive antennas will then be discussed, leading to the concept of a Multiple-Input Multiple-Output (MIMO) system. An overview of the system model and decoding metric for a MIMO system will then be presented. The chapter will conclude with a discussion of MIMO encoding and decoding schemes, including a review of the sphere decoding algorithm. Throughout this chapter, any assumptions made about the system model will hold for the rest of the thesis, unless otherwise stated. These assumptions will be summarized again prior to the analysis at the beginning of chapter 2 .

The following notations are used in this chapter and throughout the rest of the thesis: bolded capital letters (e.g. $\mathbf{H})$ refer to matrices and bolded small letters (e.g. 
h) refer to vectors. $[\cdot]^{T}$ refers to the transpose of a matrix or vector. $\Re\{\cdot\}$ and $\Im\{\cdot\}$ refer to the real and imaginary components of a complex number. $\|\cdot\|^{2}$ refers to the squared 2-norm of a matrix or vector and $|\cdot|^{2}$ refers to the squared magnitude of a complex number. For a given $n \times m$ (in general complex) matrix $\mathbf{C}, \mathbf{c}_{j}$ indicates the $j^{\text {th }}$ column vector, and $\mathbf{c}^{(j)}$ indicates the $j^{\text {th }}$ row vector. $\operatorname{vec}(\mathbf{C})$ indicates that the matrix has been vectorized (i.e., $\operatorname{vec}(\mathbf{C})=\left[\begin{array}{llll}\mathbf{c}_{1}^{T} & \mathbf{c}_{2}^{T} \ldots & \mathbf{c}_{L}^{T}\end{array}\right]^{T}$ ). The squared Frobenius norm is $\|\mathbf{C}\|^{2}=\sum_{i=1}^{n} \sum_{j=1}^{m}\left|c_{i j}\right|^{2}$, which reduces to the squared Euclidean norm when either $m=1$ or $n=1$ and the entities are real. The inner product of two vectors is denoted by $\langle\mathbf{x}, \mathbf{y}\rangle=\sum_{i} x_{i} y_{i}^{*}$ where $*$ represents the complex conjugate of a number. $\otimes$ represents the Kronecker product, $\mathbf{I}_{L}$ is the $L \times L$ identity matrix, and $\mathcal{I}_{L}=\{1,2, \ldots, L\}$.

\section{$1.2 \quad$ A Digital Communication System}

The fundamental challenge of data communication systems is to efficiently transmit information from one point to another while maintaining a tolerable amount of error. Consider the basic model shown in Figure 1.1. The transmitter converts the source information into a form that is suitable for transmission over the channel. In general, it performs the matching of the message signal to the channel by a process called modulation. The channel is the medium that connects the transmitter and the receiver. In wireless communications, it is usually free space, and is modeled mathematically using a linear time-varying filter, along with an additive noise component. The channel introduces error into the information transmitted; it is the receiver's job to recover the signal (through demodulation and decoding) in a way that minimizes the effect of this error. In wireless communications, this noise may be from physical objects in the environment, thermal noise, or electromagnetic signals from other sources (e.g. other 


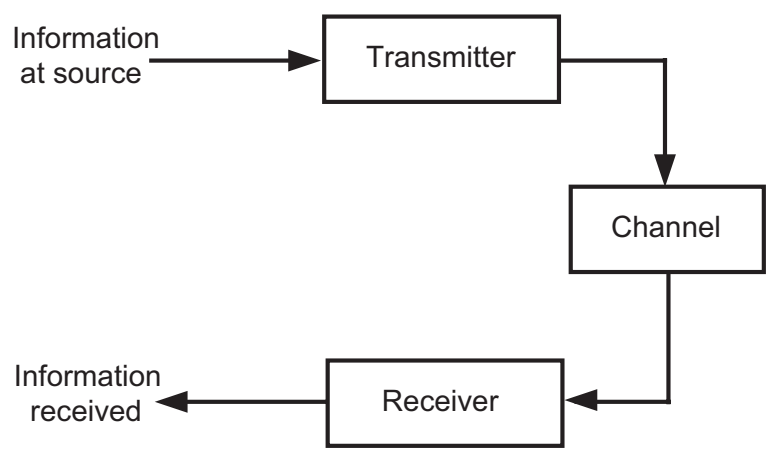

Figure 1.1: The functional block diagram of a communication system.

users in multiple access systems). If the information source is in digital form, then the system in Figure 1.1 is classified as a digital communication system. Digital communication systems have a number of advantages; notably, they allow for repeaters over long transmission distances, and they permit source compression, error control coding, and digital signal processing of many forms.

The basic elements of a digital communication system are shown in Figure 1.2 . The source encoder performs data compression to minimize the redundancy in the information sequence. The channel encoder then introduces, in a controlled manner, redundancy in the information sequence that is used at the receiver to overcome the effects of noise and interference encountered in the channel. The digital modulator then maps the encoded information sequence to analog signal waveforms appropriate for transmission across the channel. 


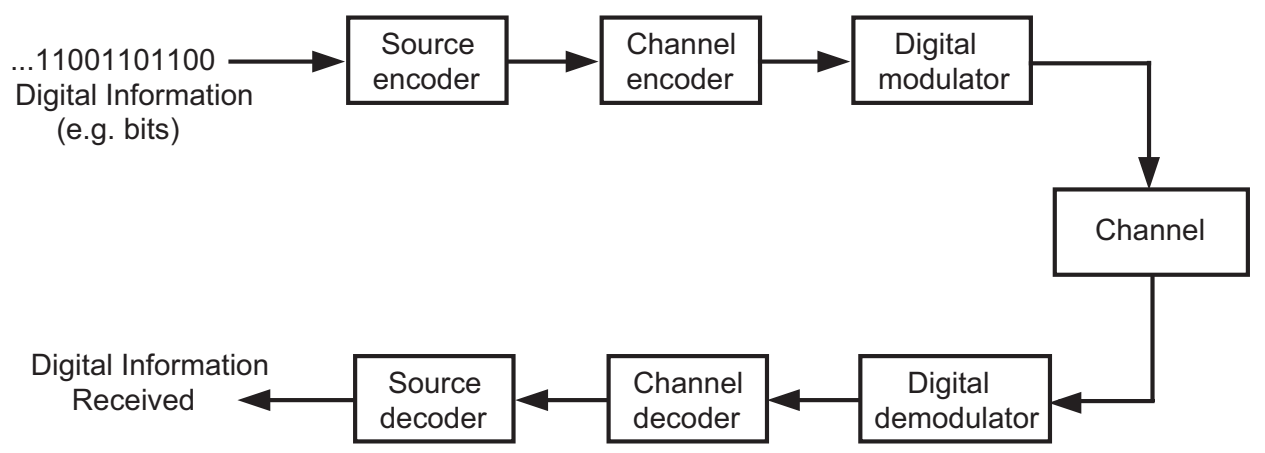

Figure 1.2: The basic elements of a digital communication system.

\subsection{An Overview of the Wireless Channel}

The wireless channel presents a number of challenges that must be accounted for in a mathematical model. Assume the digital communication system in Figure 1.2 , in which a single user is transmitting information from one transmit antenna to one receive antenna through a wireless channel. For each group of symbols mapped to the digital modulator, a corresponding electromagnetic wave will be transmitted into the channel via the transmit antenna. It disperses and travels along multiple paths before being received by the receive antenna. As a result, the receiver will receive multiple copies of the transmitted signal with different levels of power over a time window. This delay spread caused by the channel suggests that inter-symbol interference (ISI) may be a problem, i.e., that adjacent transmitted symbols may interfere with each other during transmission. A wireless channel in which ISI occurs is referred to as frequency selective; otherwise, the channel is referred to as frequency flat. Whether a channel is characterized as frequency selective or frequency flat depends on the delay spread in the channel compared to the symbol duration. The delay spread or corresponding coherence bandwidth can be defined and compared 
against the symbol duration or signal bandwidth to characterize the channel. Often ISI can be eliminated (or minimized enough) by using orthogonal frequency-division multiplexing or equalization techniques. Therefore, much analyses performed on the wireless channel assume it is frequency flat. The rest of this thesis will consider only channels that are frequency flat.

Another challenge presented by the wireless channel is caused by the movement of transmit and receive antennas, which results in a doppler shift of the transmitted signal. The doppler shift causes periodic power fluctuations in the signal. It is undesirable to receive the transmitted signal during a period of deep attenuation (referred to as a deep fade). It is also problematic if the transmitted signal moves into or out of a deep fade during the transmission of a single symbol. The coherence time of a channel refers to the length of time the channel stays relatively constant. If the symbol duration is less than the coherence time, the channel is referred to as slow fading; otherwise, the channel is referred to as fast fading. A quasi-static channel is a special case of a slow fading channel, and will be discussed later. The rest of this thesis will consider only channels that are slow/quasi-static fading.

\subsubsection{Channel Model}

Given the challenges discussed above, how can one formulate a tractable mathematical model for the wireless channel? If we assume the channel is linear, the delay spread and multiplicative fading can be simply modeled using a linear time-varying filter. Specifically, given $c(t)$ is transmitted, the receiver receives $r(t)$ as shown in Figure 1.3. 


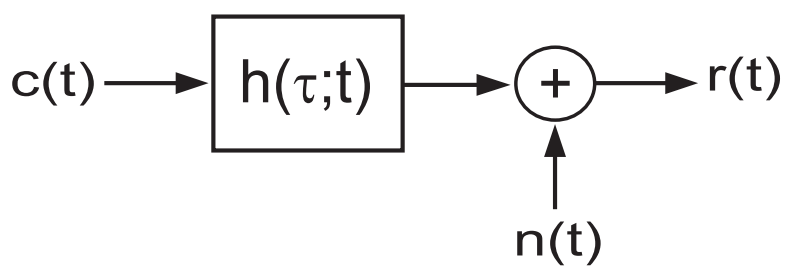

Figure 1.3: The continuous-time bandpass model for a wireless channel using a linear time-varying filter with an additive noise component.

$$
\begin{aligned}
r(t) & =h(\tau ; t) * c(t)+n(t) \\
& =\sum_{i} a_{i}(t) c\left(t-\tau_{i}(t)\right)+n(t)
\end{aligned}
$$

where $i$ represents the number of paths $c(t)$ traverses, $a_{i}(t)$ represents the multiplicative gain for each path, and $\tau_{i}(t)$ represents the delay of each path. $n(t)$ is the additive noise component. The additive component is often assumed to be white and zero-mean Gaussian.

The above model is continuous-time, and $c(t)$ may be a bandpass signal in general. In analysis, it is usually more convenient to work with the equivalent discrete-time baseband model. The derivation of this equivalent model is outlined in detail in [1]. Using the discrete-time baseband model and assuming both a frequency flat and slow fading channel, the received complex number at time slot $t, r[t]$, is as follows:

$$
r[t]=h \times c[t]+n[t]
$$

where $c[t]$ is the underlying constellation symbol, $h$ is the multiplicative noise factor, and $n[t]$ is the additive noise component. $h$ can be modeled as suited for the channel, but it is common to assume an infinite number of multipaths and use the central-limit theorem to model $h$ as a circular symmetric complex Gaussian random variable. This fading model is referred to as Rayleigh fading (if the random variable is zero-mean) 
or Ricean fading (if the random variable is not zero-mean). Assuming the additive noise is white and Gaussian, $n[t]$ is a circular symmetric complex Gaussian random variable with variance $\frac{N_{o}}{2}$, where $N_{o}$ is the one-sided power spectral density of the noise. Without loss of generality, the time notation will be dropped. Therefore, at each time slot, $r=h c+n$ is received. Based on the value $r$ received and any channel state information (CSI) available, the decoder will make a guess as to what constellation symbol, $c$, was transmitted.

\subsubsection{Constellations}

When working with the discrete-time baseband model, the signal sent through the channel, $c$, is represented by a complex number. The magnitude of $c$ corresponds to the energy of the sinusoid, and the phase of $c$ corresponds to the phase of the sinusoid. $c$ is referred to as a constellation symbol, and the set of all possible constellation symbols that may be sent make up the underlying constellation. Deciding what underlying constellation to use amounts to deciding how many bits of information each signal represents. For example, the 4-QAM constellation represents two bits for each constellation symbol, whereas the 16-QAM constellation represents four bits for each constellation symbol (see Figure 1.4). Other popular constellations include BPSK, 8-PSK, and 64-QAM.

For a given average energy spending, there is a direct trade-off between bandwidth and error performance. For example, 16-QAM offers better bandwidth efficiency than 4-QAM (since one symbol sent through the channel represents four information bits), but the error performance is worse since the distance between the constellation symbols is smaller (i.e., the signals are "closer" in the sense that the channel is more 
likely to affect one signal such that it is mistaken as another).
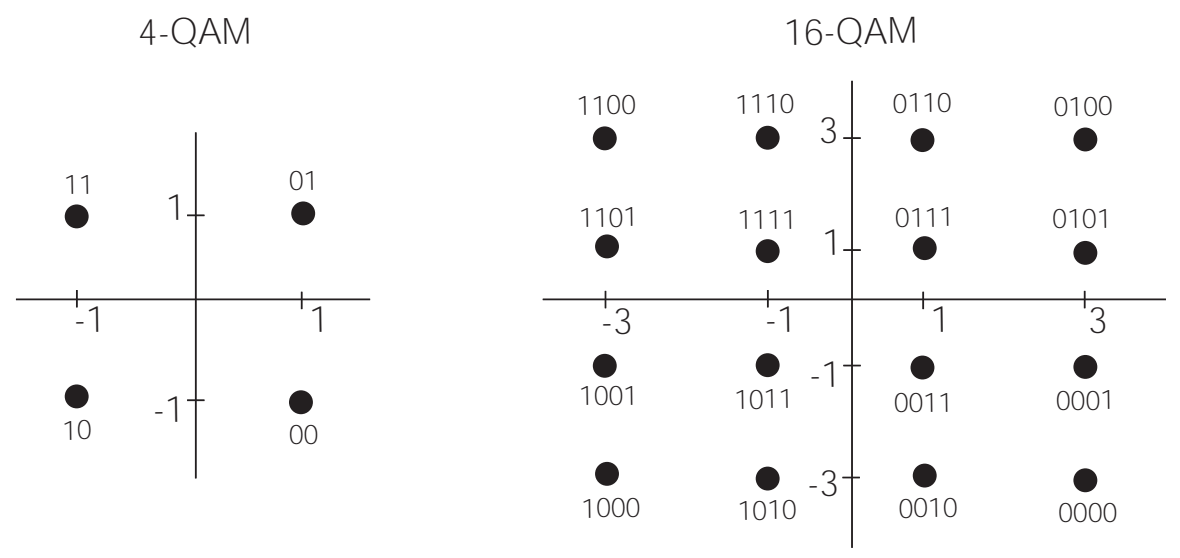

Figure 1.4: The 4-QAM and 16-QAM constellations.

\subsection{Multiple-Input Multiple-Output Channels}

If the channel is in a deep fade, i.e. if $|h|$ is small, then the error rate will be high. By introducing multiple transmit and/or receive antennas, the effects of this scenario can be combated. Intuitively, there is a diversity gain achieved by adding more antennas. Assuming the antennas are far enough apart, the transmitted signal can now travel along multiple independent paths; if one path is in a deep fade, another path can be used to recover the transmitted signal (see Figure 1.5).

\subsubsection{MIMO Channel Model}

Incorporating multiple transmit and receive antennas into the discrete-time baseband model derived above is simple: instead of receiving a single complex number, $r$, each 

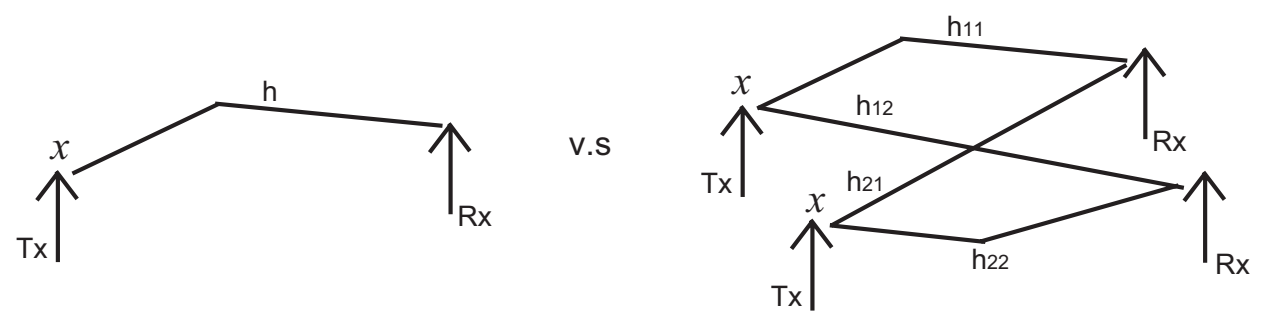

Figure 1.5: By using multiple transmit and receive antennas, $x$ can be sent along multiple paths in the channel to achieve better error performance.

time slot, a matrix, $\mathbf{R}$, of complex numbers is received every $L$ time slots. Specifically,

$$
\mathbf{R}=\mathbf{H C}+\mathbf{N}
$$

where for $k \in \mathcal{I}_{L}$ and $j \in \mathcal{I}_{n_{T}}$ :

$$
\left\{\begin{array}{l}
\mathbf{R}=\left[\begin{array}{llll}
\mathbf{r}_{1} & \mathbf{r}_{2} & \ldots & \mathbf{r}_{L}
\end{array}\right], \mathbf{r}_{k}=\left[\begin{array}{llll}
r_{1 k} & r_{2 k} & \ldots & r_{n_{R} k}
\end{array}\right]^{T}, \\
\mathbf{H}=\left[\begin{array}{llll}
\mathbf{h}_{1} & \mathbf{h}_{2} & \ldots & \mathbf{h}_{n_{T}}
\end{array}\right], \mathbf{h}_{j}=\left[\begin{array}{llll}
h_{1 j} & h_{2 j} & \ldots & h_{n_{R}}
\end{array}\right]^{T}, \\
\mathbf{C}=\left[\begin{array}{llll}
\mathbf{c}_{1} & \mathbf{c}_{2} & \ldots & \mathbf{c}_{L}
\end{array}\right], \mathbf{c}_{k}=\left[\begin{array}{llll}
c_{1 k} & c_{2 k} & \ldots & c_{n_{T} k}
\end{array}\right]^{T}, \\
\mathbf{N}=\left[\begin{array}{llll}
\mathbf{n}_{1} & \mathbf{n}_{2} & \ldots & \mathbf{n}_{L}
\end{array}\right], \mathbf{n}_{k}=\left[\begin{array}{llll}
n_{1 k} n_{2 k} & \ldots & n_{n_{R} k}
\end{array}\right]^{T},
\end{array}\right.
$$

where $n_{T}$ is the number of transmit antennas, $n_{R}$ is the number of receive antennas, and $L$ is the number of time slots. Over the $L$ time slots under consideration, the channel matrix, $\mathbf{H}$, is assumed to be fixed; however, it can change independently in the next $L$ time slots. In this case, the channel is referred to as quasi-static [2]. Also, in general the multiple paths may be spatially correlated. We will assume the channel is spatially white, i.e. the values of $\mathbf{H}$ are uncorrelated (and hence independent if Gaussian random variables).

As an example, consider mapping two groups of information bits to two symbols, $\left\{x_{1}, x_{2}\right\}$, in the underlying constellation. The underlying constellation symbols are 
then sent through the channel using two transmit antennas, two receive antennas, and two time slots. One possible scheme is proposed by Alamouti in [3]:

$$
\begin{aligned}
\mathbf{C} & =\left[\begin{array}{ll}
c_{11} & c_{12} \\
c_{21} & c_{22}
\end{array}\right] \\
& =\left[\begin{array}{ll}
x_{1} & -x_{2}^{*} \\
x_{2} & x_{1}^{*}
\end{array}\right] .
\end{aligned}
$$

The resulting model is:

$$
\left[\begin{array}{ll}
r_{11} & r_{12} \\
r_{21} & r_{22}
\end{array}\right]=\left[\begin{array}{ll}
h_{11} & h_{12} \\
h_{21} & h_{22}
\end{array}\right]\left[\begin{array}{cc}
x_{1} & -x_{2}^{*} \\
x_{2} & x_{1}^{*}
\end{array}\right]+\left[\begin{array}{cc}
n_{11} & n_{12} \\
n_{21} & n_{22}
\end{array}\right] .
$$

\subsubsection{MIMO Maximum-Likelihood Decoding Metric}

Given a matrix $\mathbf{C}$ is transmitted through the channel, how can the decoder make the best guess as to what was sent? We will define the "best guess" as the one that maximizes the a posteriori probability, $P(\mathbf{C} \mid \mathbf{R})$, since this will minimize the probability of errors. The more channel information known by the transmitter and/or receiver, the higher the probability that the best guess will be the correct guess. In this thesis we will assume the CSI is not known by the transmitter (an open loop system), but is known perfectly by the receiver. In actual systems it is unrealistic to assume perfect CSI at the receiver; however, this assumption makes analysis substantially easier, and it is reasonable for systems that employ channel measurement techniques, such as transmitting training sequences.

The best guess the receiver can make is to pick, out of all possible transmission matrices, the $\mathbf{C}$ that maximizes the a posteriori probability:

$$
\hat{\mathbf{C}}=\underset{\text { all } \mathbf{C}}{\operatorname{argmax}}\{P(\mathbf{C} \mid \mathbf{R})\}
$$


Assuming each transmission matrix is equally probable, then using Bayes' rule this is equal to

$$
\hat{\mathbf{C}}=\underset{\text { all } \mathbf{C}}{\operatorname{argmax}}\{P(\mathbf{R} \mid \mathbf{C})\} .
$$

If the CSI is known by the receiver, $P(\mathbf{R} \mid \mathbf{C})$ modifies to the likelihood probability $P(\mathbf{R} \mid \mathbf{C}, \mathbf{H})$. Therefore, the best guess the decoder can make (in the sense of maximizing $P(\mathbf{C} \mid \mathbf{R})$ ) is to maximize the Maximum-Likelihood (ML) metric

$$
\hat{\mathbf{C}}=\underset{\text { all } \mathbf{C}}{\operatorname{argmax}}\{P(\mathbf{R} \mid \mathbf{C}, \mathbf{H})\} .
$$

Assuming the entities of $\mathbf{N}$ are independent circular symmetric complex Gaussian random variables, then $\operatorname{vec}(\mathbf{R}) \sim N_{c}\left(\operatorname{vec}(\mathbf{H C}), \sigma^{2} \mathbf{I}\right)$, i.e., the distribution of $\operatorname{vec}(\mathbf{R})$ is multivariate Gaussian with mean vector $\operatorname{vec}(\mathbf{H C})$ and covariance matrix $\sigma^{2} \mathbf{I}$. Therefore, maximizing $P(\mathbf{R} \mid \mathbf{C}, \mathbf{H})$ reduces to minimizing the argument in the complex exponential, which is the squared Frobenius norm $\|\mathbf{R}-\mathbf{H C}\|^{2}$. Therefore, the ML decoding metric reduces to:

$$
\hat{\mathbf{C}}=\underset{\text { all } \mathbf{C}}{\operatorname{argmin}}\left\{\|\mathbf{R}-\mathbf{H C}\|^{2}\right\} .
$$

In other words, the best guess the decoder can make is to calculate $\|\mathbf{R}-\mathbf{H C}\|^{2}$ for every possible $\mathbf{C}$ that could have been sent, and pick the $\mathbf{C}$ that yields the smallest metric value. This is referred to as an exhaustive search, and its complexity is exponential in $n_{s}$, the number of symbols that have been mapped to $\mathbf{C}$. For example, if $\mathbf{C}$ transmits 2 symbols (e.g. as in (1.2)), and the underlying constellation is 16-QAM, then $\|\mathbf{R}-\mathbf{H C}\|^{2}$ must be calculated $16^{2}=256$ times. When doing an exhaustive search, it is common practice to expand and simplify $(1.3)$ as much as possible to try and reduce this complexity. If $\mathbf{C}$ is designed appropriately, the complexity of an exhaustive search can be reduced to a linear function of $n_{s}$ (see Section 1.4.3). 
If the complexity of an exhaustive search is prohibitive, decoding algorithms can instead be applied that trade-off complexity for error performance; i.e., they no longer are guaranteed to minimize the ML metric, but they are less complex to implement. Decoding algorithms are discussed in detail in Section 1.5 .

\subsubsection{MIMO Encoding Schemes}

The previous section dealt with the decoding metric and provided an algorithm to make the best guess as to what $\mathbf{C}$ was sent given the receiver knows the CSI and the additive noise is white and Gaussian. However, how does one design $\mathbf{C}$ to have good error performance, a high transmission rate, and a reasonable ML decoding complexity?

There are two approaches to improving system performance using multiple transmit antennas:

1. Spatial Multiplexing/Layered MIMO Systems: This approach involves sending independent symbols from each transmit antenna, therefore resulting in a high symbol transmission rate. The most popular scheme is Vertical Bell Labs SpaceTime (V-BLAST) [4]. In general, this higher transmission rate comes at the expense of bit error performance; however, schemes such as Diagonal Bell Labs Space-Time (D-BLAST) attempt to provide a more balanced trade-off at the expense of lower rate and increased decoding complexity. The spatial multiplexing approach is not the focus of the thesis, but more details can be found in [5, chapt. 9].

2. Space-Time Coding: This approach involves sending the same symbol multiple times from different transmit antennas. The redundancy across space and time results in a relatively low symbol transmission rate (compared to V-BLAST), but the 
error performance is much improved. This thesis will focus on the space-time coding approach. Specifically, the focus will be on an encoding scheme referred to as linear space-time block coding.

Space-time block coding involves sending a group of symbols repeatedly over space (via multiple transmit antennas) and over time (via multiple time slots). For example, a simple space-time block code (STBC) is the Alamouti code (1.2). This code uses two transmit antennas to send two symbols over two time slots, therefore resulting in an average symbol rate of one symbol per time slot due to the redundancy embedded in the transmission matrix. A STBC is not limited to sending one symbol per transmit antenna per time slot. In general, a group of symbols added or multiplied together may be sent from each transmit antenna per time slot. However, we will focus on linear STBCs. These are STBCs in which $n_{s}$ symbols are mapped to a matrix $\mathbf{C}$, which can be represented using linear dispersion matrices [6] as follows:

$$
\mathbf{C}=\sum_{k=1}^{n_{s}}\left(\Re\left\{x_{k}\right\} \mathbf{A}_{k}+j \Im\left\{x_{k}\right\} \mathbf{B}_{k}\right)
$$

where each $\mathbf{A}_{k}$ and $\mathbf{B}_{k}$ is a fixed (in general complex-valued) matrix of dimension $n_{T} \times L$. For example, the Alamouti code $(1.2)$ is a linear STBC and can be represented using the following linear dispersion matrices:

$$
A_{1}=\left[\begin{array}{ll}
1 & 0 \\
0 & 1
\end{array}\right], A_{2}=\left[\begin{array}{cc}
0 & -1 \\
1 & 0
\end{array}\right], B_{1}=\left[\begin{array}{cc}
1 & 0 \\
0 & -1
\end{array}\right], B_{2}=\left[\begin{array}{ll}
0 & 1 \\
1 & 0
\end{array}\right] .
$$

When designing linear STBCs, there is a trade-off among good error performance, high transmission rate, and low ML decoding complexity. Let's consider the error performance and decoding complexity in detail. 


\section{Error Performance}

Through probability analysis, one can either determine exactly or estimate accurately at high signal-to-noise ratios (SNRs) the average error probability for common linear STBCs in popular fading channel models. In either case, the error expressions have the following form:

$$
P(\text { error }) \propto\left(G_{c}\right)^{-G_{d}}(S N R)^{-G_{d}},
$$

where $G_{c}$ is referred to as the coding gain and $G_{d}$ is referred to as the diversity gain. If the error rate versus SNR is plotted on a log-log scale, the diversity gain can be interpreted as the slope of the curve at high SNR; the coding gain corresponds to the horizontal position of the curve. From (1.5) it is clear that maximizing the diversity gain is more important at high SNR. In fact, the diversity gain is an important measure and can be defined more formally as follows [7]:

$$
G_{d}=-\lim _{\mathrm{SNR} \rightarrow \infty} \frac{\log P \text { (error) }}{\log \mathrm{SNR}} .
$$

For a linear STBC with $n_{T}$ transmit antennas and $n_{R}$ receive antennas, the maximum diversity gain achievable is equal to $n_{T} \times n_{R}$ [8]. A STBC/decoder pair that achieves this is referred to as being "full-diversity."

\section{Decoding Complexity}

As discussed in Section 1.4.2, the ML decoding complexity is generally exponential in $n_{s}$. However, depending on how $\mathbf{C}$ is designed, the expanded and simplified squared Frobenius norm expression, $\|\mathbf{R}-\mathbf{H C}\|^{2}$, may result in the transmitted symbols being partially or completely decoupled from each other. If the symbols are completely decoupled, the ML decoding complexity reduces to a linear function of $n_{s}$. A STBC with such a property is referred to as being orthogonal. 
Linear STBCs that are orthogonal are desirable because of this complete decoupling in the expanded metric expression. An example of a linear orthogonal space-time block code (OSTBC) is the Alamouti code (1.2). In [9] a whole family of OSTBCs are constructed based on generalized complex orthogonal designs.

OSTBCs are desirable, but if the underlying constellation is complex, rate-one full-diversity OSTBCs do not exist for more than two transmit antennas. Therefore, for systems with more than two transmit antennas, either diversity gain or symbol rate must be sacrificed to achieve orthogonality. For example, the following four transmit antenna STBC achieves orthogonality and full-diversity, but it only has a rate of $3 / 4[5$, chapt. 4$]$ :

$$
\begin{aligned}
\mathbf{C}= & {\left[\begin{array}{cccc}
c_{11} & c_{12} & c_{13} & c_{14} \\
c_{21} & c_{22} & c_{23} & c_{24} \\
c_{31} & c_{32} & c_{33} & c_{34} \\
c_{41} & c_{42} & c_{43} & c_{44}
\end{array}\right] } \\
= & {\left[\begin{array}{cccc}
x_{1} & -x_{2}^{*} & x_{3}^{*} & 0 \\
x_{2} & x_{1}^{*} & 0 & x_{3}^{*} \\
x_{3} & 0 & -x_{1}^{*} & -x_{2}^{*} \\
0 & x_{3} & x_{2} & -x_{1}
\end{array}\right] . }
\end{aligned}
$$

Quasi-orthogonal STBCs (QOSTBCs) [10-12] tradeoff decoding complexity for better error and rate performance. For example, consider the four-transmit antenna QOSTBC proposed in [10]: 


$$
\begin{aligned}
\mathbf{C}= & {\left[\begin{array}{llll}
c_{11} & c_{12} & c_{13} & c_{14} \\
c_{21} & c_{22} & c_{23} & c_{24} \\
c_{31} & c_{32} & c_{33} & c_{34} \\
c_{41} & c_{42} & c_{43} & c_{44}
\end{array}\right] } \\
= & {\left[\begin{array}{cccc}
x_{1} & -x_{2}^{*} & -x_{3}^{*} & x_{4} \\
x_{2} & x_{1}^{*} & -x_{4}^{*} & -x_{3} \\
x_{3} & -x_{4}^{*} & x_{1}^{*} & -x_{2} \\
x_{4} & x_{3}^{*} & x_{2}^{*} & x_{1}
\end{array}\right] . }
\end{aligned}
$$

This STBC can be made full diversity using a technique referred to as constellation rotation $[13,14]$. Additionally, this STBC has a rate of one. However, the expanded ML metric expression is no longer completely decoupled; it can only be partially decoupled into two independent functions $f_{14}\left(x_{1}, x_{4}\right)+f_{23}\left(x_{2}, x_{3}\right)$.

\subsubsection{Vectorization of Channel Model}

As derived in Section 1.4.1, the discrete-time baseband channel model (1.1) is expressed in terms of matrices with complex entities. The resulting ML decoding metric for this model is expressed in $(1.3)$. In general, 11.3 is the squared Frobenius norm of a complex matrix. When implementing and designing decoding algorithms, it is often more convenient to express 1.1 in terms of an equivalent real channel model that involves vectors and matrices with real entities. This has the additional benefit of resulting in an ML metric that is expressed in terms of the squared Euclidean norm of a real vector. 
Assume $\mathbf{C}$ is a linear STBC that transmits $n_{s}$ symbols from the underlying constellation. $\mathbf{C}$ can therefore be expressed in terms of its linear dispersion matrices (1.4). The standard way of vectorizing the channel model uses the linear dispersion matrices and is outlined in [8, pp. 97-99]:

$$
\mathbf{r}^{\prime}=\mathbf{F}^{\prime} \mathbf{x}^{\prime}+\mathbf{n}^{\prime}
$$

where

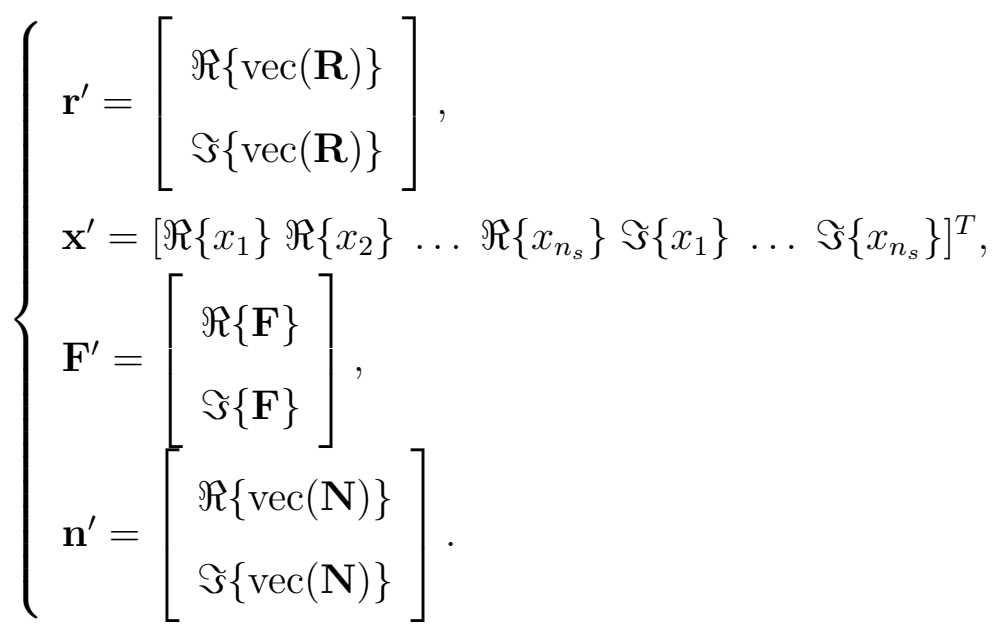

$\mathbf{F}$ is defined using the linear dispersion matrices such that $\mathbf{F} \mathbf{x}^{\prime}=\operatorname{vec}(\mathbf{H C})$. In this vectorization $\mathbf{r}^{\prime} \in \mathbb{R}^{2 n_{R} L \times 1}, \mathbf{F}^{\prime} \in \mathbb{R}^{2 n_{R} L \times 2 n_{s}}$, and $\mathbf{x}^{\prime} \in \mathbb{R}^{2 n_{s} \times 1}$. In this representation any redundancy in the transmission matrix, $\mathbf{C}$, has been removed and shifted into $\mathbf{F}^{\prime}$. This results in the following ML metric:

$$
\hat{\mathbf{x}}^{\prime}=\underset{x^{\prime} \in S^{n_{s}}}{\operatorname{argmin}}\left\{\left\|\mathbf{r}^{\prime}-\mathbf{F}^{\prime} \mathbf{x}^{\prime}\right\|^{2}\right\} .
$$

Note that it is straightforward to show that (1.7) is equal to (1.3).

Applying this standard vectorization to the system model has a number of advantages: 1) It results in real matrices and vectors, which suit matrix manipulations often implemented in decoding algorithms (like QR factorization or pseudo-inverse). 2) It shifts the redundancy from $\mathbf{C}$ into $\mathbf{F}^{\prime}$. Working with the vector $\mathbf{x}^{\prime}$ directly suits 
many decoding algorithms (like zero-forcing and sphere decoding). 3) The resulting ML metric is a squared Euclidean norm, which makes it easier to expand and simplify the metric expression.

\subsection{MIMO Decoding Algorithms}

As discussed in Section 1.4.2, the best guess the decoder can make (in the ML sense) is to evaluate $1.3 \mathrm{p}$. An algorithm that achieves this is referred to as optimal, and a simple example of such an algorithm is an exhaustive search. Alternatively, one could implement a sub-optimal algorithm: one that trades off error performance for a reduction in decoding complexity.

\subsubsection{Sub-optimal Decoding Algorithms}

There are many sub-optimal decoding algorithms that offer varying levels of trade-off between complexity and error performance. Sub-optimal algorithms are primarily used in layered MIMO systems (see Section 1.4.3) since in these systems the metric cannot be decoupled, which makes an exhaustive search prohibitive. The most popular sub-optimal decoding algorithms can be broadly categorized as follows:

\section{The Unconstrained Least-Squares Approach}

Using the vectorized system model, the ML solution is the one that minimizes the metric $\left\|\mathbf{r}^{\prime}-\mathbf{F}^{\prime} \mathbf{x}^{\prime}\right\|^{2}$. Minimizing this metric is referred to as solving the integer leastsquares problem [15]. If we were to assume $\mathbf{x}^{\prime}$ could take on any real value, the solution would simply be $\mathbf{x}^{\prime}=\mathbf{F}^{\dagger} \mathbf{r}^{\prime}$, where $\mathbf{F}^{\dagger}$ is the pseudo-inverse of $\mathbf{F}^{\prime}$. This amounts to 
solving the unconstrained least-squares problem; however, the ML expression is a constrained least-squares problem: $\mathbf{x}^{\prime}$ cannot take on any real value, but instead a finite set of integer values.

One approach to developing sub-optimal algorithms is to evaluate $\mathbf{x}^{\prime}=\mathbf{G r}^{\prime}$, and then slice $\mathbf{x}^{\prime}$ to the nearest valid constellation point. If $\mathbf{G}=\mathbf{F}^{\dagger}$, the solution is referred to as "zero-forcing." But $\mathbf{G}$ need not be the pseudo-inverse. For example, a sub-optimal algorithm that outperforms zero-forcing is minimum mean square error (MMSE) detection. This algorithm is based on minimizing the expected value of the mean square error between the transmitted vector and its estimate. The algorithm for MMSE detection is the same as for zero-forcing, except that the filter $\mathbf{G}$ is calculated differently.

\section{Nulling and Cancelling}

In this approach, the solution $\mathbf{x}^{\prime}=\mathbf{G r}^{\prime}$ is evaluated as above, but instead of slicing all values of $\mathbf{x}^{\prime}$ to the nearest constellation point, only one of the coordinates is sliced. This coordinate value is then assumed to be correct, and its contribution is subtracted from the received signal to obtain a reduced-order integer least-squares problem. The process is then repeated.

This algorithm is more complex to implement, but offers better error performance than simply slicing all coordinates of $\mathbf{x}^{\prime}$ at the same time. However, it can suffer from error-propagation if a coordinate if estimated incorrectly. Therefore, it is beneficial to employ "optimal ordering" by performing nulling and cancelling from the strongest to the weakest signal. This is the sub-optimal decoding method employed in V-BLAST and is referred to as V-BLAST detection [4]. 


\section{Lattice Reduction}

The performance of finding the unconstrained least-squares solution and then slicing depends on how orthogonal the columns of $\mathbf{F}^{\prime}$ are. For example, if all the columns of $\mathbf{F}^{\prime}$ are completely orthogonal (e.g., when an OSTBC is being transmitted), then the zero-forcing solution will be the ML solution. This is because zero-forcing effectively decodes each symbol by itself, ignoring the interference or coupling from the other symbols; if $\mathbf{F}^{\prime}$ is orthogonal (column-wise) the metric will have no coupled terms, and therefore there is no loss associated with ignoring the interference.

Therefore, it is advantageous to try and manipulate $\mathbf{F}^{\prime}$ in a way that makes the columns more orthogonal. This approach is referred to as lattice reduction. The process of lattice reduction itself has an exponential complexity; however, sub-optimal algorithms such as the LLL algorithm [16] can be employed.

\subsubsection{The Sphere Decoding Algorithm}

If an optimal decoding algorithm is required, one popular candidate is the Sphere Decoding Algorithm (SDA) [17-19]. This algorithm is equivalent to an exhaustive search, yet it has been shown to be efficient over a large range of SNRs, despite the expected complexity growing exponentially with $n_{s}[20]$. In fact, in many practical cases, the algorithm behaves like one of polynomial complexity over the SNR window of interest [15].

The SDA was originally proposed in [21] to solve the closest point problem in a lattice with no exploitable structure. The premise is simple: search only the valid lattice points that lie inside a hypersphere of radius $r$ around the point under consideration, $x$ (see Figure 1.6). The novelty of the SDA is in the method by which the 


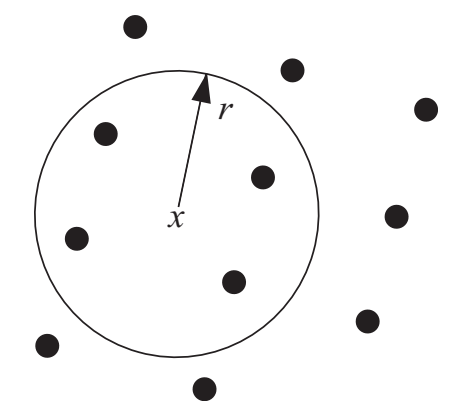

Figure 1.6: Geometric interpretation of the SDA.

points inside the hypersphere are found. Specifically, the lattice generator matrix is decomposed using a $\mathrm{QR}$ decomposition, and then the upper triangle matrix, $\mathbf{R}$, is used to eliminate, dimension by dimension, all co-ordinates that cannot lie inside the hypersphere.

The SDA is applied to the MIMO decoding problem by performing the standard vectorization of the system model outlined in Section 1.4.4. The effective real channel matrix, $\mathbf{F}^{\prime}$, can then be interpreted as a lattice generating matrix, and ML decoding becomes equivalent to solving the closest point problem in the lattice generated by $\mathbf{F}^{\prime}$.

\section{Overview of Basic SDA}

A review of the SDA is in order since it is used in simulations later in the thesis.

Assume we have performed a vectorization of the system model and we want to solve the MIMO detection problem (1.7). We will assume $\mathbf{F}^{\prime}$ is an $n \times m$ matrix where $n \geq m$. A radius $C$ is selected, and the SDA is applied to find all points that lie inside the hypersphere of radius $C$ around the received vector $\mathbf{r}^{\prime}$. The lattice has been skewed by the generator matrix $\mathbf{F}^{\prime}$. Therefore, we want to find every point 
$\mathbf{x}^{\prime}=\left(x_{1}^{\prime}, x^{\prime}{ }_{2}, \ldots, x_{m}^{\prime}\right)$ that satisfies

$$
C^{2} \geq\left\|\mathbf{r}^{\prime}-\mathbf{F}^{\prime} \mathbf{x}^{\prime}\right\|^{2} .
$$

The basis of the algorithm is as follows: by expressing $\mathbf{F}^{\prime}$ in terms of its QR decomposition, (1.8) can be equivalently expressed as follows:

$$
\begin{aligned}
C^{2} \geq\left\|\mathbf{r}^{\prime}-\mathbf{F}^{\prime} \mathbf{x}^{\prime}\right\|^{2} & =\left\|\mathbf{r}^{\prime}-\mathbf{Q R} \mathbf{x}^{\prime}\right\|^{2} \\
& =\left\|\mathbf{r}^{\prime}-\left[\begin{array}{l}
\mathbf{Q}_{1} \\
\mathbf{Q}_{2}
\end{array}\right]\left[\begin{array}{c}
\mathbf{R} \\
\mathbf{0}
\end{array}\right] \mathbf{x}^{\prime}\right\|^{2} \\
& =\left\|\left[\begin{array}{l}
\mathbf{Q}_{1}^{T} \\
\mathbf{Q}_{2}^{T}
\end{array}\right] \mathbf{r}^{\prime}-\left[\begin{array}{c}
\mathbf{R} \\
\mathbf{0}
\end{array}\right] \mathbf{x}^{\prime}\right\|^{2} \\
& =\left\|\mathbf{Q}_{1}^{T} \mathbf{r}^{\prime}-\mathbf{R} \mathbf{x}^{\prime}\right\|^{2}+\left\|\mathbf{Q}_{2}^{T} \mathbf{r}^{\prime}\right\|^{2}
\end{aligned}
$$

where $\mathbf{Q}_{1}$ and $\mathbf{Q}_{2}$ are the first $m$ and $n-m$ columns of $\mathbf{Q}$. Note that $\mathbf{Q} \mathbf{Q}^{T}=\mathbf{I}$. The above expression can now be re-written as

$$
C^{\prime 2} \geq\left\|\mathbf{y}-\mathbf{R x}^{\prime}\right\|^{2}
$$

where $C^{\prime 2}=C^{2}-\left\|\mathbf{Q}_{2}^{T} \mathbf{r}^{\prime}\right\|^{2}$ and $\mathbf{y}=\mathbf{Q}_{1}^{T} \mathbf{r}^{\prime}$. The term $\left\|\mathbf{y}-\mathbf{R} \mathbf{x}^{\prime}\right\|^{2}$ can be expanded as follows:

$$
\begin{aligned}
\left\|\mathbf{y}-\mathbf{R} \mathbf{x}^{\prime}\right\|^{2} & =\sum_{i=1}^{m}\left(y_{i}-\sum_{j=1}^{m} r_{i j} x_{j}^{\prime}\right)^{2} \\
& =\left(y_{m}-r_{m, m} x^{\prime}{ }_{m}\right)^{2}+\left(y_{m-1}-r_{m-1, m} x^{\prime}{ }_{m}-r_{m-1, m-1} x^{\prime}{ }_{m-1}\right)^{2}+\ldots
\end{aligned}
$$

Therefore, a necessary (but not sufficient) condition for $\mathbf{x}^{\prime}$ to be in the hypersphere is

$$
C^{\prime 2} \geq\left(y_{m}-r_{m, m} x_{m}^{\prime}\right)^{2},
$$


which implies

$$
\left\lceil\frac{-C^{\prime}+y_{m}}{r_{m, m}}\right\rceil \leq x_{m}^{\prime} \leq\left\lfloor\frac{C^{\prime}+y_{m}}{r_{m, m}}\right\rfloor .
$$

Given a coordinate $x_{m}^{\prime}$ that satisfies 1.10 , the set of coordinates $\left\{x^{\prime}{ }_{m-1}\right\}$ that satisfy

$$
C^{2} \geq\left(y_{m}-r_{m, m} x_{m}^{\prime}\right)^{2}+\left(y_{m-1}-r_{m-1, m} x_{m}^{\prime}-r_{m-1, m-1} x_{m-1}^{\prime}\right)^{2}
$$

are then determined.

The algorithm continues in this fashion, working its way down to the coordinate $x^{\prime}{ }_{1}$, effectively "chopping away" sets of coordinate values (points) not in the hypersphere. The algorithm can be formalized as follows [15]:

Initialization: Set $k=m$, calculate $C^{2}$ and $\mathbf{y}$, let $y_{m \mid m+1}=y_{m}$ and $C_{m}^{\prime 2}=C^{\prime 2}$.

1. (Bound for $x^{\prime}{ }_{k}$ ) Set $\mathrm{UB}\left(x^{\prime}{ }_{k}\right)=\left\lfloor\frac{C^{\prime}{ }_{k}+y_{k \mid k+1}}{r_{k, k}}\right\rfloor, x^{\prime}{ }_{k}=\left\lceil\frac{-C^{\prime}{ }_{k}+y_{k \mid k+1}}{r_{k, k}}\right\rceil-1$.

2. (Increase $\left.x^{\prime}{ }_{k}\right) x^{\prime}{ }_{k}=x^{\prime}{ }_{k}+1$. If $x^{\prime}{ }_{k} \leq \mathrm{UB}\left(x^{\prime}{ }_{k}\right)$, go to 4 ; else, go to 3 .

3. (Increase $k$ ) $k=k+1$; if $k=m+1$, terminate algorithm; else go to 2 .

4. (Decrease $k$ ) If $k=1$, go to 5 ; else $k=k-1, y_{k \mid k+1}=y_{k}-\sum_{j=k+1}^{m} r_{k, j} x^{\prime}$, $C^{\prime 2}=C^{\prime 2}{ }_{k+1}-\left(y_{k+1 \mid k+2}-r_{k+1, k+1} x^{\prime}{ }_{k+1}\right)^{2}$, and go to 1 .

5. Solution found. Save $\mathbf{x}^{\prime}$ and its metric; go to 2 .

Once all points inside the hypersphere are enumerated, the point with the smallest corresponding metric value will be the most-likely candidate. A detailed examination of the SDA, along with a complexity analysis can be found in $[15,17,20]$ and references therein. 


\section{Improvements to the Basic SDA}

The SDA explained in the above section is the basic Fincke and Pohst implementation [21]. In the improved Viterbo and Boutros implementation [19], the radius is changed adaptively during the search. Specifically, the radius is updated (made smaller) each time a point is found, and the algorithm is re-started. In the improved Schnorr-Euchner implementation of the SDA [22], the interval of possible coordinates inside the sphere are spanned in a zig-zag order, starting from the midpoint of the interval. In [23] it is concluded, based on numerical results, that the Schnorr-Euchner implementation is more efficient than the Viterbo-Boutros implementation. Additionally, the Schnorr-Euchner implementation is less sensitive to initial radius selection. Initial radius selection is a separate problem on its own, and yet the initial radius is crucial to the performance of the SDA. If the radius is too large, the search remains exponential in size; if the radius is too small, there will be no points inside the hypersphere, and the algorithm will have to be restarted with a larger radius. In the latter case, by what amount should one increase the radius? Currently, there are two approaches to initial radius selection. One approach is to set the initial radius based on a simple sub-optimal guess (e.g. zero-forcing) [15]. The second approach is to pick

the initial radius based on the smallest eigenvalue in the Gram matrix $\mathbf{G}=\mathbf{F}^{\prime T} \mathbf{F}^{\prime}[18]$.

Other improvements to the SDA are dicussed in [24-28]. This thesis is concerned with the SDA applied to QOSTBCs, and therefore, [25] is particularly of interest. In [25] it is shown that the quasi-orthogonal property can be used to write the ML metric as two independent Euclidean norms. The SDA can then be applied independently and in parallel to both norms to reduce the overall complexity. 


\subsection{Conclusion}

The above introductory material served as a review of the relevant concepts needed to understand the contribution of the thesis. The remainder of the thesis is organized as follows. In chapter 2 a new vectorization for a linear space-time block code will be introduced that results in a new generic ML metric expression. The new metric expression allows one to quickly check the degree of decoupling in the metric for a given space-time block code, and as such can assist in designing new space-time block codes. A new QOSTBC with low-decoding complexity is constructed as an example. In chapter 3 the optimal and sub-optimal decoding of QOSTBCs is then considered in the context of the new ML metric expression. Chapter 4 then discusses future work, with an emphasis on two of the open problems resulting from the contributions in chapter 2 and chapter 3. 


\section{Chapter 2}

\section{Alternative Vectorization for a Linear STBC}

\section{$2.1 \quad$ Introduction}

In this chapter, a new vectorization for a linear STBC is proposed that explicitly maintains the redundancy in the STBC matrix. By expressing the ML metric using the new vectorization, a new generic representation of the ML metric expression for any linear STBC is derived. One immediate application of this new metric expression is the convenient partial decoupling and simplification of the metric for linear STBCs.

The new metric expression can also be used as a design tool to help in the construction of new STBCs with low decoding complexity. As an example, a new QOSTBC is constructed with lower decoding complexity than that previously proposed in the literature. 


\subsection{An Alternative Vectorization}

Consider a system with $n_{T}$ transmit and $n_{R}$ receive antennas. $n_{s}$ symbols $\left\{x_{1}, x_{2}, \ldots, x_{n_{s}}\right\}$ from the underlying constellation(s) $\left\{S_{i}\right\}_{i=1}^{n_{s}}$ are mapped to a linear STBC, C, of duration $L$ time slots. The constellations are arbitrary and may or may not be rotated. Assume the channel is frequency flat and spatially white. Furthermore, assume the channel is time-invariant over the $L$ time slots and changes independently in the following $L$ slots, i.e., it is quasi-static. Using the equivalent discrete-time baseband model, the received matrix can be expressed as in 1.1). The elements of $\mathbf{H}$ are drawn from an arbitrary distribution, but are assumed to be perfectly known to the receiver (perfect channel state information, CSI). The elements of $\mathbf{N}$ are assumed to be independent identically distributed (circular symmetric) complex Gaussian random variables. Therefore, the decoder chooses the codeword matrix, $\mathbf{C}$, that minimizes the metric

$$
M=\|\mathbf{R}-\mathbf{H C}\|^{2} .
$$

An equivalent representation of the metric is derived using the standard vectorization in Section 1.4.4:

$$
M=\left\|\mathbf{r}^{\prime}-\mathbf{F}^{\prime} \mathbf{x}^{\prime}\right\|^{2} .
$$

An alternative vectorization can be proposed that results in the same expanded ML metric expression, but is written in a form that maintains the redundancy in $\mathbf{C}$ instead of $\mathbf{F}^{\prime}$. This alternative vectorization is proposed below and is used in subsequent proofs and analyses.

Let

$$
M=\left\|\mathbf{y}^{\prime}-\mathbf{G}^{\prime} \mathbf{c}^{\prime}\right\|^{2}
$$


where

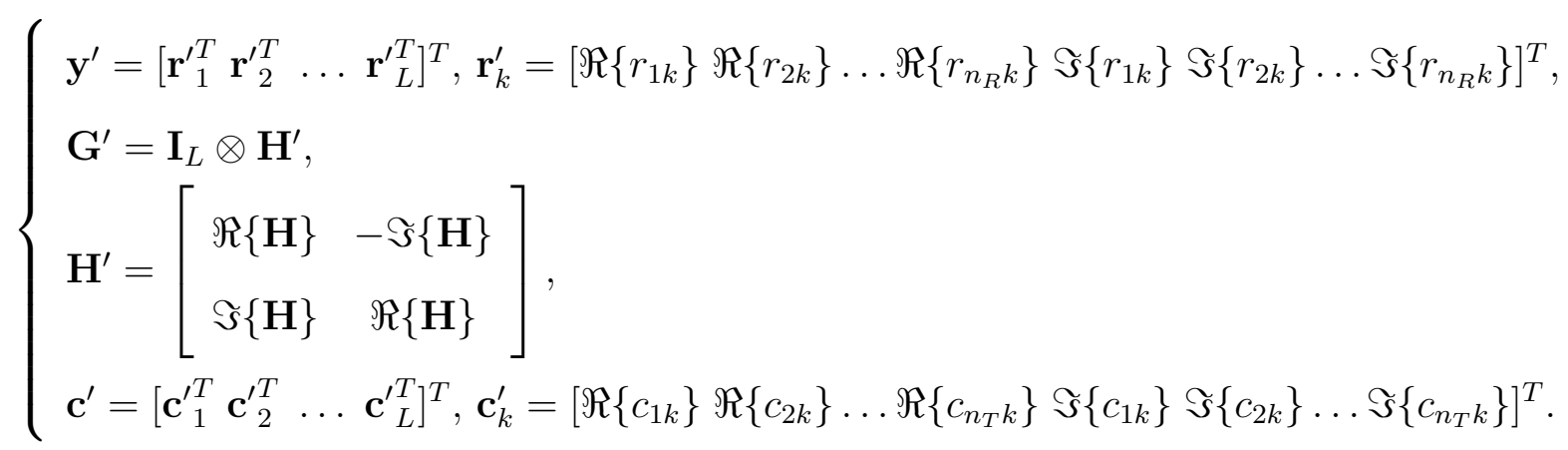

Note that in this vectorization $\mathbf{G}^{\prime}$ is a $2 n_{R} L \times 2 n_{T} L$ block-diagonal matrix. $\mathbf{y}^{\prime}$ is a $2 n_{R} L \times 1$ real vector (a permuted version of $\mathbf{r}^{\prime}$ in the standard vectorization), and $\mathbf{c}^{\prime}$ is a $2 n_{T} L \times 1$ real vector (the transmission matrix vectorized).

Lemma 1 The ML metric expression using the new vectorization (2.3) is equal to the $M L$ metric expression using the standard vectorization (2.2):

$$
\left\|\mathbf{r}^{\prime}-\mathbf{F}^{\prime} \mathbf{x}^{\prime}\right\|^{2}=\left\|\mathbf{y}^{\prime}-\mathbf{G}^{\prime} \mathbf{c}^{\prime}\right\|^{2} .
$$

Moreover, each term of the expanded metric is equal:

$$
\left\|\mathbf{r}^{\prime}\right\|^{2}+\left\|\mathbf{F}^{\prime} \mathbf{x}^{\prime}\right\|^{2}-2 \mathbf{r}^{\prime T} \mathbf{F}^{\prime} \mathbf{x}^{\prime}=\left\|\mathbf{y}^{\prime}\right\|^{2}+\left\|\mathbf{G}^{\prime} \mathbf{c}^{\prime}\right\|^{2}-2 \mathbf{y}^{\prime T} \mathbf{G}^{\prime} \mathbf{c}^{\prime}
$$

where $\left\|\mathbf{r}^{\prime}\right\|^{2}=\left\|\mathbf{y}^{\prime}\right\|^{2},\left\|\mathbf{F}^{\prime} \mathbf{x}^{\prime}\right\|^{2}=\left\|\mathbf{G}^{\prime} \mathbf{c}^{\prime}\right\|^{2}=\|\mathbf{H C}\|^{2}$, and $\mathbf{r}^{\prime T} \mathbf{F}^{\prime} \mathbf{x}^{\prime}=\mathbf{y}^{\prime T} \mathbf{G}^{\prime} \mathbf{c}^{\prime}$.

Proof: See the Appendix.

\subsection{Expanded ML Metric}

Using the new vectorization, the expanded ML metric is derived in the following lemma. 
Lemma 2 The most-likely transmission matrix, $\mathbf{C}$, is the one that minimizes the following metric: $M(\mathbf{C})$

$$
\begin{aligned}
= & \underbrace{}_{\text {coupled symbols }} \underbrace{n_{T} \sum_{m=j+1}^{n_{T}}\left(\Re\left\{\left\langle\mathbf{h}_{j}, \mathbf{h}_{m}\right\rangle\right\} \Re\left\{\left\langle\mathbf{c}^{(m)}, \mathbf{c}^{(j)}\right\rangle\right\}+\Im\left\{\left\langle\mathbf{h}_{j}, \mathbf{h}_{m}\right\rangle\right\} \Im\left\{\left\langle\mathbf{c}^{(m)}, \mathbf{c}^{(j)}\right\rangle\right\}\right)}_{j=1} \\
& +\underbrace{\sum_{j=1}^{n_{T}}\left\|\mathbf{h}_{j}\right\|^{2}\left\|\mathbf{c}^{(j)}\right\|^{2}}_{\text {received energy }}-\underbrace{2 \sum_{k=1}^{L} \sum_{j=1}^{n_{T}}\left(\Re\left\{\left\langle\mathbf{r}_{k}, \mathbf{h}_{j}\right\rangle\right\} \Re\left\{c_{j k}\right\}+\Im\left\{\left\langle\mathbf{r}_{k}, \mathbf{h}_{j}\right\rangle\right\} \Im\left\{c_{j k}\right\}\right)}_{\text {decoupled symbols }} .
\end{aligned}
$$

Proof: See the Appendix.

The above metric expression is valid for any linear STBC. However, if the STBC is constructed using orthogonal or quasi-orthogonal designs (e.g. [9-12]), then its intuitive interpretation becomes very elegant. In this case, the first term in the metric exclusively generates all the coupled terms. Naturally, it reduces to zero if the STBC is orthogonal. The energy term simplifies to $\sum_{j=1}^{n_{T}}\left\|\mathbf{h}_{j}\right\|^{2}\left\|\mathbf{c}^{(j)}\right\|^{2}=\|\left.\mathbf{H}\right|^{2} \sum_{i=1}^{n_{s}}\left|x_{i}\right|^{2}$, and can be eliminated from the metric expression if the underlying constellation is equi-energy. The third term exclusively generates all the decoupled terms.

It is worth noting that for any linear STBC, the metric will always include a term consisting of each decoupled symbol by itself, multiplied or attenuated by a sum of inner products. Specifically, the decoupled symbols in (2.4) can be simplified as follows:

$$
-2 \sum_{k=1}^{L} \sum_{j=1}^{n_{T}}\left(\Re\left\{\left\langle\mathbf{r}_{k}, \mathbf{h}_{j}\right\rangle\right\} \Re\left\{c_{j k}\right\}+\Im\left\{\left\langle\mathbf{r}_{k}, \mathbf{h}_{j}\right\rangle\right\} \Im\left\{c_{j k}\right\}\right)=\sum_{i=1}^{n_{s}}\left(\alpha_{R i} \Re\left\{x_{i}\right\}+\alpha_{I i} \Im\left\{x_{i}\right\}\right)
$$

where $\alpha_{R i}$ and $\alpha_{I i}$ represent a sum of inner products specific to the transmission matrix $\mathbf{C}$. 
A natural question to ask is how do the $\left\{\left\langle\mathbf{r}_{k}, \mathbf{h}_{j}\right\rangle\right\}$ values that precede the decoupled terms in 2.4 contribute to the metric in comparison to the $\left\{\left\langle\mathbf{h}_{j}, \mathbf{h}_{m}\right\rangle\right\}$ values that precede the coupled terms. It turns out that these inner products can be interpreted as reliabilities, which has applications in the development of sub-optimal decoding algorithms and codeword tests. This will be discussed in detail later in the thesis.

\subsection{Examples}

It is desirable to simplify and decouple the metric expression of a STBC as much as possible to reduce the detection complexity. The new generic metric expression makes simplification of the metric for linear STBCs straightforward and systematic. This is shown in the following examples.

\subsubsection{A Simple Orthogonal STBC}

Consider the Alamouti STBC $(1.2)$ with $n_{R}=1$. The metric can be calculated as follows: 


$$
\begin{aligned}
M(\mathbf{C})= & \|\mathbf{H}\|^{2}\left(\left|x_{1}\right|^{2}+\left|x_{2}\right|^{2}\right)-2 \sum_{k=1}^{L} \sum_{j=1}^{n_{T}}\left(\Re\left\{\left\langle\mathbf{r}_{k}, \mathbf{h}_{j}\right\rangle\right\} \Re\left\{c_{j k}\right\}+\Im\left\{\left\langle\mathbf{r}_{k}, \mathbf{h}_{j}\right\rangle\right\} \Im\left\{c_{j k}\right\}\right) \\
= & \|\mathbf{H}\|^{2}\left(\Re\left\{x_{1}\right\}^{2}+\Im\left\{x_{1}\right\}^{2}+\Re\left\{x_{2}\right\}^{2}+\Im\left\{x_{2}\right\}^{2}\right) \\
& -2\left[\Re\left\{x_{1}\right\}\left(\Re\left\{r_{1} h_{1}^{*}\right\}+\Re\left\{r_{2} h_{2}^{*}\right\}\right)+\Im\left\{x_{1}\right\}\left(\Im\left\{r_{1} h_{1}^{*}\right\}-\Im\left\{r_{2} h_{2}^{*}\right\}\right)\right. \\
& \left.+\Re\left\{x_{2}\right\}\left(\Re\left\{r_{1} h_{2}^{*}\right\}-\Re\left\{r_{2} h_{1}^{*}\right\}\right)+\Im\left\{x_{2}\right\}\left(\Im\left\{r_{1} h_{2}^{*}\right\}+\Im\left\{r_{2} h_{1}^{*}\right\}\right)\right] \\
= & \|\mathbf{H} \mid\|^{2} \Re\left\{x_{1}\right\}^{2}+\alpha_{R 1} \Re\left\{x_{1}\right\}+\|\mathbf{H}\|^{2} \Im\left\{x_{1}\right\}^{2}+\alpha_{I 1} \Im\left\{x_{1}\right\} \\
& +\|\mathbf{H}\|^{2} \Re\left\{x_{2}\right\}^{2}+\alpha_{R 2} \Re\left\{x_{2}\right\}+\|\mathbf{H}\|^{2} \Im\left\{x_{2}\right\}^{2}+\alpha_{I 2} \Im\left\{x_{2}\right\}
\end{aligned}
$$

where

$$
\begin{aligned}
& \alpha_{R 1}=-2\left(\Re\left\{r_{1} h_{1}^{*}\right\}+\Re\left\{r_{2} h_{2}^{*}\right\}\right) \\
& \alpha_{I 1}=-2\left(\Im\left\{r_{1} h_{1}^{*}\right\}-\Im\left\{r_{2} h_{2}^{*}\right\}\right) \\
& \alpha_{R 2}=-2\left(\Re\left\{r_{1} h_{2}^{*}\right\}-\Re\left\{r_{2} h_{1}^{*}\right\}\right) \\
& \alpha_{I 2}=-2\left(\Im\left\{r_{1} h_{2}^{*}\right\}+\Im\left\{r_{2} h_{1}^{*}\right\}\right) .
\end{aligned}
$$

As expected, this is equal to the simplified metric expression for the Alamouti scheme derived previously in the literature (e.g. [5, pp. 57]). Note that the $\alpha$ values in front of each symbol consist of a sum of inner products using the received symbols and CSI. For a given coordinate $x_{k}$, its associated $\alpha$ value along with the value of $\|\mathbf{H}\|^{2}$ measures how much $x_{k}$ will contribute to minimizing the metric. Therefore, decoding an OSTBC is simple: first the $\|\mathbf{H}\|^{2}$ and $\alpha$ values are calculated, and then each symbol is independently chosen such that the metric is minimized.

\subsubsection{A QOSTBC with $n_{T}=4$}

Consider the minimum-decoding-complexity quasi-orthogonal STBC (MDC-QOSTBC) 
proposed in $[12$, eqn. (7)]:

$$
\begin{aligned}
\mathbf{C}= & {\left[\begin{array}{llll}
c_{11} & c_{12} & c_{13} & c_{14} \\
c_{21} & c_{22} & c_{23} & c_{24} \\
c_{31} & c_{32} & c_{33} & c_{34} \\
c_{41} & c_{42} & c_{43} & c_{44}
\end{array}\right] } \\
= & {\left[\begin{array}{cccc}
\Re\left\{x_{1}\right\}+j \Re\left\{x_{3}\right\} & -\Re\left\{x_{2}\right\}+j \Re\left\{x_{4}\right\} & -\Im\left\{x_{1}\right\}+j \Im\left\{x_{3}\right\} & \Im\left\{x_{2}\right\}+j \Im\left\{x_{4}\right\} \\
\Re\left\{x_{2}\right\}+j \Re\left\{x_{4}\right\} & \Re\left\{x_{1}\right\}-j \Re\left\{x_{3}\right\} & -\Im\left\{x_{2}\right\}+j \Im\left\{x_{4}\right\} & -\Im\left\{x_{1}\right\}-j \Im\left\{x_{3}\right\} \\
-\Im\left\{x_{1}\right\}+j \Im\left\{x_{3}\right\} & \Im\left\{x_{2}\right\}+j \Im\left\{x_{4}\right\} & \Re\left\{x_{1}\right\}+j \Re\left\{x_{3}\right\} & -\Re\left\{x_{2}\right\}+j \Re\left\{x_{4}\right\} \\
-\Im\left\{x_{2}\right\}+j \Im\left\{x_{4}\right\} & -\Im\left\{x_{1}\right\}-j \Im\left\{x_{3}\right\} & \Re\left\{x_{2}\right\}+j \Re\left\{x_{4}\right\} & \Re\left\{x_{1}\right\}-j \Re\left\{x_{3}\right\}
\end{array}\right] . }
\end{aligned}
$$

First the coupled symbols in (2.4) are generated. Using straightforward simplification:

$$
\begin{aligned}
& 2 \sum_{j=1}^{n_{T}} \sum_{m=j+1}^{n_{T}}\left(\Re\left\{\left\langle\mathbf{h}_{j}, \mathbf{h}_{m}\right\rangle\right\} \Re\left\{\left\langle\mathbf{c}^{(m)}, \mathbf{c}^{(j)}\right\rangle\right\}+\Im\left\{\left\langle\mathbf{h}_{j}, \mathbf{h}_{m}\right\rangle\right\} \Im\left\{\left\langle\mathbf{c}^{(m)}, \mathbf{c}^{(j)}\right\rangle\right\}\right) \\
& =\beta\left(\Re\left\{x_{4}\right\} \Im\left\{x_{4}\right\}+\Im\left\{x_{3}\right\} \Re\left\{x_{3}\right\}-\Re\left\{x_{2}\right\} \Im\left\{x_{2}\right\}-\Im\left\{x_{1}\right\} \Re\left\{x_{1}\right\}\right)
\end{aligned}
$$

where $\beta=4\left(\Re\left\{\left\langle\mathbf{h}_{1}, \mathbf{h}_{3}\right\rangle\right\}+\Re\left\{\left\langle\mathbf{h}_{2}, \mathbf{h}_{4}\right\rangle\right\}\right)$. Note that the metric can be split into 4 noninteracting terms. This verifies that the STBC is indeed a MDC-QOSTBC, i.e., the coupling is only one step away from the orthogonal case. Next, the received energy term in 2.4 is generated: $\sum_{j=1}^{n_{T}}\left\|\mathbf{h}_{j}\right\|^{2}\left\|\mathbf{c}^{(j)}\right\|^{2}$ simplifies to $\|\mathbf{H}\|^{2} \sum_{i=1}^{4}\left|x_{i}\right|^{2}$. Finally, the decoupled symbols in 2.4 are generated:

$$
-2 \sum_{k=1}^{L} \sum_{j=1}^{n_{T}}\left(\Re\left\{\left\langle\mathbf{r}_{k}, \mathbf{h}_{j}\right\rangle\right\} \Re\left\{c_{j k}\right\}+\Im\left\{\left\langle\mathbf{r}_{k}, \mathbf{h}_{j}\right\rangle\right\} \Im\left\{c_{j k}\right\}\right)=\sum_{i=1}^{4}\left(\alpha_{R i} \Re\left\{x_{i}\right\}+\alpha_{I i} \Im\left\{x_{i}\right\}\right)
$$

where $\alpha_{R i}$ and $\alpha_{I i}$ are sums of inner products evaluated using C. For example, $\alpha_{R 1}=-2\left(\Re\left\{\left\langle\mathbf{r}_{1}, \mathbf{h}_{1}\right\rangle\right\}+\Re\left\{\left\langle\mathbf{r}_{2}, \mathbf{h}_{2}\right\rangle\right\}+\Re\left\{\left\langle\mathbf{r}_{3}, \mathbf{h}_{3}\right\rangle\right\}+\Re\left\{\left\langle\mathbf{r}_{4}, \mathbf{h}_{4}\right\rangle\right\}\right)$.

It is straightforward to show the metric expression derived above is equal to [12, eqn. (8)], yet it is obtained more easily and systematically. Note that the $\|\mathbf{H}\|^{2}, \beta$, 
and $\alpha$ terms need only be calculated once during the decoding process. They indicate how much the coupled and decoupled terms will contribute to minimizing the metric. In this example, a single $\beta$ value represents the contribution of the coupled terms. In general, there will be a set of $\beta$ 's representing the contribution of different groups of coupled symbols (as in the following example).

\subsubsection{A QOSTBC with $n_{T}=8$}

Consider the rate-one full-diversity $n_{T}=L=8$ QOSTBC proposed in [11]:

$$
\begin{aligned}
\mathbf{C} & =\left[c_{i j}\right]_{8 \times 8} \\
& =\left[\begin{array}{cccccccccc}
x_{1} & -x_{2} & -x_{3}^{*} & -x_{4}^{*} & -x_{5}^{*} & -x_{6}^{*} & -x_{7} & -x_{8} \\
x_{2} & x_{1} & -x_{4}^{*} & x_{3}^{*} & -x_{6}^{*} & x_{5}^{*} & x_{8} & -x_{7} \\
x_{3} & x_{4} & x_{1}^{*} & -x_{2}^{*} & -x_{7}^{*} & -x_{8}^{*} & x_{5} & x_{6} \\
x_{4} & -x_{3} & x_{2}^{*} & x_{1}^{*} & -x_{8}^{*} & x_{7}^{*} & -x_{6} & x_{5} \\
x_{5} & x_{6} & x_{7}^{*} & x_{8}^{*} & x_{1}^{*} & -x_{2}^{*} & -x_{3} & -x_{4} \\
x_{6} & -x_{5} & x_{8}^{*} & -x_{7}^{*} & x_{2}^{*} & x_{1}^{*} & x_{4} & -x_{3} \\
x_{7} & -x_{8} & -x_{5}^{*} & x_{6}^{*} & x_{3}^{*} & -x_{4}^{*} & x_{1} & x_{2} \\
x_{8} & x_{7} & -x_{6}^{*} & -x_{5}^{*} & x_{4}^{*} & x_{3}^{*} & -x_{2} & x_{1}
\end{array}\right] .
\end{aligned}
$$

By generating the coupled symbols in the ML metric expression, the decoding complexity can be analyzed. Using straightforward simplification:

$$
\begin{aligned}
& 2 \sum_{j=1}^{n_{T}} \sum_{m=j+1}^{n_{T}}\left(\Re\left\{\left\langle\mathbf{h}_{j}, \mathbf{h}_{m}\right\rangle\right\} \Re\left\{\left\langle\mathbf{c}^{(m)}, \mathbf{c}^{(j)}\right\rangle\right\}+\Im\left\{\left\langle\mathbf{h}_{j}, \mathbf{h}_{m}\right\rangle\right\} \Im\left\{\left\langle\mathbf{c}^{(m)}, \mathbf{c}^{(j)}\right\rangle\right\}\right) \\
& =\beta_{12} g\left(x_{1}, x_{2}\right)+\beta_{78} g\left(x_{7}, x_{8}\right)+\beta_{17} g\left(x_{1}, x_{7}\right)+\beta_{28} g\left(x_{2}, x_{8}\right)+\beta_{18} g\left(x_{1}, x_{8}\right)+\beta_{27} g\left(x_{2}, x_{7}\right) \\
& +\beta_{34} g\left(x_{3}, x_{4}\right)+\beta_{56} g\left(x_{5}, x_{6}\right)+\beta_{35} g\left(x_{3}, x_{5}\right)+\beta_{46} g\left(x_{4}, x_{6}\right)+\beta_{36} g\left(x_{3}, x_{6}\right)+\beta_{45} g\left(x_{4}, x_{5}\right)
\end{aligned}
$$


where $g\left(x_{a}, x_{b}\right)=\Re\left\{x_{a}\right\} \Im\left\{x_{b}\right\}-\Im\left\{x_{a}\right\} \Re\left\{x_{b}\right\}$. The $\beta$ values are as follows:

$$
\begin{aligned}
& \beta_{12}=4\left(\Im\left\{\left\langle\mathbf{h}_{1}, \mathbf{h}_{2}\right\rangle\right\}-\Im\left\{\left\langle\mathbf{h}_{3}, \mathbf{h}_{4}\right\rangle\right\}-\Im\left\{\left\langle\mathbf{h}_{5}, \mathbf{h}_{6}\right\rangle\right\}-\Im\left\{\left\langle\mathbf{h}_{7}, \mathbf{h}_{8}\right\rangle\right\}\right), \\
& \beta_{78}=4\left(-\Im\left\{\left\langle\mathbf{h}_{1}, \mathbf{h}_{2}\right\rangle\right\}-\Im\left\{\left\langle\mathbf{h}_{3}, \mathbf{h}_{4}\right\rangle\right\}-\Im\left\{\left\langle\mathbf{h}_{5}, \mathbf{h}_{6}\right\rangle\right\}+\Im\left\{\left\langle\mathbf{h}_{7}, \mathbf{h}_{8}\right\rangle\right\}\right), \\
& \beta_{17}=4\left(\Im\left\{\left\langle\mathbf{h}_{1}, \mathbf{h}_{7}\right\rangle\right\}+\Im\left\{\left\langle\mathbf{h}_{2}, \mathbf{h}_{8}\right\rangle\right\}-\Im\left\{\left\langle\mathbf{h}_{3}, \mathbf{h}_{5}\right\rangle\right\}+\Im\left\{\left\langle\mathbf{h}_{4}, \mathbf{h}_{6}\right\rangle\right\}\right), \\
& \beta_{28}=4\left(\Im\left\{\left\langle\mathbf{h}_{1}, \mathbf{h}_{7}\right\rangle\right\}+\Im\left\{\left\langle\mathbf{h}_{2}, \mathbf{h}_{8}\right\rangle\right\}+\Im\left\{\left\langle\mathbf{h}_{3}, \mathbf{h}_{5}\right\rangle\right\}-\Im\left\{\left\langle\mathbf{h}_{4}, \mathbf{h}_{6}\right\rangle\right\}\right), \\
& \beta_{18}=4\left(\Im\left\{\left\langle\mathbf{h}_{1}, \mathbf{h}_{8}\right\rangle\right\}-\Im\left\{\left\langle\mathbf{h}_{2}, \mathbf{h}_{7}\right\rangle\right\}-\Im\left\{\left\langle\mathbf{h}_{3}, \mathbf{h}_{6}\right\rangle\right\}-\Im\left\{\left\langle\mathbf{h}_{4}, \mathbf{h}_{5}\right\rangle\right\}\right), \\
& \beta_{27}=4\left(-\Im\left\{\left\langle\mathbf{h}_{1}, \mathbf{h}_{8}\right\rangle\right\}+\Im\left\{\left\langle\mathbf{h}_{2}, \mathbf{h}_{7}\right\rangle\right\}-\Im\left\{\left\langle\mathbf{h}_{3}, \mathbf{h}_{6}\right\rangle\right\}-\Im\left\{\left\langle\mathbf{h}_{4}, \mathbf{h}_{5}\right\rangle\right\}\right), \\
& \beta_{34}=4\left(-\Im\left\{\left\langle\mathbf{h}_{1}, \mathbf{h}_{2}\right\rangle\right\}+\Im\left\{\left\langle\mathbf{h}_{3}, \mathbf{h}_{4}\right\rangle\right\}-\Im\left\{\left\langle\mathbf{h}_{5}, \mathbf{h}_{6}\right\rangle\right\}-\Im\left\{\left\langle\mathbf{h}_{7}, \mathbf{h}_{8}\right\rangle\right\}\right), \\
& \beta_{56}=4\left(-\Im\left\{\left\langle\mathbf{h}_{1}, \mathbf{h}_{2}\right\rangle\right\}-\Im\left\{\left\langle\mathbf{h}_{3}, \mathbf{h}_{4}\right\rangle\right\}+\Im\left\{\left\langle\mathbf{h}_{5}, \mathbf{h}_{6}\right\rangle\right\}-\Im\left\{\left\langle\mathbf{h}_{7}, \mathbf{h}_{8}\right\rangle\right\}\right), \\
& \beta_{35}=4\left(-\Im\left\{\left\langle\mathbf{h}_{1}, \mathbf{h}_{7}\right\rangle\right\}+\Im\left\{\left\langle\mathbf{h}_{2}, \mathbf{h}_{8}\right\rangle\right\}+\Im\left\{\left\langle\mathbf{h}_{3}, \mathbf{h}_{5}\right\rangle\right\}+\Im\left\{\left\langle\mathbf{h}_{4}, \mathbf{h}_{6}\right\rangle\right\}\right), \\
& \beta_{46}=4\left(\Im\left\{\left\langle\mathbf{h}_{1}, \mathbf{h}_{7}\right\rangle\right\}-\Im\left\{\left\langle\mathbf{h}_{2}, \mathbf{h}_{8}\right\rangle\right\}+\Im\left\{\left\langle\mathbf{h}_{3}, \mathbf{h}_{5}\right\rangle\right\}+\Im\left\{\left\langle\mathbf{h}_{4}, \mathbf{h}_{6}\right\rangle\right\}\right), \\
& \beta_{36}=4\left(-\Im\left\{\left\langle\mathbf{h}_{1}, \mathbf{h}_{8}\right\rangle\right\}-\Im\left\{\left\langle\mathbf{h}_{2}, \mathbf{h}_{7}\right\rangle\right\}+\Im\left\{\left\langle\mathbf{h}_{3}, \mathbf{h}_{6}\right\rangle\right\}-\Im\left\{\left\langle\mathbf{h}_{4}, \mathbf{h}_{5}\right\rangle\right\}\right), \\
& \beta_{45}=4\left(-\Im\left\{\left\langle\mathbf{h}_{1}, \mathbf{h}_{8}\right\rangle\right\}-\Im\left\{\left\langle\mathbf{h}_{2}, \mathbf{h}_{7}\right\rangle\right\}-\Im\left\{\left\langle\mathbf{h}_{3}, \mathbf{h}_{6}\right\rangle\right\}+\Im\left\{\left\langle\mathbf{h}_{4}, \mathbf{h}_{5}\right\rangle\right\}\right) .
\end{aligned}
$$

Clearly, the coupling in the ML metric expression is more complex than in the previous example. Note that the metric can only be separated into two non-interacting terms: $f_{1278}\left(x_{1}, x_{2}, x_{7}, x_{8}\right)+f_{3456}\left(x_{3}, x_{4}, x_{5}, x_{6}\right)$, each requiring the simultaneous detection of 8 real entities. Hence, the ML decoding complexity is of order 8 .

\subsubsection{A New QOSTBC with Order 4 ML Decoding Complexity}

In [11] a rate-one full-diversity QOSTBC for 8 antennas is proposed with a metric expression that can only be decoupled into two terms: $f_{1278}\left(x_{1}, x_{2}, x_{7}, x_{8}\right)+$ 
$f_{3456}\left(x_{3}, x_{4}, x_{5}, x_{6}\right)$. Yet rate-one full-diversity single-symbol decodable QOSTBCs exist for 4 transmit antennas (e.g. [12]). This suggests that there exists a rate-one full-diversity QOSTBC for 8 antennas with a metric that can be decoupled into more than two independent terms. How can this be constructed, and how can (2.4) be used as a design tool to assist in the construction? If one wanted to design STBCs guaranteed to have low decoding complexity, one could use group-decodable theory, such as that in [29]. However, if one constructs a new STBC based on any other criterion or technique, the "coupled symbols" term in (2.4) provides a quick way to judge the decoding complexity of the new STBC.

Consider taking the MDC-QOSTBC in [12, eqn. (7)] and constructing a rate-one 8 antenna QOSTBC using the modular/recursive technique outlined in [10]:

$$
\mathbf{C}=\left[\begin{array}{cc}
\mathbf{C}_{1234} & -\mathbf{C}_{5678}^{*} \\
\mathbf{C}_{5678} & \mathbf{C}_{1234}^{*}
\end{array}\right],
$$

where

$$
\begin{aligned}
& \mathbf{C}_{1234}= {\left[\begin{array}{cccc}
\Re\left\{x_{1}\right\}+j \Re\left\{x_{3}\right\} & -\Re\left\{x_{2}\right\}+j \Re\left\{x_{4}\right\} & -\Im\left\{x_{1}\right\}+j \Im\left\{x_{3}\right\} & \Im\left\{x_{2}\right\}+j \Im\left\{x_{4}\right\} \\
\Re\left\{x_{2}\right\}+j \Re\left\{x_{4}\right\} & \Re\left\{x_{1}\right\}-j \Re\left\{x_{3}\right\} & -\Im\left\{x_{2}\right\}+j \Im\left\{x_{4}\right\} & -\Im\left\{x_{1}\right\}-j \Im\left\{x_{3}\right\} \\
-\Im\left\{x_{1}\right\}+j \Im\left\{x_{3}\right\} & \Im\left\{x_{2}\right\}+j \Im\left\{x_{4}\right\} & \Re\left\{x_{1}\right\}+j \Re\left\{x_{3}\right\} & -\Re\left\{x_{2}\right\}+j \Re\left\{x_{4}\right\} \\
-\Im\left\{x_{2}\right\}+j \Im\left\{x_{4}\right\} & -\Im\left\{x_{1}\right\}-j \Im\left\{x_{3}\right\} & \Re\left\{x_{2}\right\}+j \Re\left\{x_{4}\right\} & \Re\left\{x_{1}\right\}-j \Re\left\{x_{3}\right\}
\end{array}\right] } \\
& \mathbf{C}_{5678}=\left[\begin{array}{cccc}
\Re\left\{x_{5}\right\}+j \Re\left\{x_{7}\right\} & -\Re\left\{x_{6}\right\}+j \Re\left\{x_{8}\right\} & -\Im\left\{x_{5}\right\}+j \Im\left\{x_{7}\right\} & \Im\left\{x_{6}\right\}+j \Im\left\{x_{8}\right\} \\
\Re\left\{x_{6}\right\}+j \Re\left\{x_{8}\right\} & \Re\left\{x_{5}\right\}-j \Re\left\{x_{7}\right\} & -\Im\left\{x_{6}\right\}+j \Im\left\{x_{8}\right\} & -\Im\left\{x_{5}\right\}-j \Im\left\{x_{7}\right\} \\
-\Im\left\{x_{5}\right\}+j \Im\left\{x_{7}\right\} & \Im\left\{x_{6}\right\}+j \Im\left\{x_{8}\right\} & \Re\left\{x_{5}\right\}+j \Re\left\{x_{7}\right\} & -\Re\left\{x_{6}\right\}+j \Re\left\{x_{8}\right\} \\
-\Im\left\{x_{6}\right\}+j \Im\left\{x_{8}\right\} & -\Im\left\{x_{5}\right\}-j \Im\left\{x_{7}\right\} & \Re\left\{x_{6}\right\}+j \Re\left\{x_{8}\right\} & \Re\left\{x_{5}\right\}-j \Re\left\{x_{7}\right\}
\end{array}\right] .
\end{aligned}
$$


Using (2.4) as a design tool to quickly check the degree of coupling:

$$
\begin{aligned}
& 2 \sum_{j=1}^{n_{T}} \sum_{m=j+1}^{n_{T}}\left(\Re\left\{\left\langle\mathbf{h}_{j}, \mathbf{h}_{m}\right\rangle\right\} \Re\left\{\left\langle\mathbf{c}^{(m)}, \mathbf{c}^{(j)}\right\rangle\right\}+\Im\left\{\left\langle\mathbf{h}_{j}, \mathbf{h}_{m}\right\rangle\right\} \Im\left\{\left\langle\mathbf{c}^{(m)}, \mathbf{c}^{(j)}\right\rangle\right\}\right) \\
& =\beta_{1}\left(-\gamma_{1}\left(x_{1}, x_{6}\right)-\gamma_{1}\left(x_{2}, x_{5}\right)+\gamma_{1}\left(x_{3}, x_{8}\right)+\gamma_{1}\left(x_{4}, x_{7}\right)\right) \\
& +\beta_{2}\left(\gamma_{2}\left(x_{1}, x_{6}\right)-\gamma_{2}\left(x_{2}, x_{5}\right)+\gamma_{2}\left(x_{3}, x_{8}\right)-\gamma_{2}\left(x_{4}, x_{7}\right)\right) \\
& +\beta_{3}\left(\gamma_{3}\left(x_{1}, x_{6}\right)-\gamma_{3}\left(x_{2}, x_{5}\right)-\gamma_{3}\left(x_{3}, x_{8}\right)+\gamma_{3}\left(x_{4}, x_{7}\right)\right)
\end{aligned}
$$

where $\gamma_{1}\left(x_{a}, x_{b}\right)=\Re\left\{x_{a}\right\} \Im\left\{x_{a}\right\}+\Re\left\{x_{b}\right\} \Im\left\{x_{b}\right\}, \gamma_{2}\left(x_{a}, x_{b}\right)=\Re\left\{x_{a}\right\} \Re\left\{x_{b}\right\}+\Im\left\{x_{a}\right\} \Im\left\{x_{b}\right\}$, and $\gamma_{3}\left(x_{a}, x_{b}\right)=\Re\left\{x_{a}\right\} \Im\left\{x_{b}\right\}+\Im\left\{x_{a}\right\} \Re\left\{x_{b}\right\}$. The $\beta$ values are as follows:

$$
\begin{aligned}
& \beta_{1}=4\left(\Re\left\{\left\langle\mathbf{h}_{1}, \mathbf{h}_{3}\right\rangle\right\}+\Re\left\{\left\langle\mathbf{h}_{2}, \mathbf{h}_{4}\right\rangle\right\}+\Re\left\{\left\langle\mathbf{h}_{5}, \mathbf{h}_{7}\right\rangle\right\}+\Re\left\{\left\langle\mathbf{h}_{6}, \mathbf{h}_{8}\right\rangle\right\}\right) \\
& \beta_{2}=4\left(\Re\left\{\left\langle\mathbf{h}_{1}, \mathbf{h}_{6}\right\rangle\right\}-\Re\left\{\left\langle\mathbf{h}_{2}, \mathbf{h}_{5}\right\rangle\right\}+\Re\left\{\left\langle\mathbf{h}_{3}, \mathbf{h}_{8}\right\rangle\right\}-\Re\left\{\left\langle\mathbf{h}_{4}, \mathbf{h}_{7}\right\rangle\right\}\right) \\
& \beta_{3}=4\left(-\Re\left\{\left\langle\mathbf{h}_{1}, \mathbf{h}_{8}\right\rangle\right\}+\Re\left\{\left\langle\mathbf{h}_{2}, \mathbf{h}_{7}\right\rangle\right\}-\Re\left\{\left\langle\mathbf{h}_{3}, \mathbf{h}_{6}\right\rangle\right\}+\Re\left\{\left\langle\mathbf{h}_{4}, \mathbf{h}_{5}\right\rangle\right\}\right)
\end{aligned}
$$

Note that this metric expression can be split into 4 independent terms: $f_{16}\left(x_{1}, x_{6}\right)+$ $f_{25}\left(x_{2}, x_{5}\right)+f_{38}\left(x_{3}, x_{8}\right)+f_{47}\left(x_{4}, x_{7}\right)$. Also, the set of $\beta$ values associated with the coupled terms is smaller. Hence, this new $n_{T}=L=8$ QOSTBC has a lower decoding complexity than that proposed in [11].

As shown in Figure 2.1, with no constellation rotation the performance of (2.5) is worse than that proposed in [11]. However, by drawing $\left(x_{5}, x_{6}, x_{7}, x_{8}\right)$ from a constellation rotated by $\pi / 8$ (found through simulations), 2.5 can achieve full-diversity. In Chapter 4, constellation rotation is discussed in detail, and the challenge of analytically justifying the $\pi / 8$ rotation angle is examined. 


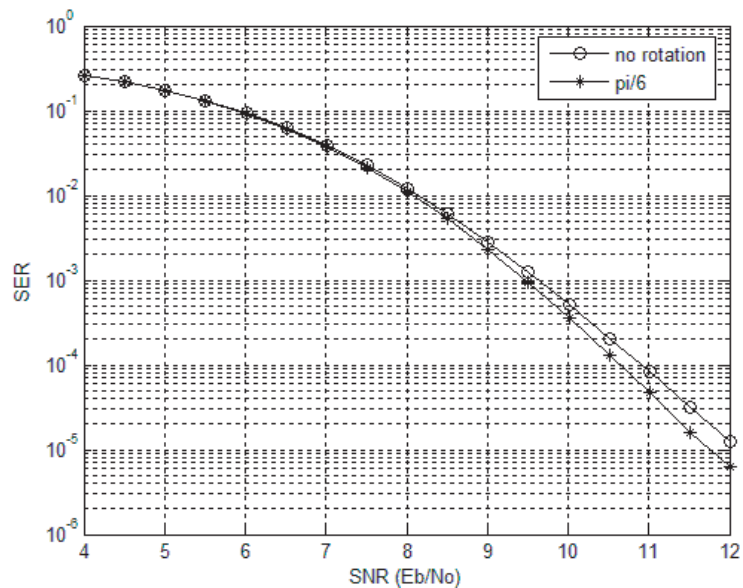

(a)

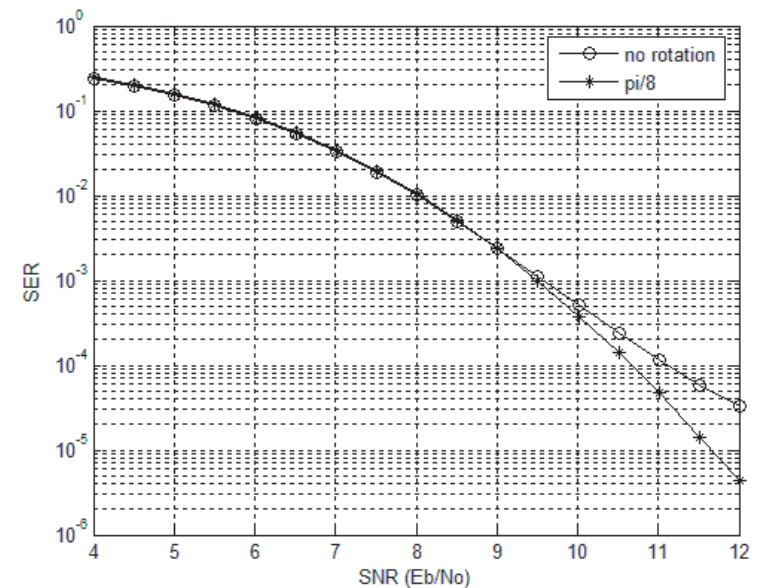

(b)

Figure 2.1: SER for $n_{T}=8$, rate-one QOSTBCs with 4-QAM, $n_{R}=1$, and Rayleigh fading. (a) QOSTBC proposed in [11] with its optimum constellation rotation angles $\theta=\pi / 6$ and $\theta^{\prime}=\pi / 4$ (see [11, eqn. (7)]). (b) New QOSTBC constructed (2.5) where $x_{5}, x_{6}, x_{7}$, and $x_{8}$ are drawn from a constellation rotated by $\pi / 8$.

\subsection{Conclusion}

In this chapter a new vectorization was proposed for a linear STBC that maintains the redundancy in the STBC matrix $\mathbf{C}$. This new vectorization was used to express the ML metric expression in a generic form that separates the coupled and decoupled portions of the metric and makes simplification substantially easier. Examples of metric simplification were then considered, and a new 8 antenna rate-one full-diversity QOSTBC with lower decoding complexity was constructed. The "coupled symbols" term in (2.4) was used as a design tool to quickly check the degree of decoupling that resulted when using the construction technique. 


\section{Chapter 3}

\section{ML and sub-ML Decoding of QOSTBCs}

\subsection{Introduction}

As discussed in Section 1.4.3, it is desirable to employ OSTBCs since the ML metric expression can be completely decoupled, thereby reducing the decoding complexity to a linear function of the number of transmitted symbols, $n_{s}$. However, if the underlying constellation is complex, rate-one full-diversity OSTBCs do not exist for more than 2 transmit antennas. For this reason, QOSTBCs have been developed by relaxing the constraint of linear decoding. These STBCs have better error performance, but their metric can only be partially decoupled into groups of symbols. Therefore, decoding QOSTBCs is more complex. The received symbols can only be partially decoupled, and the complexity scales exponentially with $n_{s}$. The ML decoding complexity now becomes a concern; depending on the size of $n_{s}$, the underlying constellation, and the degree of coupling in the metric expression, performing a brute-force exhaustive 
search may become prohibitively complex. This raises the following questions:

1. Assuming we are interested in ML decoding, for the collection of QOSTBCs proposed in the literature (e.g $[5,10-12])$, when is it best to perform an exhaustive search using a simplified and partially decoupled metric expression, and when should an efficient implementation of the sphere decoding algorithm (SDA) be applied? The answer should depend on a number of different factors, including the number of transmitted symbols, $n_{s}$, the number of receive antennas, $n_{R}$, the rate and diversity of the STBC, and the size of the underlying constellation. How do these parameters affect the boundary?

2. When is it a good choice to decode QOSTBCs using sub-optimal decoding algorithms? How do the sub-optimal algorithms primarily used in layered MIMO systems (Section 1.5.1) translate to QOSTBCs in terms of the complexity versus error performance tradeoff? Can we develop a new family of sub-optimal decoding algorithms for QOSTBCs using the metric expression in 2.4 ?

The above questions are examined and answered in this chapter. In Section 3.2 , the question of when to use the SDA is considered. Then in Section 3.3, the suboptimal decoding of QOSTBCs is considered.

\subsection{Decoding Algorithms for STBCs: SDA versus an Exhaustive Search}

The advantage of QOSTBCs is that they offer better error performance than OSTBCs, while giving up only some orthogonality. Therefore, for QOSTBCs with a small number of transmit antennas and a small underlying constellation, an exhaustive 
search will be low-complexity. On the other hand, the non-orthogonality of QOSTBCs means that the SDA will eventually be the better choice when the number of transmit antennas and/or underlying constellation becomes large. In what follows, the ML decoding complexity of a collection of QOSTBCs are analyzed to provide direction as to when to switch from an exhaustive search to the SDA. Conclusions will then be drawn from the simulation results.

Before discussing the results, a few specifics about the SDA used in the simulations need to be mentioned. The SDA implementation was based on the SchnorrEuchner enumeration. Specifically, [17, Algorithm II, Smart Implementation] was implemented. The metric was separated into as many independent Euclidean norms as possible as in [25], and smaller-dimensional SDAs were applied to each norm separately.

\subsubsection{MDC-QOSTBCs}

Let us first consider the 4 and 8 transmit antenna minimum-decoding-complexity QOSTBCs (MDC-QOSTBCs) proposed in [12]. Both of these QOSTBCs can be made full-diversity using an optimal constellation rotation. The 4 transmit antenna MDC-QOSTBC is rate-one, whereas the 8 transmit antenna MDC-QOSTBC can only have a maximum rate of $3 / 4$. Since these QOSTBCs are minimum-decodingcomplexity, an exhaustive search should likely outperform the SDA. As an example, consider the $n_{T}=8 \mathrm{MDC}-\mathrm{QOSTBC}$ with 16 -QAM, $n_{R}=4$, and rate $3 / 4$. The metric can be separated into 6 independent terms: $f_{1}\left(x_{1}\right)+f_{2}\left(x_{2}\right)+\ldots+f_{6}\left(x_{6}\right)$. Performing an exhaustive search on this metric expression requires less than 1300 flops. This is less than the number of flops required to perform the QR factorization in the SDA 
(using [30, Algorithm 6.2-1]), even if the metric is written as 6 independent Euclidean norms and 6 separate SDAs are applied. As expected, an exhaustive search is the better choice. However, it should be noted that the complexity of the SDA is a lot less sensitive to the underlying constellation than an exhaustive search. Therefore, when implementing MDC-QOSTBCs with 64-QAM and higher, one must be careful in determining the decoding scheme; depending on $n_{s}$ and $n_{R}$, the SDA will start to become a desirable alternative.

\subsubsection{Rate-One Full-Diversity QOSTBCs in [11]}

Let us next consider the 4 and 8 transmit antenna QOSTBCs proposed in [11]. Both of these QOSTBCs are rate-one, and an optimal constellation rotation can be applied to both to achieve full-diversity. The full-diversity 4 transmit antenna QOSTBC in [11] is equivalent to the full-diversity 4 transmit antenna MDC-QOSTBC in [12], and so for this QOSTBC an exhaustive search is the better choice. The ML metric for the rateone full-diversity 8 antenna QOSTBC in [11] can be decoupled into two independent terms: $f_{1278}\left(x_{1}, x_{2}, x_{7}, x_{8}\right)+f_{3456}\left(x_{3}, x_{4}, x_{5}, x_{6}\right)$. Therefore, two independent SDAs may be applied. In Figure 3.1, the decoding complexity of an exhaustive search versus the average decoding complexity using two independent SDAs is compared over the SNRs of interest. When 4-QAM is the underlying constellation, an exhaustive search is likely the better choice; however, this depends on the value of $n_{R}$. If 16-QAM is used, the SDA performance is substantially better. One interesting observation is that the complexity of the exhaustive search is much less sensitive to $n_{R}$ than the SDA. Therefore, in scenarios when the complexities of both algorithms are on the same order of magnitude, an exhaustive search should be favoured for large $n_{R}$. 


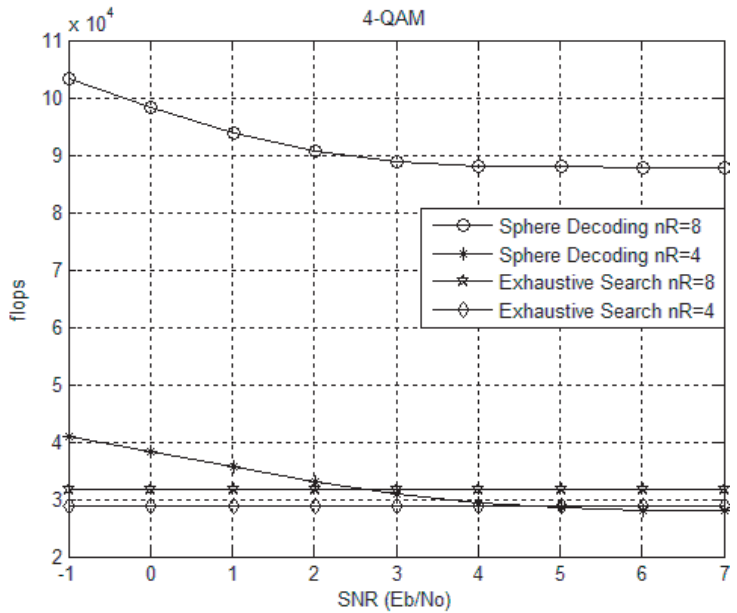

(a)

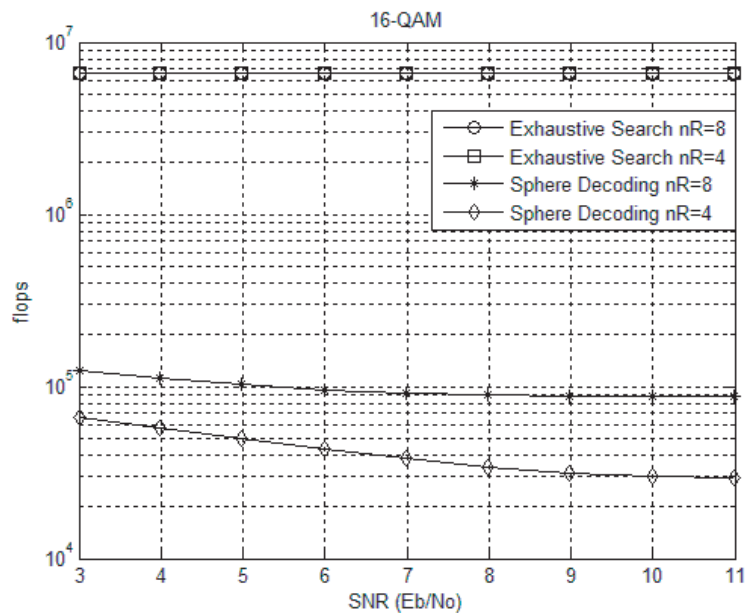

(b)

Figure 3.1: Complexity comparison for $n_{T}=8$, rate-one QOSTBC in [11] for a Rayleigh fading channel. (a) 4-QAM. (b) 16-QAM.

\subsubsection{QOSTBC Constructed in (2.5)}

Let us now consider the rate-one 8 transmit antenna QOSTBC constructed in (2.5). It can also be rotated to achieve full-diversity. Additionally, its metric can be decoupled into four independent terms: $f_{16}\left(x_{1}, x_{6}\right)+f_{25}\left(x_{2}, x_{5}\right)+f_{38}\left(x_{3}, x_{8}\right)+f_{47}\left(x_{4}, x_{7}\right)$. This means that the metric can be written as 4 independent Euclidean norms and 4 separate SDAs can be applied. Of course, it also means performing an exhaustive search will be feasible in more cases. In Figure 3.2 , the decoding complexity of an exhaustive search versus the average decoding complexity using four independent SDAs is compared over the SNR of interest. It is more efficient to perform an exhaustive search for both 4-QAM and 16-QAM. However, for underlying constellations with more than 16 points, the SDA becomes the better choice. 


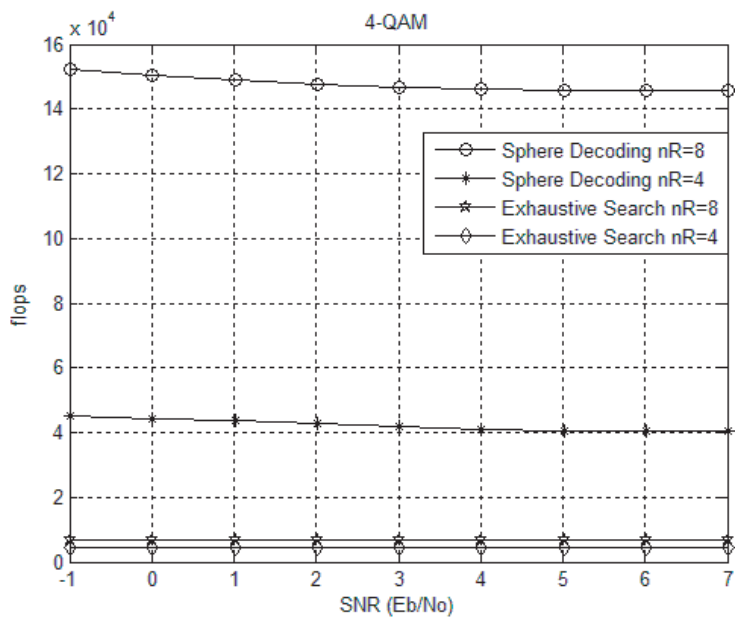

(a)

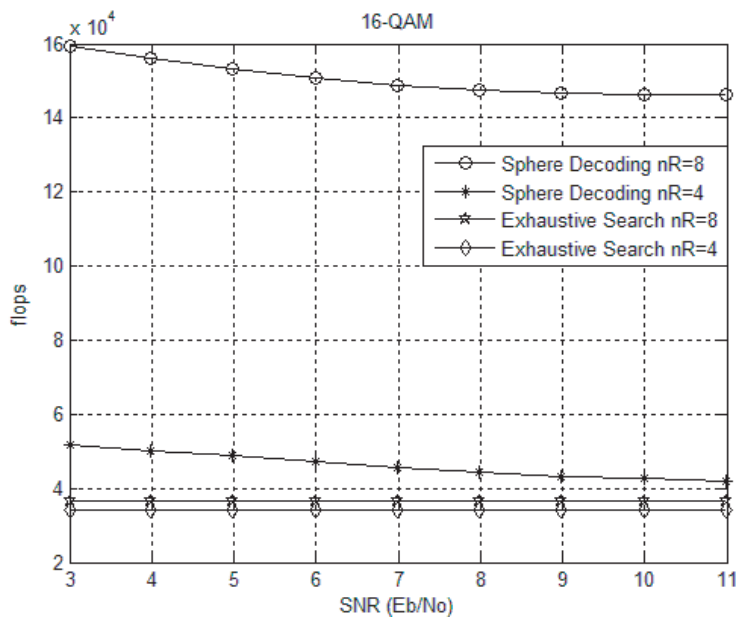

(b)

Figure 3.2: Complexity comparison for $n_{T}=8$, rate-one QOSTBC constructed in (2.5) for a Rayleigh fading channel. (a) 4-QAM. (b) 16-QAM.

\subsubsection{Conclusion}

In conclusion, the following observations can be made: 1) By choosing an appropriate QOSTBC and appropriate constellation rotation, rate-one full-diversity QOSTBCs with up to 8 transmit antennas and 16-QAM can be decoded efficiently by performing an exhaustive search on the simplified metric expression in (2.4). 2) The complexity of an exhaustive search is not very sensitive to $n_{R}$. 3) The complexity of an exhaustive search is independent of the SNR; therefore, for break-even scenarios an exhaustive search is favoured. 4) The SDA is a better choice for decoding QOSTBCs when both $n_{T}$ and the underlying constellation are large; while in other cases, an exhaustive search may be considerably faster. 5) For cases in between (e.g. a rate-one fulldiversity QOSTBC with $n_{T}=4$ and 64-QAM), one must study the complexity of each decoder in light of the metric expression in (2.4). 


\subsection{Sub-optimal Decoding Algorithms}

Let us now consider decoding QOSTBCs using sub-optimal decoding algorithms, i.e., algorithms that sacrifice error performance for a reduction in decoding complexity. Obviously, if the STBC is orthogonal, there is no need for a sub-optimal decoding algorithm; the complete decoupling of the metric allows for a low-complexity exhaustive search. But for some QOSTBCs, an optimal decoding algorithm may be too demanding for the application. How does one approach the sub-optimal decoding of non-orthogonal STBCs? This section will focus on this question in the context of QOSTBCs.

\subsubsection{Previous Works}

There are relatively few papers that address the sub-optimal decoding of QOSTBCs. This is because QOSTBCs that decouple into pairs of symbols (such as that in [10]) are still conducive to decoding via an exhaustive search. It is only when both $n_{T}$ and the underlying constellation become large that sub-optimal schemes become attractive.

Before studying sub-optimal algorithms, it is worth highlighting the fact that there are other techniques that offer ML performance while reducing the overall decoding complexity. For example, in $[31,32] \mathrm{ML}$ decoders for the $n_{T}=L=4$ QOSTBC in [10] are proposed. These decoders perform a QR decomposition on the channel matrix to save computations in the exhaustive search portion of the algorithm. The complexity analysis given in $[31,32]$ assumes slow fading, and therefore the complexity of the QR decomposition is ignored. If slow fading is assumed, the decoders offer lower complexity ML performance than an exhaustive search using a simplified, partially decoupled metric. However, for quasi-static fading the decoders in [31,32] are 
higher-complexity. Alternatively, if ML decoding is being performed via the SDA, as mentioned in Section 1.5.2, [25] offers a technique to reduce the complexity of the SDA by taking advantage of the partial decoupling in the metric expression.

However, if one wanted to apply sub-optimal algorithms to trade-off the error performance of QOSTBCs for a reduction in complexity, there are few previous studies to provide direction. A natural approach is to apply the sub-optimal decoding techniques used in layered MIMO systems. These algorithms are built around solving the unconstrained least-squares problem and slicing to the nearest constellation point (see Section 1.5.1]. For example, in [33] the error performance of the $n_{T}=L=4$ QOSTBC is analyzed for a receiver that performs lattice-reduction-aided zero-forcing; however, no complexity analysis is provided. In [34] the decoding of QOSTBCs using simple sub-optimal linear receivers (e.g. zero-forcing) is studied, but only cyclic STBCs and the extended Alamouti code are considered; there is no complexity analysis and no extension to more general families of QOSTBCs. In [32] a zero-forcing QR-decomposition-based decoder is implemented in simulations, but the complexity analysis assumes slow fading and therefore ignores preprocessing.

\subsubsection{Decoding QOSTBCs using a simple linear receiver}

Let us consider the decoding of QOSTBCs using simple zero-forcing. As with the rest of the thesis, we will assume quasi-static fading. For a QOSTBC with (vectorized) system model $\mathbf{r}^{\prime}=\mathbf{F}^{\prime} \mathbf{x}^{\prime}+\mathbf{n}^{\prime}$, the zero-forcing solution is found by slicing the real $2 n_{s} \times 1$ vector, $\hat{\mathbf{x}}$, to the nearest constellation point, where $\hat{\mathbf{x}}$ is solved as follows:

$$
\hat{\mathbf{x}}=\mathbf{F}^{\dagger} \mathbf{r}^{\prime}
$$




\begin{tabular}{|c|c|c|c|}
\hline $\begin{array}{c}\text { QOSTBCs in [11] } \\
\text { with 16-QAM }\end{array}$ & Zero-Forcing & Exhaustive Search & Sphere Decoding \\
\hline$n_{T}=L=n_{R}=4$ & 2304 & 360 & N/A \\
\hline$n_{T}=L=n_{R}=8$ & 17408 & $\approx 7.5 \times 10^{5}$ & $\begin{array}{c}15500 \rightarrow 11000 \\
\text { from Figure 3.1) }\end{array}$ \\
\hline
\end{tabular}

Table 3.1: A complexity comparison (in terms of number of flops per information symbol) among zero-forcing, an exhaustive search, and the sphere decoding algorithm for the QOSTBCs in [11].

where $\mathbf{F}^{\dagger}$ is the Penrose-Moore Pseudo-Inverse:

$$
\mathbf{F}^{\dagger}=\left(\mathbf{F}^{\prime T} \mathbf{F}^{\prime}\right)^{-1} \mathbf{F}^{\prime T} .
$$

Note that for QOSTBCs, $\mathbf{F}^{\prime}$ will not be ill-conditioned. If $\mathbf{F}^{\prime}$ is an $n \times m$ matrix, the number of flops to perform zero-forcing is

$$
4 m^{2} n+2 m n+m^{3}
$$

where the inverse operation $(\cdot)^{-1}$ has been estimated to take $m^{3}$ flops. For example, the $n_{T}=L=n_{R}=4$ QOSTBC in [11] takes 9216 flops to be detected using zeroforcing.

Figure 3.3 shows the zero-forcing BER curves for the 4 and 8 transmit antenna QOSTBCs proposed in [11]. Rayleigh fading is assumed and the underlying constellation is 16-QAM. It is clear from Figure 3.3 that simple zero-forcing performs remarkably well for QOSTBCs. Unlike in the spatial multiplexing case, there is no loss in diversity gain. These curves are consistent with similar studies, such as that in [33] 1]. The fact that the diversity gain is preserved by the zero-forcing algorithm

\footnotetext{
${ }^{1}$ Although the receiver in [33] performs lattice-reduction-aided zero-forcing, the study goes on to argue that performing lattice reduction has little effect when decoding QOSTBCs.
} 


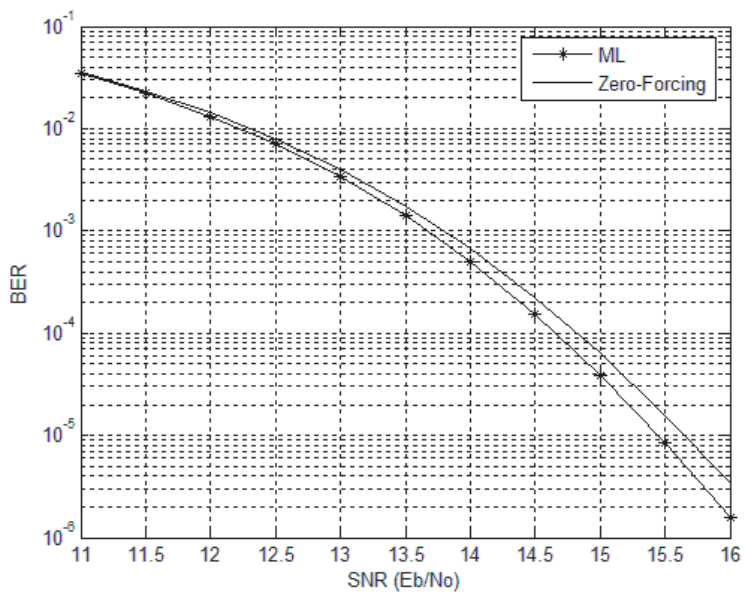

(a) $\mathrm{nT}=\mathrm{nR}=\mathrm{L}=4$

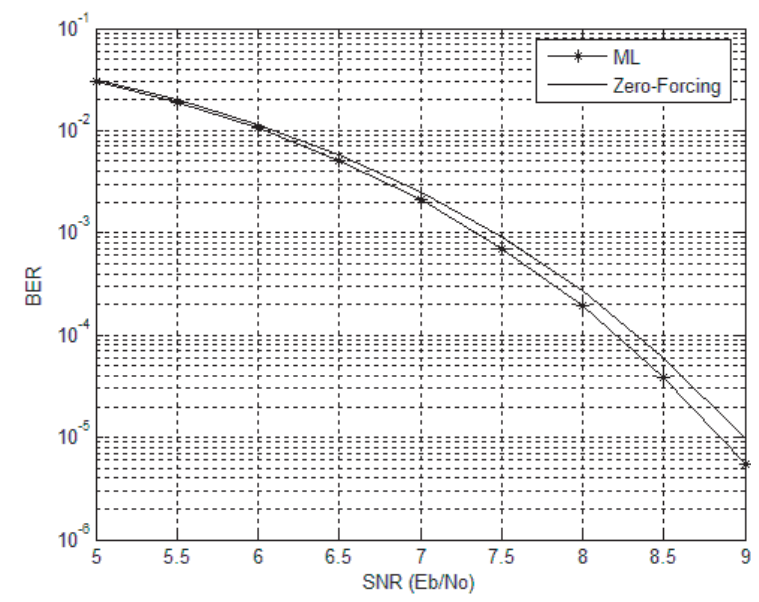

(b) $\mathrm{nT}=\mathrm{nR}=\mathrm{L}=8$

Figure 3.3: A BER comparison of ML decoding versus zero-forcing for the 4 and 8 antenna QOSTBCs proposed in [11]. Rayleigh fading is assumed and the underlying constellation is 16-QAM.

can be justified by the structure of $\mathbf{F}^{\prime}$ : for QOSTBCs, the columns of $\mathbf{F}^{\prime}$ are still highly orthogonal. The situation is similar to having a matrix with non-orthogonal columns and then performing lattice reduction to increase orthogonality. In this case, it is shown in [35] that lattice reduction with a simple sub-optimal decoding algorithm achieves the same diversity as ML decoding.

Even though zero-forcing performs well, its complexity is not negligible. The complexity of performing the zero-forcing algorithm (in terms of actual number of flops per information symbol) versus an exhaustive search and sphere decoding is shown in Table 3.1. For the 4 transmit antenna QOSTBC, it is less complex to perform an exhaustive search on a simplified and partially decoupled metric. For the 8 transmit antenna QOSTBC, zero-forcing is on the same order of magnitude as the SDA. One advantage of zero-forcing, though, is that its complexity is independent of both 


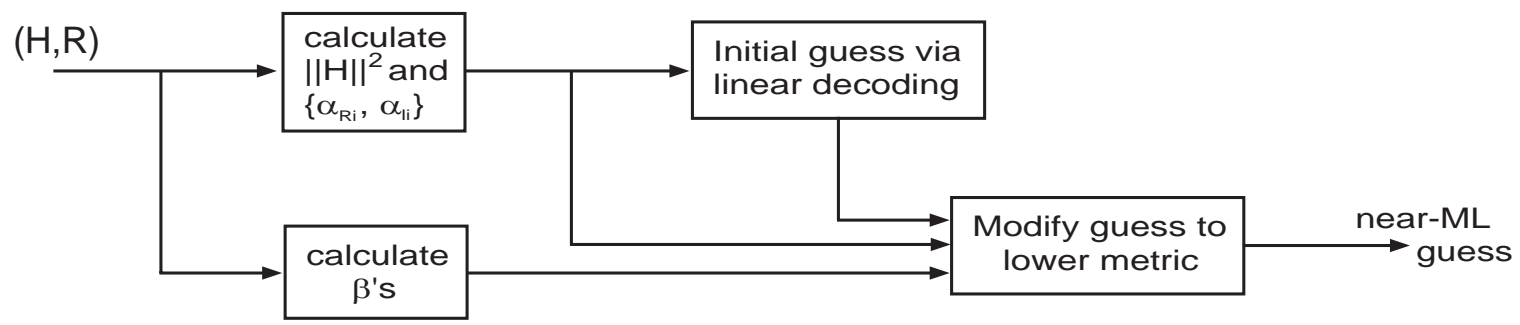

Figure 3.4: A generic framework for a near-ML decoding algorithm for non-orthogonal STBCs.

the SNR and the underlying constellation. This suggests that for large underlying constellations, zero-forcing becomes a good choice.

\subsubsection{A New Framework for Developing Sub-optimal Decoding Algorithms}

Instead of borrowing algorithms used in the sub-optimal decoding of spatial multiplexing systems, let us use (2.4) as a framework to develop a new family of sub-optimal decoding algorithms for non-orthogonal STBCs.

Consider an algorithm based on the scheme shown in Figure 3.4. By calculating $\|\mathbf{H}\|^{2}$ and the $\left\{\alpha_{R i}, \alpha_{I i}\right\}$ values, a simple initial guess is made by independently decoding each symbol using the decoupled terms in (2.4). The closer the STBC is to being orthogonal, the more accurate the initial guess will be. Meanwhile, the contribution of the coupled terms $(\beta$ 's) is calculated, and by modifying the "least reliable" coordinates of the initial guess (in terms of contributing the least to minimizing the metric), neighbours of the initial guess are listed. An exhaustive search is then performed locally to establish which codeword in the list minimizes the metric.

To judge the potential of such a family of decoding algorithms, let us develop 
a simple sub-optimal decoding scheme for the 8 transmit antenna QOSTBC constructed in (2.5) with 16-QAM. The metric can be separated into 4 independent terms: $f_{16}\left(x_{1}, x_{6}\right)+f_{25}\left(x_{2}, x_{5}\right)+f_{38}\left(x_{3}, x_{8}\right)+f_{47}\left(x_{4}, x_{7}\right)$. Simplifying each metric term using (2.4), we can express $f_{16}\left(x_{1}, x_{6}\right)$ as follows:

$$
\begin{aligned}
f_{16}\left(x_{1}, x_{6}\right)= & -\beta_{1}\left(\Re\left\{x_{1}\right\} \Im\left\{x_{1}\right\}+\Re\left\{x_{6}\right\} \Im\left\{x_{6}\right\}\right)+\beta_{2}\left(\Re\left\{x_{1}\right\} \Re\left\{x_{6}\right\}+\Im\left\{x_{1}\right\} \Im\left\{x_{6}\right\}\right) \\
& +\beta_{3}\left(\Re\left\{x_{1}\right\} \Im\left\{x_{6}\right\}+\Im\left\{x_{1}\right\} \Re\left\{x_{6}\right\}\right) \\
& +\|\mathbf{H}\|^{2}\left(\Re\left\{x_{1}\right\}^{2}+\Im\left\{x_{1}\right\}^{2}+\Re\left\{x_{6}\right\}^{2}+\Im\left\{x_{6}\right\}^{2}\right) \\
& +\alpha_{R 1} \Re\left\{x_{1}\right\}+\alpha_{I 1} \Im\left\{x_{1}\right\}+\alpha_{R 6} \Re\left\{x_{6}\right\}+\alpha_{I 6} \Im\left\{x_{6}\right\}
\end{aligned}
$$

where

$$
\begin{aligned}
\beta_{1}= & 4\left(\Re\left\{\left\langle\mathbf{h}_{1}, \mathbf{h}_{3}\right\rangle\right\}+\Re\left\{\left\langle\mathbf{h}_{2}, \mathbf{h}_{4}\right\rangle\right\}+\Re\left\{\left\langle\mathbf{h}_{5}, \mathbf{h}_{7}\right\rangle\right\}+\Re\left\{\left\langle\mathbf{h}_{6}, \mathbf{h}_{8}\right\rangle\right\}\right) \\
\beta_{2}= & 4\left(\Re\left\{\left\langle\mathbf{h}_{1}, \mathbf{h}_{6}\right\rangle\right\}-\Re\left\{\left\langle\mathbf{h}_{2}, \mathbf{h}_{5}\right\rangle\right\}+\Re\left\{\left\langle\mathbf{h}_{3}, \mathbf{h}_{8}\right\rangle\right\}-\Re\left\{\left\langle\mathbf{h}_{4}, \mathbf{h}_{7}\right\rangle\right\}\right) \\
\beta_{3}= & 4\left(-\Re\left\{\left\langle\mathbf{h}_{1}, \mathbf{h}_{8}\right\rangle\right\}+\Re\left\{\left\langle\mathbf{h}_{2}, \mathbf{h}_{7}\right\rangle\right\}-\Re\left\{\left\langle\mathbf{h}_{3}, \mathbf{h}_{6}\right\rangle\right\}+\Re\left\{\left\langle\mathbf{h}_{4}, \mathbf{h}_{5}\right\rangle\right\}\right) \\
\alpha_{R 1}= & \sum_{i=1}^{8} \Re\left\{\left\langle\mathbf{r}_{i}, \mathbf{h}_{i}\right\rangle\right\} \\
\alpha_{I 1}= & -2\left[-\Re\left\{\left\langle\mathbf{r}_{1}, \mathbf{h}_{3}\right\rangle\right\}-\Re\left\{\left\langle\mathbf{r}_{2}, \mathbf{h}_{4}\right\rangle\right\}-\Re\left\{\left\langle\mathbf{r}_{3}, \mathbf{h}_{1}\right\rangle\right\}-\Re\left\{\left\langle\mathbf{r}_{4}, \mathbf{h}_{2}\right\rangle\right\}\right. \\
& \left.-\Re\left\{\left\langle\mathbf{r}_{5}, \mathbf{h}_{7}\right\rangle\right\}-\Re\left\{\left\langle\mathbf{r}_{6}, \mathbf{h}_{8}\right\rangle\right\}-\Re\left\{\left\langle\mathbf{r}_{7}, \mathbf{h}_{5}\right\rangle\right\}-\Re\left\{\left\langle\mathbf{r}_{8}, \mathbf{h}_{6}\right\rangle\right\}\right] \\
\alpha_{R 6}= & -2\left[\Re\left\{\left\langle\mathbf{r}_{1}, \mathbf{h}_{6}\right\rangle\right\}-\Re\left\{\left\langle\mathbf{r}_{2}, \mathbf{h}_{5}\right\rangle\right\}+\Re\left\{\left\langle\mathbf{r}_{3}, \mathbf{h}_{8}\right\rangle\right\}-\Re\left\{\left\langle\mathbf{r}_{4}, \mathbf{h}_{7}\right\rangle\right\}\right. \\
& \left.-\Re\left\{\left\langle\mathbf{r}_{5}, \mathbf{h}_{2}\right\rangle\right\}+\Re\left\{\left\langle\mathbf{r}_{6}, \mathbf{h}_{1}\right\rangle\right\}-\Re\left\{\left\langle\mathbf{r}_{7}, \mathbf{h}_{4}\right\rangle\right\}+\Re\left\{\left\langle\mathbf{r}_{8}, \mathbf{h}_{3}\right\rangle\right\}\right] \\
\alpha_{I 6}= & -2\left[-\Re\left\{\left\langle\mathbf{r}_{1}, \mathbf{h}_{8}\right\rangle\right\}+\Re\left\{\left\langle\mathbf{r}_{2}, \mathbf{h}_{7}\right\rangle\right\}-\Re\left\{\left\langle\mathbf{r}_{3}, \mathbf{h}_{6}\right\rangle\right\}+\Re\left\{\left\langle\mathbf{r}_{4}, \mathbf{h}_{5}\right\rangle\right\}\right. \\
& \left.+\Re\left\{\left\langle\mathbf{r}_{5}, \mathbf{h}_{4}\right\rangle\right\}-\Re\left\{\left\langle\mathbf{r}_{6}, \mathbf{h}_{3}\right\rangle\right\}+\Re\left\{\left\langle\mathbf{r}_{7}, \mathbf{h}_{2}\right\rangle\right\}-\Re\left\{\left\langle\mathbf{r}_{8}, \mathbf{h}_{1}\right\rangle\right\}\right] .
\end{aligned}
$$

The other terms $f_{25}\left(x_{2}, x_{5}\right), f_{38}\left(x_{3}, x_{8}\right)$, and $f_{47}\left(x_{4}, x_{7}\right)$ have a similar form. Consider the following algorithm for decoding the $f_{16}\left(x_{1}, x_{6}\right)$ term:

1. $\mathbf{H}$ and $\mathbf{R}$ are used to calculate $\beta_{1}, \beta_{2}, \beta_{3}, \alpha_{R 1}, \alpha_{I 1}, \alpha_{R 6}, \alpha_{I 6}$, and $\|\mathbf{H}\|^{2}$. 
2. The value $\|\mathbf{H}\|^{2} \Re\left\{x_{1}\right\}^{2}+\alpha_{R 1} \Re\left\{x_{1}\right\}$ is calculated for each value of $\Re\left\{x_{1}\right\} \in$ $\{-3,-1,1,3\}$.

3. The four values calculated in step 2 are listed in order of increasing value. In other words, they are listed in order of "most reliable" to "least reliable," where the most reliable value locally minimizes that decoupled portion of the metric.

4. The $n$ most reliable co-ordinate values are chosen, where $1 \leq n \leq 4$.

5. Steps $2-4$ are repeated for $\Im\left\{x_{1}\right\}, \Re\left\{x_{6}\right\}$, and $\Im\left\{x_{6}\right\}$.

6. A list of $n^{4}$ codeword candidates are generated. An exhaustive search is performed on this list. If $n=4$ then the list will consist of all possible codeword candidates (i.e., the exhaustive search will be optimal). Otherwise, the list will be a subset of all possible codeword candidates.

A pictorial representation is shown in Figure 3.5. Note that this algorithm is similar

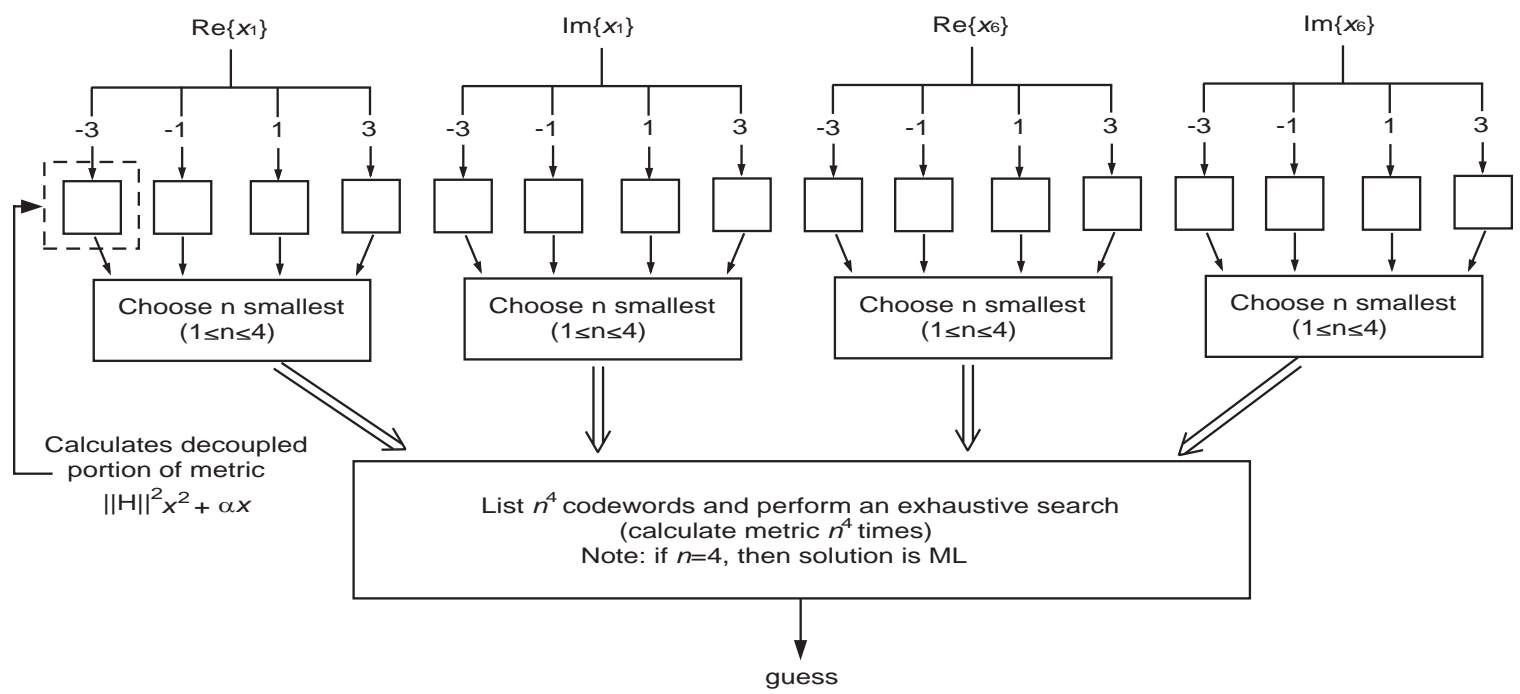

Figure 3.5: An algorithm that uses $n$ to trade-off error performance for a reduction in complexity for the QOSTBC in 2.5 with 16-QAM.

to Chase-decoding-based algorithms [36,37], which perform an exhaustive search on 


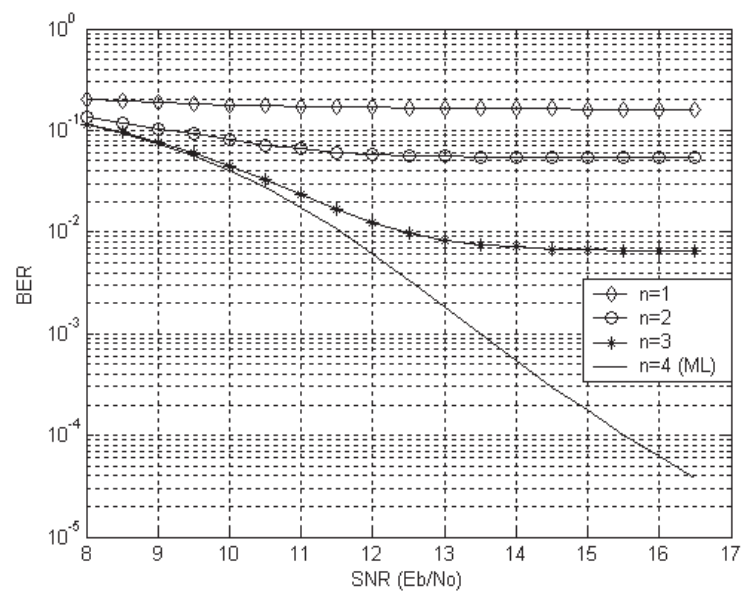

(a) $\mathrm{nR}=1$

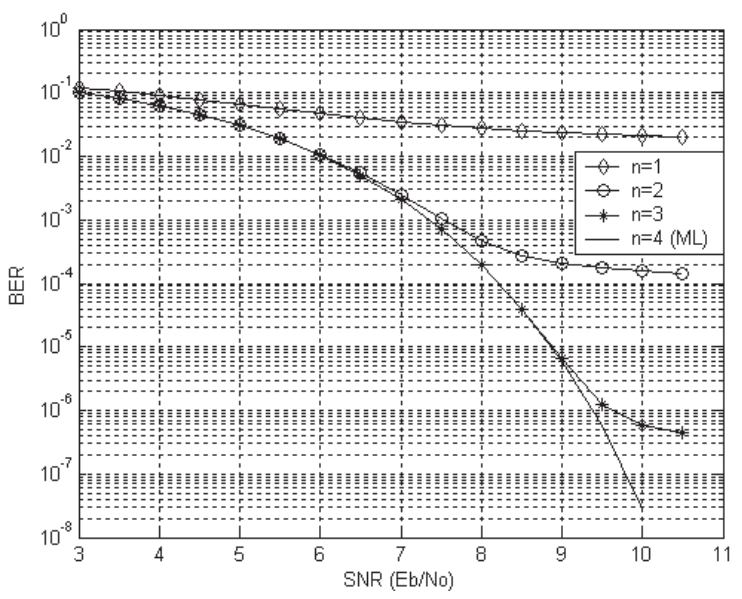

(b) $n R=8$

Figure 3.6: A BER comparison of sub-optimal versus ML for the QOSTBC constructed in 2.5). Rayleigh fading is assumed and the underlying constellation is 16-QAM.

a list of possible codeword candidates. In Chase-decoding-based algorithms, the list is generated by making an initial guess and then including neighbours of the initial guess using reliability information. In the new sub-optimal algorithm proposed above, the initial guess is that which locally minimizes the decoupled portion of the metric. The number of neighbours chosen depends on the value of $n$. In fact, $n$ offers a trade-off between error-performance and complexity. If $n=4$ the algorithm performs ML decoding; if $n=1$ the algorithm performs a simple initial guess that ignores the contribution of the coupled portion of the metric. A similar algorithm can be applied to the $f_{25}\left(x_{2}, x_{5}\right), f_{38}\left(x_{3}, x_{8}\right)$, and $f_{47}\left(x_{4}, x_{7}\right)$ terms.

In Figure 3.6 the error performance is plotted for each $n$ value, for one and eight receive antennas. Rayleigh fading is assumed. From analyzing Figure 3.6, it is clear that the sub-optimal algorithm results in an "error-floor" that is highly dependent on $n$ and $n_{R}$. The complexity (in terms of real multiplications and additions) is shown 
in Table 3.2, For $n_{R}=8$, a complexity reduction of approximately $60 \%$ is achieved by sacrificing only a fraction of a $\mathrm{dB}$ at $10^{-6}$. Depending on the number of receive antennas and error performance required, a sub-optimal algorithm such as the one proposed above is a desirable option.

\begin{tabular}{|c|c|c|c|c|}
\hline & $n=1$ & $n=2$ & $n=3$ & $n=4$ \\
\hline$n_{R}=1$ & $480 / 448$ & $1659 / 1249$ & $6339 / 4369$ & $18747 / 12705$ \\
\hline$n_{R}=8$ & $2384 / 2352$ & $3731 / 3321$ & $8411 / 6441$ & $20819 / 14777$ \\
\hline
\end{tabular}

Table 3.2: A complexity comparison (in terms of real multiplications/additions) for each $n$ value. Rayleigh fading is assumed, and the QOSTBC is that constructed in (2.5) with 16-QAM.

\subsection{Conclusion}

In this chapter the optimal and sub-optimal decoding of QOSTBCs was examined in light of the metric expression in (2.4). Direction was provided as to when to perform an exhaustive search, when to apply the SDA, and when to use sub-optimal decoding algorithms. A new family of sub-optimal decoding algorithms for QOSTBCs was proposed based on the metric expression.

The next chapter summarizes the contribution of the above two chapters and discusses some open problems resulting from the work. 


\section{Chapter 4}

\section{Future Work}

\subsection{Introduction}

In the previous two chapters, the following was accomplished:

1. A new vectorization for a linear space-time block code was proposed that preserves the redundancy in the space-time block code matrix.

2. By expressing the ML metric using the new vectorization (2.3), a new generic representation of the ML metric expression for linear space-time block codes was proposed in 2.4. . One immediate application of (2.4) is the convenient partial decoupling and simplification of the metric for any linear space-time block code.

3. The new metric expression (2.4) can be used as a design tool to help in the construction of new STBCs with low decoding complexity. As an example, a new QOSTBC was proposed in 2.5.

4. A comparison was conducted to answer the following question: for the family 
of QOSTBCs, when is it best to perform an exhaustive search using a simplified and partially decoupled metric expression, and when should an efficient implementation of the sphere decoding algorithm be applied? We showed that the answer depends on a number of different factors, including the number of transmitted symbols, $n_{s}$, the number of receive antennas, $n_{R}$, the rate and diversity of the STBC, and the size of the underlying constellation. Determining this boundary is an important and practical issue not yet directly addressed in the literature.

5. The new metric expression (2.4) can be used as the framework for a new family of sub-optimal decoding algorithms for STBCs that trade-off error performance for a reduction in decoding complexity. A practical example of such an algorithm was detailed in Section 3.3.3. The algorithm was considered in light of the complexity versus error performance trade-off of other optimal and sub-optimal decoding schemes.

The contributions listed above have resulted in the following published and submitted works: [38-42].

Upon analyzing the above work, some immediate questions arise. For example, how can one use the "coupled symbols" term in (2.4) to directly construct STBCs guaranteed to have low decoding complexity? Also, what will the complexity versus error performance trade-off be like for the new sub-optimal decoding algorithm in Section 3.3 .3 applied to other QOSTBCs? These questions merit a future investigation.

The remainder of this chapter focuses in detail on the challenges of trying to answer two open problems resulting from the contributions above. In Section 4.2 , 
the challenge of trying to analytically justify the optimum rotation angle for the new QOSTBC proposed in 2.5 is considered. In Section 4.3 , the challenge of using the redundancy in the new vectorization scheme to develop an optimality test criterion for STBCs is considered. The thesis is concluded in Section 4.4.

\subsection{Optimum Rotation Angle for the New QOSTBC}

In 2.5) a new rate-one QOSTBC with 8 antennas is proposed with lower decoding complexity than that in [11]. Using a computer to perform a numerical search, it can be shown that an appropriate constellation rotation exists to make (2.5) full-diversity. For 4-QAM this angle is found to be $\pi / 8$ (see Figure 2.1). The problem is to attempt to justify this rotation angle analytically.

In this section, rotations for QOSTBCs will be reviewed, along with a full literature review. The challenge of analytically justifying the $\pi / 8$ rotation angle will then be discussed in light of previous publications in the area.

\subsubsection{Background: constellation rotation}

For any two distinct $n_{T} \times L$ codeword matrices, $\mathbf{C}^{\mathbf{i}}$ and $\mathbf{C}^{\mathbf{j}}(i \neq j)$, the codeword distance matrix is defined as

$$
A\left(\mathbf{C}^{\mathbf{i}}, \mathbf{C}^{\mathbf{j}}\right)=\left(\mathbf{C}^{\mathbf{j}}-\mathbf{C}^{\mathbf{i}}\right)\left(\mathbf{C}^{\mathbf{j}}-\mathbf{C}^{\mathbf{i}}\right)^{H} .
$$

Through pairwise error probability analysis, it can be shown that the diversity of a STBC is equal to the minimum rank of $A\left(\mathbf{C}^{\mathbf{i}}, \mathbf{C}^{\mathbf{j}}\right)$ over all $i \neq j$ [5, chap. 3].

For $\mathbf{C}^{\mathbf{i}}$ and $\mathbf{C}^{\mathbf{j}}$, the coding gain distance (CGD) is defined as

$$
C G D\left(\mathbf{C}^{\mathbf{i}}, \mathbf{C}^{\mathbf{j}}\right)=\operatorname{det}\left(A\left(\mathbf{C}^{\mathbf{i}}, \mathbf{C}^{\mathbf{j}}\right)\right) .
$$


$A\left(\mathbf{C}^{\mathbf{i}}, \mathbf{C}^{\mathbf{j}}\right)$ is full rank if and only if $C G D\left(\mathbf{C}^{\mathbf{i}}, \mathbf{C}^{\mathbf{j}}\right) \neq 0$. In general, a given QOSTBC is not full diversity since two distinct codeword matrices, $\mathbf{C}^{\mathbf{i}}$ and $\mathbf{C}^{\mathbf{j}}(i \neq j)$ can be chosen such that $C G D\left(\mathbf{C}^{\mathbf{i}}, \mathbf{C}^{\mathbf{j}}\right)=0$. However, by choosing some of the symbols from a constellation rotated by angle $\theta$, we can avoid this problem and achieve full-diversity. Specifically, we must choose an underlying constellation rotation that ensures for all $(i, j)$

$$
\min _{i \neq j} C G D\left(\mathbf{C}^{\mathbf{i}}, \mathbf{C}^{\mathbf{j}}\right) \neq 0 .
$$

Furthermore, in order to maximize the coding gain, we would like to maximize $\min _{i \neq j} C G D\left(\mathbf{C}^{\mathbf{i}}, \mathbf{C}^{\mathbf{j}}\right)$. Whereas an infinite number of constellation rotation angles will satisfy (4.3), only the optimum constellation rotation angle, $\theta_{\text {opt }}$, will both satisfy (4.3) and maximize the value of $\min _{i \neq j} C G D\left(\mathbf{C}^{\mathbf{i}}, \mathbf{C}^{\mathbf{j}}\right)$.

Note that an equivalent form of (4.3) is the "diversity product" [14,43,44]:

$$
\zeta=\frac{1}{2 \sqrt{n_{T}}} \min _{i \neq j}\left[C G D\left(\mathbf{C}^{\mathbf{i}}, \mathbf{C}^{\mathbf{j}}\right)\right]^{\frac{1}{2 L}} .
$$

\subsubsection{QOSTBCs with no co-ordinate interleaving}

\section{Transmit Antennas}

Consider a $n_{T}=L=4$ QOSTBC, such as that proposed in [10]. The metric for this QOSTBC can be separated into two non-interacting terms: $f_{14}\left(x_{1}, x_{4}\right)+f_{23}\left(x_{2}, x_{3}\right)$. To achieve full-diversity, some sort of rotation scheme needs to be applied. In [14] it is proposed that $x_{1}, x_{2}$ be taken from underlying constellation $\mathcal{A}$, and $x_{3}, x_{4}$ be taken from a rotated constellation $e^{j \theta} \mathcal{A}$, i.e., $x_{k} \rightarrow \hat{x_{k}}=e^{j \theta} x_{k}, k=3$, 4 . To determine an optimum value of $\theta$, the following steps are taken:

1. first an explicit expression is found for the diversity product (4.4) in terms of $x_{1}$, 
$x_{2}, \hat{x_{3}}$, and $\hat{x_{4}}[14$, eqn. (22)];

2. it is then shown that the diversity product expression can be related to the minimum distance between constellations $\mathcal{A}$ and $e^{j \theta} \mathcal{A}$, which is upper-bounded by the minimum distance of constellation $\mathcal{A}$;

3. for QAM, it is then argued that this upper-bound is met with equality and occurs when $\theta=\pi / 4$. Therefore, $\theta=\theta_{\text {opt }}=\pi / 4$ maximizes 4.4 .

\section{Comments On Related Papers}

[13] is a work published before [14]. It does not have an analytical approach as rigorous as [14] (in fact, optimum rotation angles are found through computer simulation). However, it is the first paper to suggest simplifying analysis by zeroing out groups of symbols in the STBC matrix that do not interact with other symbols. For example, divide the $n_{T}=L=4$ QOSTBC in [10] as follows: $G\left(x_{1}, x_{2}, x_{3}, x_{4}\right)=$ $G\left(x_{1}, 0,0, x_{4}\right)+G\left(0, x_{2}, x_{3}, 0\right)$. We can then just consider $G\left(x_{1}, 0,0, x_{4}\right)$ and find the optimum rotation angle for the constellation $x_{4}$ is drawn from. $x_{3}$ will be drawn from the same constellation as $x_{4}$ due to the non-interacting metric expression $f_{14}\left(x_{1}, x_{4}\right)+f_{23}\left(x_{2}, x_{3}\right)$.

[43] builds on [14] (and others, such as $[45,46]$ ), by proposing optimal rotation angles for MPSK constellations, where M may be even or odd. The derivation in [43] parallels that in [14]. Specifically, (4.4) is expressed in terms of the symbols mapped to the QOSTBC, and then deductions and arguments are made based on 4.4.

[47] independently derives the same results as [43], but uses a geometric argument to maximize 4.4. This geometric method is particular to MPSK constellations. 


\section{Transmit Antennas}

The approach to finding $\theta_{\text {opt }}$ in [14] holds for QOSTBCs (without co-ordinate interleaving) whose metric can be separated into non-interacting groups of two symbols. This includes QOSTBCs for $n_{T}>4$ (but rate less than one), constructed by applying the modular/recursive technique in [10] to orthogonal designs in [9].

The only rate-one full-diversity $n_{T}=8 \mathrm{QOSTBC}$ proposed in the literature is that in [11]. Its metric decouples into two non-interacting terms: $f_{1278}\left(x_{1}, x_{2}, x_{7}, x_{8}\right)+$ $f_{3456}\left(x_{3}, x_{4}, x_{5}, x_{6}\right)$. Let us zero out $x_{3}, x_{4}, x_{5}$, and $x_{6}$ as suggested in [13] and consider only the $f_{1278}\left(x_{1}, x_{2}, x_{7}, x_{8}\right)$ term. To achieve full-diversity we need 3 rotated underlying constellations: $x_{1} \in \mathcal{A}, x_{2} \in e^{j \theta_{1}} \mathcal{A}, x_{7} \in e^{j \theta_{2}} \mathcal{A}$, and $x_{8} \in e^{j \theta_{3}} \mathcal{A}$. Optimizing over more than one angle becomes very difficult analytically. Therefore, [11] uses the following approach that combines (mostly) computer simulation with some analytical steps:

1. first it is shown using computer simulation that $\theta_{3}=\theta_{1}+\theta_{2}$, where $\theta_{2}$ is unknown and $\theta_{1}$ is $\pi / 4$ for QAM and $\pi / M$ for MPSK (see [11, eqn. (7)]);

2. $\theta_{2}$ is then found analytically for 4-QAM. It is found using computer simulations for all higher level constellations.

For 4-QAM, $\theta_{2}=\pi / 6$ (or $\pi / 12$ ), $\theta_{1}=\pi / 4$, and therefore $\theta_{3}=\pi / 4+\pi / 6=5 \pi / 12$. Figure 2.1(a) plots the SER when the above constellation rotations are applied.

\subsubsection{Rotations for QOSTBCs with co-ordinate interleaving}

In $[29,48,49]$ STBCs from co-ordinate interleaved orthogonal designs (CIODs) are introduced and discussed. These STBCs are orthogonal, i.e., the metric completely decouples into non-interacting terms. However, unlike OSTBCs designed in [9], they 
are not full-diversity for any arbitrary underlying constellation. By analyzing the CGD for the family of CIODs, it is shown in [49] that (4.3) will not be satisfied if the underlying constellation has signal points with equal co-ordinates. For example, in the 4-QAM constellation, the points $1+j$ and $1-j$ have the same real co-ordinate, therefore (4.3) will not be satisfied, and the CIOD will not be full-diversity. This can be alleviated by simply drawing all symbols from a rotated constellation. The optimum rotation angle, $\theta_{\text {opt }}$, will both satisfy (4.3) and maximize the CGD. In [49, Appendix], $\theta_{\text {opt }}$ is derived analytically using an approach paralleling that in [14]. Specifically, the CGD is evaluated and expressed in terms of the underlying constellation symbols and rotation angle $\theta$. By analyzing this expression, an equation can be set up to solve for $\theta_{\text {opt }}$. This analytical derivation is straightforward since for CIODs the CGD is a simple expression.

In [12], QOSTBCs are proposed that use co-ordinate interleaving. These QOSTBCs suffer from the same problem as CIODs: the underlying constellation needs to be rotated to achieve full-diversity. In [44], an analysis that parallels [49, Appendix] is performed to derive $\theta_{\text {opt }}$ for the family of MDC-QOSTBCs. This derivation is also relatively straightforward, since for the family of MDC-QOSTBCs, the expression for the CGD is just as simple as the CIOD case. It is shown in [44] that for 4-QAM, $\theta_{\text {opt }}=13.28^{\circ}$.

Important Note: In the STBCs above based on co-ordinate interleaving, all symbols are taken from the same rotated constellation. This is different from the QOSTBCs in Section 4.2.2, where half of the symbols are taken from a non-rotated constellation, and the other half are taken from a rotated constellation. 


\subsubsection{Challenges of Deriving the Optimum Rotation Angle for the new QOSTBC}

The new QOSTBC in (2.5) uses co-ordinate interleaving, but unlike the STBCs in Section 4.2.3, the simplified metric expression results in the coupling of multiple symbols. This poses the following challenges:

1. Based on the discussion above, it would seem that in order for (2.5) to achieve full-diversity, all symbols should be taken from rotated constellations. Specifically, $\left(x_{1}, x_{2}, x_{3}, x_{4}\right)$ should be taken from a constellation rotated by $\theta_{1}$ (to satisfy the rotation necessary from interleaving), and $\left(x_{5}, x_{6}, x_{7}, x_{8}\right)$ should be taken from a constellation rotated by $\theta_{2}$ (to satisfy both the rotation necessary for interleaving and the rotation necessary relative to $\left.\left(x_{1}, x_{2}, x_{3}, x_{4}\right)\right)$. $\theta_{1}$ and $\theta_{2}$ would need to be jointly optimized such that both 4.3 is satisfied and $\min _{i \neq j} C G D\left(\mathbf{C}^{\mathbf{i}}, \mathbf{C}^{\mathbf{j}}\right)$ is maximized. Yet, based on Figure 2.1(b), two rotation angles are not necessary.

2. All previous approaches in the literature use the explicit CGD expression 4.2) to derive a rotation angle that maximizes $\min _{i \neq j} C G D\left(\mathbf{C}^{\mathbf{i}}, \mathbf{C}^{\mathbf{j}}\right){ }^{1}$. The CGD is relatively simple for the previous cases (e.g. [5, eqn. (5.10)], [14, eqn. (22)], [49], [44, eqn. (12)]), but for the new QOSTBC construced in 2.5), this is not the case. For example, consider zeroing out all terms except $\left(x_{1}, x_{6}\right)$. Therefore, (2.5) reduces to

\footnotetext{
${ }^{1}$ Even [47], which offers a geometric approach, uses the explicit CGD expression as a basis for the scheme.
} 


$$
\mathbf{C}=\left[\begin{array}{cccccccc}
\Re\left\{x_{1}\right\} & 0 & -\Im\left\{x_{1}\right\} & 0 & 0 & \Re\left\{x_{6}\right\} & 0 & -\Im\left\{x_{6}\right\} \\
0 & \Re\left\{x_{1}\right\} & 0 & -\Im\left\{x_{1}\right\} & -\Re\left\{x_{6}\right\} & 0 & \Im\left\{x_{6}\right\} & 0 \\
-\Im\left\{x_{1}\right\} & 0 & \Re\left\{x_{1}\right\} & 0 & 0 & -\Im\left\{x_{6}\right\} & 0 & \Re\left\{x_{6}\right\} \\
0 & -\Im\left\{x_{1}\right\} & 0 & \Re\left\{x_{1}\right\} & \Im\left\{x_{6}\right\} & 0 & -\Re\left\{x_{6}\right\} & 0 \\
0 & -\Re\left\{x_{6}\right\} & 0 & \Im\left\{x_{6}\right\} & \Re\left\{x_{1}\right\} & 0 & -\Im\left\{x_{1}\right\} & 0 \\
\Re\left\{x_{6}\right\} & 0 & -\Im\left\{x_{6}\right\} & 0 & 0 & \Re\left\{x_{1}\right\} & 0 & -\Im\left\{x_{1}\right\} \\
0 & \Im\left\{x_{6}\right\} & 0 & -\Re\left\{x_{6}\right\} & -\Im\left\{x_{1}\right\} & 0 & \Re\left\{x_{1}\right\} & 0 \\
-\Im\left\{x_{6}\right\} & 0 & \Re\left\{x_{6}\right\} & 0 & 0 & -\Im\left\{x_{1}\right\} & 0 & \Re\left\{x_{1}\right\}
\end{array}\right] .
$$

For any two codewords, $\mathbf{C}^{\mathbf{i}}$ and $\mathbf{C}^{\mathbf{j}}$, the coding gain distance 4.2 can be simplified to

$$
\begin{aligned}
C G D\left(\mathbf{C}^{\mathbf{i}}, \mathbf{C}^{\mathbf{j}}\right) & =\operatorname{det}\left(\left[\begin{array}{cc}
A & B \\
-B & A
\end{array}\right]\right) \\
& =\operatorname{det}(A) \operatorname{det}\left(A+B A^{-1} B\right)
\end{aligned}
$$

where

$$
\begin{gathered}
A=\left[\begin{array}{llll}
e_{1} & 0 & e_{2} & 0 \\
0 & e_{1} & 0 & e_{2} \\
e_{2} & 0 & e_{1} & 0 \\
0 & e_{2} & 0 & e_{1}
\end{array}\right], B=\left[\begin{array}{cccc}
0 & e_{3} & 0 & e_{4} \\
-e_{3} & 0 & -e_{4} & 0 \\
0 & e_{4} & 0 & e_{3} \\
-e_{4} & 0 & -e_{3} & 0
\end{array}\right], \\
e_{1}=\left(\Re\left\{x_{1}^{(i)}\right\}-\Re\left\{x_{1}^{(j)}\right\}\right)^{2}+\left(\Im\left\{x_{1}^{(i)}\right\}-\Im\left\{x_{1}^{(j)}\right\}\right)^{2}+\left(\Re\left\{x_{6}^{(i)}\right\}-\Re\left\{x_{6}^{(j)}\right\}\right)^{2}+\left(\Im\left\{x_{6}^{(i)}\right\}-\Im\left\{x_{6}^{(j)}\right\}\right)^{2}, \\
e_{2}=-2\left[\left(\Re\left\{x_{1}^{(i)}\right\}-\Re\left\{x_{1}^{(j)}\right\}\right)\left(\Im\left\{x_{1}^{(i)}\right\}-\Im\left\{x_{1}^{(j)}\right\}\right)+\left(\Re\left\{x_{6}^{(i)}\right\}-\Re\left\{x_{6}^{(j)}\right\}\right)\left(\Im\left\{x_{6}^{(i)}\right\}-\Im\left\{x_{6}^{(j)}\right\}\right)\right], \\
e_{3}=2\left[\left(\Re\left\{x_{1}^{(i)}\right\}-\Re\left\{x_{1}^{(j)}\right\}\right)\left(\Re\left\{x_{6}^{(i)}\right\}-\Re\left\{x_{6}^{(j)}\right\}\right)+\left(\Im\left\{x_{1}^{(i)}\right\}-\Im\left\{x_{1}^{(j)}\right\}\right)\left(\Im\left\{x_{6}^{(i)}\right\}-\Im\left\{x_{6}^{(j)}\right\}\right)\right], \\
e_{4}=-2\left[\left(\Re\left\{x_{1}^{(i)}\right\}-\Re\left\{x_{1}^{(j)}\right\}\right)\left(\Im\left\{x_{6}^{(i)}\right\}-\Im\left\{x_{6}^{(j)}\right\}\right)+\left(\Im\left\{x_{1}^{(i)}\right\}-\Im\left\{x_{1}^{(j)}\right\}\right)\left(\Re\left\{x_{6}^{(i)}\right\}-\Re\left\{x_{6}^{(j)}\right\}\right)\right] .
\end{gathered}
$$


Expressing (4.6) in terms of $\Re\left\{x_{1}^{(i)}\right\}, \Re\left\{x_{1}^{(j)}\right\}, \Re\left\{x_{6}^{(i)}\right\}, \Re\left\{x_{6}^{(j)}\right\}, \Im\left\{x_{1}^{(i)}\right\}, \Im\left\{x_{1}^{(j)}\right\}$, $\Im\left\{x_{6}^{(i)}\right\}$, and $\Im\left\{x_{6}^{(j)}\right\}$ is very complicated, and will result in an expression that is much more complex than the $4 \times 4$ case (such as that in [44, eqn. (12)] or [5, eqn. (5.10) or (5.16)]). Even if (4.6) was evaluated, it would be very difficult to analytically justify that $\pi / 8$ not only satisfies (4.3) (as an infinite number of other rotation angles would), but that $\pi / 8$ maximizes (4.3) over all other rotation angles. Furthermore, it's not clear whether (4.6) would evaluate differently for other pairs of symbols. For example, consider zeroing out all terms except $\left(x_{4}, x_{7}\right)$. Therefore, 2.5 reduces to

$\mathbf{C}=\left[\begin{array}{cccccccc}0 & j \Re\left\{x_{4}\right\} & 0 & j \Im\left\{x_{4}\right\} & j \Re\left\{x_{7}\right\} & 0 & j \Im\left\{x_{7}\right\} & 0 \\ j \Re\left\{x_{4}\right\} & 0 & j \Im\left\{x_{4}\right\} & 0 & 0 & -j \Re\left\{x_{7}\right\} & 0 & -j \Im\left\{x_{7}\right\} \\ 0 & j \Im\left\{x_{4}\right\} & 0 & j \Re\left\{x_{4}\right\} & j \Im\left\{x_{7}\right\} & 0 & j \Re\left\{x_{7}\right\} & 0 \\ j \Im\left\{x_{4}\right\} & 0 & j \Re\left\{x_{4}\right\} & 0 & 0 & -j \Im\left\{x_{7}\right\} & 0 & -j \Re\left\{x_{7}\right\} \\ j \Re\left\{x_{7}\right\} & 0 & j \Im\left\{x_{7}\right\} & 0 & 0 & -j \Re\left\{x_{4}\right\} & 0 & -j \Im\left\{x_{4}\right\} \\ 0 & -j \Re\left\{x_{7}\right\} & 0 & -j \Im\left\{x_{7}\right\} & -j \Re\left\{x_{4}\right\} & 0 & -j \Im\left\{x_{4}\right\} & 0 \\ j \Im\left\{x_{7}\right\} & 0 & j \Re\left\{x_{7}\right\} & 0 & 0 & -j \Im\left\{x_{4}\right\} & 0 & -j \Re\left\{x_{4}\right\} \\ 0 & -j \Im\left\{x_{7}\right\} & 0 & -j \Re\left\{x_{7}\right\} & -j \Im\left\{x_{4}\right\} & 0 & -j \Re\left\{x_{4}\right\} & 0\end{array}\right]$.

This is a different form than for the pair of symbols $\left(x_{1}, x_{6}\right)$. It is not clear if 4.2 would be evaluated to 4.6 .

\subsubsection{Conclusions}

As discussed above, analytically justifying the optimal rotation angle for the new QOSTBC in (2.5) is challenging. A future investigation is needed that involves either coming up with a geometric justification, or analyzing the CGD 4.2 in more detail. 


\subsection{Development of Optimality Test Criteria For STBCs}

One of the motivating applications for the new vectorization proposed in $(2.3)$ is the development of optimality test criteria (OTC) for linear STBCs. This is because the development of OTC requires a concept of redundancy in a STBC, which the new vectorization provides.

OTC are also sometimes called "codeword tests" or "acceptance criteria"; these three terms will be used interchangeably. In short, OTC are low-complexity tests to check if a given codeword under consideration is the ML solution. In decoding algebraic codes communicated through AWGN, OTC are used to terminate the decoding algorithm early in schemes such as Chase decoding. An overview of the subject can be found in [50, Chap. 10] and [51], as well as in the material below.

In the following sections, the derivation of a codeword test for an algebraic code transmitted through AWGN will be reviewed. The OTC developed will be equivalent to that in [52], although the notation and approach used will be that in [37]. The optimality test criterion will then be extended to a SISO system in a fading environment. The challenges of generalizing to a MIMO system with space-time block coding will then be discussed. It will be shown that the new vectorization and metric expression provide a promising framework for developing OTC for STBCs in MIMO systems, but that there are still challenges to overcome. 


\subsubsection{An Optimality Test Criterion For A SISO System}

First, consider the classic case of transmitting an $[n, k, d]$ algebraic code through a single-input single-output (SISO) system with only AWGN. Assume the underlying constellation is BPSK. At the receiver, the transmitted codeword $\mathbf{r}=\mathbf{x}+\mathbf{n}$ is received where $\mathbf{r}=\left(\begin{array}{llll}r_{1} & r_{2} & \ldots & r_{n}\end{array}\right), \quad r_{i}=x_{i}+n_{i}, x_{i} \in\{-1,1\}^{n}$, and $n_{i} \sim N\left(0, \sigma^{2}\right)$. The ML metric is as follows:

$$
\hat{\mathbf{x}}=\arg \min _{\mathbf{x} \in C}\|\mathbf{r}-\mathbf{x}\|^{2}=\arg \min _{\mathbf{x} \in C}\left\{\|\mathbf{r}\|^{2}+\|\mathbf{x}\|^{2}-2 \mathbf{r}^{T} \mathbf{x}\right\}
$$

which reduces to:

$$
\hat{\mathbf{x}}=\arg \min _{\mathbf{x} \in C}\left\{\|\mathbf{x}\|^{2}-2 \mathbf{r}^{T} \mathbf{x}\right\}=\arg \min _{\mathbf{x} \in C}\left\{\|\mathbf{x}\|^{2}-2\left(\sum_{i=1}^{n}\left|r_{i}\right|-2 \sum_{i: r_{i} x_{i}<0}\left|r_{i}\right|\right)\right\} .
$$

$\|\mathbf{x}\|$ and $\sum_{i=1}^{n}\left|r_{i}\right|$ are independent of the codeword considered, and therefore the metric reduces to minimizing a single term. The most likely codeword, $\hat{\mathbf{x}}$, is the codeword that minimizes the correlation discrepancy $\ell(\mathbf{r}, \mathbf{x})$ :

$$
\hat{\mathbf{x}}=\arg \min _{\mathbf{x} \in C}\{\ell(\mathbf{r}, \mathbf{x})\}
$$

where

$$
\ell(\mathbf{r}, \mathbf{x}) \triangleq \sum_{i: r_{i} x_{i}<0}\left|r_{i}\right| .
$$

Note that each $\left|r_{i}\right|$ value can be interpreted as a measure of how "reliable" the guess $x_{i}$ is. For example, if $r_{i}=-23.5$, a guess of $x_{i}=-1$ is very reliable, but if $r_{i}=-0.3$, then a guess of $x_{i}=-1$ is not very reliable since the received value is close to the decision boundary. Choosing a codeword that minimizes the correlation discrepancy means choosing one that minimizes the sum of reliability values over the codeword coordinates, $x_{i}$, that are in different decision regions than $r_{i}$. 


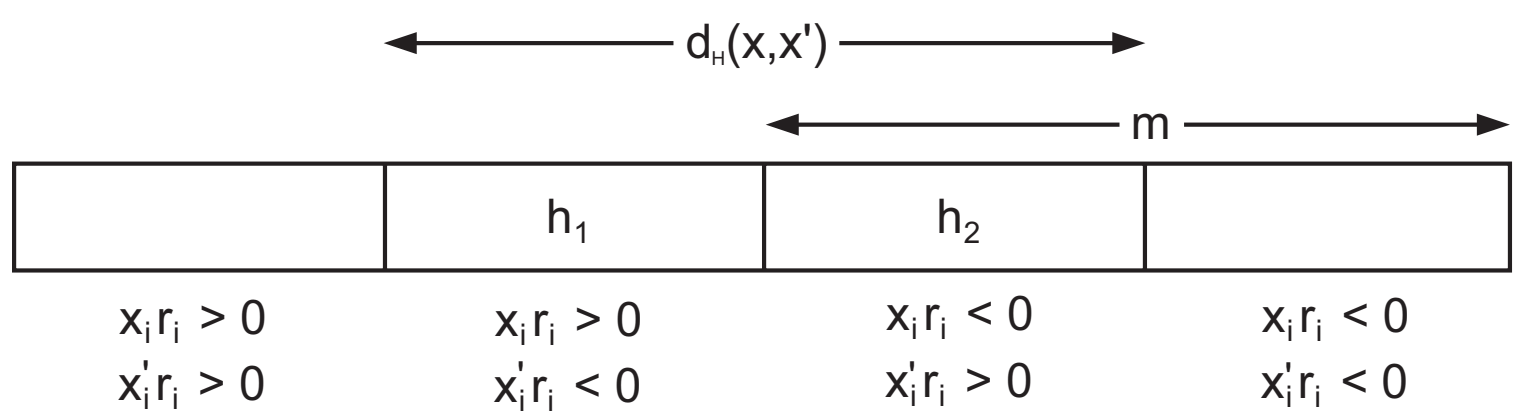

Figure 4.1: Partition of codeword positions.

The approach to derive the codeword test is as follows: find a lower bound on the correlation discrepancy for every other codeword in the code book $\mathbf{x}^{\prime} \neq \mathbf{x}$, where $\mathbf{x}$ is the codeword under consideration. This bound is derived using information from the codeword $\mathbf{x}$. Then, if the correlation discrepancy for $\mathbf{x}$ is less than the derived lower bound, it must be the most likely codeword in the code book. Formally, this is done as follows: let $S_{x}=\left\{i \mid r_{i} x_{i} \geq 0\right\}, S_{x}^{c}=\left\{i \mid r_{i} x_{i}<0\right\}$, and $m=\left|S_{x}^{c}\right|$. Therefore $\ell(\mathbf{r}, \mathbf{x})=\sum_{i \in S_{x}^{c}}\left|r_{i}\right| . \quad \forall \mathbf{x}^{\prime} \in C$ where $\mathbf{x}^{\prime} \neq \mathbf{x}$ let $h_{1}=\left|S_{x^{\prime}}^{c} \cap S_{x}\right|$ and $h_{2}=\left|S_{x^{\prime}} \cap S_{x}^{c}\right|$. Therefore,

$$
\ell\left(\mathbf{r}, \mathbf{x}^{\prime}\right)=\sum_{i \in S_{x^{\prime}}}\left|r_{i}\right| \geq \sum_{\substack{i \in S_{x^{c},}^{\prime}, S_{x} \\ x^{\prime}}}\left|r_{i}\right| \geq \sum_{i=1}^{h_{1}}\left|r_{S_{x}^{(i)}}\right|
$$

where $\left\{\left|r_{S_{x}^{(i)}}\right|\right\}$ represents the set of received vectors in $S_{x}$ reordered in terms of increasing magnitude, i.e., $\left|r_{S_{x}^{(1)}}\right| \leq\left|r_{S_{x}^{(2)}}\right| \leq \ldots$. To continue, a lower bound on $h_{1}$ must be derived. Consider a partition of the codeword positions as shown in Figure 4.1. Using this pictorial representation the following inequalities can easily be derived:

(1) $d_{H}\left(\mathbf{x}, \mathbf{x}^{\prime}\right)=h_{1}+h_{2} \geq d$

(2) $d \leq d_{H}\left(\mathbf{x}, \mathbf{x}^{\prime}\right) \leq 2 m+h_{1}-h_{2}$

Combining the above inequalities to eliminate $h_{2}$ yields $h_{1} \geq d-m$. Therefore, a 
codeword, $\mathbf{x}$, is the most-likely codeword if

$$
\ell(\mathbf{r}, \mathbf{x}) \leq \sum_{i=1}^{d-m}\left|r_{S_{x}^{(i)}}\right| .
$$

This is the Taipale and Pursley codeword test criterion derived in [52]. Note that the criterion depends on the reliability values of the coordinates of the codeword under consideration. If the sum of the $d-m$ smallest reliability values in $S_{x}$ are below a certain threshold, the guess $\mathbf{x}$ is deemed "reliable enough" to be the ML solution. The threshold is determined using the minimum distance, $d$, of the linear block code. Clearly, deriving OTC requires concepts of redundancy and reliability.

As a final note, failure to satisfy the codeword test does not imply the codeword is not the most likely. Also, the criterion is only valid for codewords where $m<d$. In [37] a similar acceptance criterion is also derived for the $q$-ary case. The derivations in [37] assume the decoder has 2 codewords. We only consider one codeword, hence the criterion in [52] is of interest.

\section{An Extension To A Fading Environment}

The codeword test developed above is valid for a SISO system with an underlying BPSK constellation and an outer linear binary block $[n, k, d]$ code. The channel is assumed to have no fading. Before analyzing a MIMO system with a STBC, consider the simple system above in a frequency-flat and fast fading environment. An acceptance criterion can be developed using the same approach:

The transmitted codeword $\mathbf{r}=\mathbf{y}+\mathbf{n}$ is received where $\mathbf{r}=\left(\begin{array}{llll}r_{1} & r_{2} & \ldots & r_{n}\end{array}\right), r_{i}=$ $\Re\left\{r_{i}\right\}+j \Im\left\{r_{i}\right\}, \mathbf{y}=\left(y_{1} y_{2} \ldots y_{n}\right), y_{i}=\left(\Re\left\{h_{i}\right\}+j \Im\left\{h_{i}\right\}\right) x_{i}$, and $\mathbf{n}=\left(n_{1} n_{2} \ldots n_{n}\right)$,

$n_{i}=\Re\left\{n_{i}\right\}+j \Im\left\{n_{i}\right\} . x_{i} \in\{-1,1\}^{n}, \Re\left\{n_{i}\right\} \sim N\left(0, \frac{\sigma^{2}}{2}\right), \Im\left\{n_{i}\right\} \sim N\left(0, \frac{\sigma^{2}}{2}\right), \Re\left\{h_{i}\right\} \sim$ 
$N\left(0, \frac{\Omega}{2}\right)$, and $\Im\left\{h_{i}\right\} \sim N\left(0, \frac{\Omega}{2}\right)$. The ML metric is as follows:

$$
\hat{\mathbf{x}}=\arg \min _{\mathbf{x} \in C}\left\{\|\mathbf{y}\|^{2}-2 \Re\left\{\mathbf{r}^{H} \mathbf{y}\right\}\right\}
$$

where

$$
\begin{gathered}
\|\mathbf{y}\|^{2}=\sum_{i=1}^{n}\left(\Re\left\{h_{i}^{2}\right\} x_{i}^{2}+\Im\left\{h_{i}^{2}\right\} x_{i}^{2}\right)=\sum_{i=1}^{n}\left(\Re\left\{h_{i}^{2}\right\}+\Im\left\{h_{i}^{2}\right\}\right) \\
\Re\left\{\mathbf{r}^{H} \mathbf{y}\right\}=\sum_{i=1}^{n} \Re\left\{r_{i}^{*} h_{i}\right\} x_{i} .
\end{gathered}
$$

Therefore,

$$
\hat{\mathbf{x}}=\arg \max _{\mathbf{x} \in C}\left\{\sum_{i=1}^{n} \Re\left\{r_{i}^{*} h_{i}\right\} x_{i}\right\}
$$

which reduces to minimizing a correlation discrepancy measure:

$$
\hat{\mathbf{x}}=\arg \min _{\mathbf{x} \in C}\left\{\sum_{i: \Re\left\{r_{i}^{*} h_{i}\right\} x_{i}<0}\left|\Re\left\{r_{i}^{*} h_{i}\right\}\right|\right\}
$$

The metric in (4.10) is of the same form as the metric in (4.7), except that the reliability measure of each codeword coordinate, $x_{i}$, is $\left|\Re\left\{r_{i}^{*} h_{i}\right\}\right|$ instead of $\left|r_{i}\right|$. Therefore, an acceptance criterion can be developed via the same derivation. The criterion is as follows: a codeword, $\mathbf{x}$, is the most-likely codeword if

$$
\ell(\mathbf{r}, \mathbf{x}) \leq \sum_{i=1}^{d-m}\left|\Re\left\{r_{S_{x}}^{*} h_{S_{x}}^{(i)}\right\}\right|
$$

where $m=\left\{i \mid \Re\left\{r_{i}^{*} h_{i}\right\} x_{i}<0\right\}$. (4.11) differs from (4.8) only by the inclusion of the fading information.

\subsubsection{OTC for a MIMO System with STBCs}

As shown above, it is relatively easy to extend the classic Taipale and Pursley codeword test [52] to a SISO system with fading. The transmission of a STBC over a 
MIMO channel is a much more complex problem; however, a promising approach is to parallel the above development. In the SISO fading case in Section 4.3.1 we used an outer code with redundancy $d$ and determined the codeword transmitted is most likely if its associated reliability information was below a certain threshold, i.e, if (4.11) was satisfied. In the MIMO analysis, we are not assuming an outer code, but the STBC has inherent redundancy in it that can be exploited. For example, consider the Alamouti code [3]:

$$
\begin{aligned}
\mathbf{C} & =\left[\begin{array}{ll}
c_{11} & c_{12} \\
c_{21} & c_{22}
\end{array}\right] \\
& =\left[\begin{array}{cc}
x_{1} & -x_{2}^{*} \\
x_{2} & x_{1}^{*}
\end{array}\right] .
\end{aligned}
$$

Using the new vectorization (2.3), which preserves the redundancy in the STBC:

$$
\mathbf{c}^{\prime}=\left(\Re\left\{x_{1}\right\}, \Re\left\{x_{2}\right\}, \Im\left\{x_{1}\right\}, \Im\left\{x_{2}\right\},-\Re\left\{x_{2}\right\}, \Re\left\{x_{1}\right\}, \Im\left\{x_{2}\right\},-\Im\left\{x_{1}\right\}\right) .
$$

If the underlying constellation is BPSK, then the minimum distance between any two vectorized codeword matrices is 2 . Therefore, the new vectorization provides an equivalent concept of redundancy. For an OSTBC with BPSK, (2.4) is reduced to

$$
M(\mathbf{C})=-2 \sum_{k=1}^{L} \sum_{j=1}^{n_{T}} \Re\left\{\left\langle\mathbf{r}_{k}, \mathbf{h}_{j}\right\rangle\right\} \Re\left\{c_{j k}\right\} .
$$

Therefore, the ML codeword matrix is that which satisfies

$$
M(\hat{\mathbf{C}})=\arg \underset{\text { all } \mathbf{C}}{\max }\left\{\sum_{k=1}^{L} \sum_{j=1}^{n_{T}} \Re\left\{\left\langle\mathbf{r}_{k}, \mathbf{h}_{j}\right\rangle\right\} \Re\left\{c_{j k}\right\}\right\} .
$$

This is the same form as 4.9). Therefore, the inner product, $\Re\left\{\left\langle\mathbf{r}_{k}, \mathbf{h}_{j}\right\rangle\right\}$, can be interpreted as an equivalent of a reliability measure for $c_{j k}$, and a codeword test can 
be developed by exactly paralleling the derivation in Section 4.3.1. Although this parallel is interesting, it is of no practical value: one always performs ML decoding for OSTBCs, and hence there is never a sub-optimal guess to apply to the codeword test. Therefore, we need to consider non-orthogonal STBCs with at least 4-QAM to derive any practical results. Assuming an equi-energy underlying constellation, 2.4 reduces to

$$
\begin{aligned}
M(\mathbf{C})= & \sum_{j=1}^{n_{T}} \sum_{m=j+1}^{n_{T}}\left(\Re\left\{\left\langle\mathbf{h}_{j}, \mathbf{h}_{m}\right\rangle\right\} \Re\left\{\left\langle\mathbf{c}^{(m)}, \mathbf{c}^{(j)}\right\rangle\right\}+\Im\left\{\left\langle\mathbf{h}_{j}, \mathbf{h}_{m}\right\rangle\right\} \Im\left\{\left\langle\mathbf{c}^{(m)}, \mathbf{c}^{(j)}\right\rangle\right\}\right) \\
& -\sum_{k=1}^{L} \sum_{j=1}^{n_{T}}\left(\Re\left\{\left\langle\mathbf{r}_{k}, \mathbf{h}_{j}\right\rangle\right\} \Re\left\{c_{j k}\right\}+\Im\left\{\left\langle\mathbf{r}_{k}, \mathbf{h}_{j}\right\rangle\right\} \Im\left\{c_{j k}\right\}\right) .
\end{aligned}
$$

To develop a codeword test paralleling the classic development in Section 4.3.1, we need to find a useful (i.e. tight enough) upper bound on (4.12). This is further complicated due to the coupling term in the metric introduced by the non-orthogonality in the STBC. Deriving this bound is a challenging and open problem.

Another approach to developing a codeword test criterion for the MIMO system is to express the reliability values in terms of probabilities, as in [37, Section VI], and then parallel the development in [37, Section VI]. Applying this approach to the MIMO scenario results in similar challenges due to the coupling introduced by non-orthogonal STBCs.

\subsubsection{Conclusion}

As discussed above, developing OTC for MIMO systems that employ space-time block coding is a challenging topic. Currently, there are no works published in the area. Despite the challenges discussed above, the new vectorization and metric expression 
provide a promising framework: the new vectorization uses redundancy in the transmission matrix to provide a quantifiable definition of minimum distance for a STBC, and the inner products in the coupled and decoupled terms in (2.4) act as reliabilities that can be bounded.

\subsection{Conclusions}

This chapter has summarized the contributions of the thesis and has highlighted some of the future work of interest. In particular, the following two open problems resulting from the work have been considered in detail: (1) how can one analytically justify the optimum rotation angle for the new QOSTBC in (2.5), and (2) how can one use the new vectorization and metric expression to develop useful OTC for STBCs.

Not surprisingly, this thesis has answered some of the questions of interest to current researchers in the field, but in doing so has left new unanswered questions. 


\section{Proofs}

\section{Proof of Lemma 1:}

Note that $\mathbf{r}^{\prime} \equiv \mathbf{y}^{\prime}$, i.e., the coordinates of the vector $\mathbf{y}^{\prime}$ are a permutation of the coordinates of the vector $\mathbf{r}^{\prime}$. A permutation of the vector positions does not change the Euclidean norm, therefore $\left\|\mathbf{r}^{\prime}\right\|^{2}=\left\|\mathbf{y}^{\prime}\right\|^{2}$.

To show $\left\|\mathbf{F}^{\prime} \mathbf{x}^{\prime}\right\|^{2}=\left\|\mathbf{G}^{\prime} \mathbf{c}^{\prime}\right\|^{2}=\|\mathbf{H C}\|^{2}$ : Using the standard vectorization, it can easily be shown that $\left\|\mathbf{F}^{\prime} \mathbf{x}^{\prime}\right\|^{2}=\left\|\mathbf{F x}^{\prime}\right\|^{2}$. Since $\mathbf{F} \mathbf{x}^{\prime}=\operatorname{vec}(\mathbf{H C})$ by construction, therefore $\left\|\mathbf{F}^{\prime} \mathbf{x}^{\prime}\right\|^{2}=\left\|\mathbf{F} \mathbf{x}^{\prime}\right\|^{2}=\|\operatorname{vec}(\mathbf{H C})\|^{2}=\|\mathbf{H C}\|^{2}$. Expanding $\|\mathbf{H C}\|^{2}$ we have:

$$
\begin{aligned}
\|\mathbf{H C}\|^{2}= & \sum_{k=1}^{L} \sum_{i=1}^{n_{R}}\left|\sum_{j=1}^{n_{T}} h_{i j} c_{j k}\right|^{2} \\
= & \sum_{k=1}^{L} \sum_{i=1}^{n_{R}}\left[\left(\sum_{j=1}^{n_{T}}\left(\Re\left\{h_{i j}\right\} \Re\left\{c_{j k}\right\}-\Im\left\{h_{i j}\right\} \Im\left\{c_{j k}\right\}\right)\right)^{2}\right. \\
& \left.+\left(\sum_{j=1}^{n_{T}}\left(\Re\left\{h_{i j}\right\} \Im\left\{c_{j k}\right\}+\Im\left\{h_{i j}\right\} \Re\left\{c_{j k}\right\}\right)\right)^{2}\right] \\
= & \sum_{k=1}^{L}\left\|\mathbf{H}^{\prime} \mathbf{c}_{k}^{\prime}\right\|^{2}=\left\|\mathbf{G}^{\prime} \mathbf{c}^{\prime}\right\|^{2} .
\end{aligned}
$$

Finally, to show $\mathbf{r}^{\prime T} \mathbf{F}^{\prime} \mathbf{x}^{\prime}=\mathbf{y}^{\prime T} \mathbf{G}^{\prime} \mathbf{c}^{\prime}$ : Define $P(\cdot)$ as a permutation that acts on any real matrix with $2 n_{R} L$ rows. $P(\cdot)$ interchanges the rows of the matrix such that if $P(\cdot)$ were applied to $\mathbf{r}^{\prime}$, the permutation would yield $\mathbf{y}^{\prime}$, i.e., $\mathbf{y}^{\prime}=P\left(\mathbf{r}^{\prime}\right)$. Since addition is a commutative operation, for any real vector $\mathbf{b}$ of dimension $2 n_{R} L \times 1$ we 
have

$$
\mathbf{r}^{\prime T} \mathbf{b}=\mathbf{y}^{\prime T} P(\mathbf{b})
$$

Note that both $\mathbf{F}^{\prime} \mathbf{x}^{\prime}$ and $\mathbf{G}^{\prime} \mathbf{c}^{\prime}$ result in vectors of dimension $2 n_{R} L \times 1$. Also $P\left(\mathbf{F}^{\prime} \mathbf{x}^{\prime}\right)=$ $P\left(\mathbf{F}^{\prime}\right) \mathbf{x}^{\prime}$

$P\left(\mathbf{F}^{\prime}\right)$ can be partitioned along the rows as follows:

$$
P\left(\mathbf{F}^{\prime}\right)=\left[\begin{array}{c}
\mathbf{A}_{1} \\
\mathbf{A}_{2} \\
\vdots \\
\mathbf{A}_{L}
\end{array}\right]
$$

where $\mathbf{A}_{k}$ is a matrix of dimension $2 n_{R} \times 2 n_{s}$. Therefore,

$$
P\left(\mathbf{F}^{\prime} \mathbf{x}^{\prime}\right)=P\left(\mathbf{F}^{\prime}\right) \mathbf{x}^{\prime}=\left[\begin{array}{c}
\mathbf{A}_{1} \\
\mathbf{A}_{2} \\
\vdots \\
\mathbf{A}_{L}
\end{array}\right] \mathbf{x}^{\prime}=\left[\begin{array}{cccc}
\mathbf{A}_{1} & \mathbf{0} & \ldots & \mathbf{0} \\
\mathbf{0} & \mathbf{A}_{2} & \ldots & \mathbf{0} \\
\vdots & \vdots & \vdots & \vdots \\
0 & \mathbf{0} & \ldots & \mathbf{A}_{L}
\end{array}\right]\left[\begin{array}{c}
\mathbf{x}^{\prime} \\
\mathbf{x}^{\prime} \\
\vdots \\
\mathbf{x}^{\prime}
\end{array}\right]=\mathbf{G}^{\prime} \mathbf{c}^{\prime}
$$

Therefore, $P\left(\mathbf{F}^{\prime} \mathbf{x}^{\prime}\right)=\mathbf{G}^{\prime} \mathbf{c}^{\prime}$. Letting $\mathbf{b}=\mathbf{F}^{\prime} \mathbf{x}^{\prime}$ in (13) yields:

$$
\mathbf{r}^{\prime T} \mathbf{F}^{\prime} \mathbf{x}^{\prime}=\mathbf{y}^{\prime T} \mathbf{G}^{\prime} \mathbf{c}^{\prime}
$$

Proof of Lemma 2:

Expanding the ML metric $\left\|\mathbf{y}^{\prime}-\mathbf{G}^{\prime} \mathbf{c}^{\prime}\right\|^{2}=\left\|\mathbf{y}^{\prime}\right\|^{2}+\left\|\mathbf{G}^{\prime} \mathbf{c}^{\prime}\right\|^{2}-2 \mathbf{y}^{\prime T} \mathbf{G}^{\prime} \mathbf{c}^{\prime}$, the term $\mathbf{y}^{\prime T} \mathbf{G}^{\prime} \mathbf{c}^{\prime}$ can be expanded as follows: 


$$
\begin{aligned}
\mathbf{y}^{\prime T} \mathbf{G}^{\prime} \mathbf{c}^{\prime}= & {\left[\mathbf{r}_{1}^{\prime T} \mathbf{r}_{2}^{\prime T} \ldots \mathbf{r}_{L}^{\prime T}\right] \mathbf{G}^{\prime}\left[\mathbf{c}_{1}^{\prime T} \mathbf{c}_{2}^{\prime T} \ldots \mathbf{c}_{L}^{\prime T}\right]^{T}=\sum_{k=1}^{L} \mathbf{r}_{k}^{\prime T}\left(\mathbf{H}^{\prime} \mathbf{c}_{k}^{\prime}\right) } \\
= & \sum_{k=1}^{L}\left[\sum_{i=1}^{n_{R}} \Re\left\{r_{i k}\right\} \sum_{j=1}^{n_{T}}\left(\Re\left\{h_{i j}\right\} \Re\left\{c_{j k}\right\}-\Im\left\{h_{i j}\right\} \Im\left\{c_{j k}\right\}\right)\right. \\
& \left.+\sum_{i=1}^{n_{R}} \Im\left\{r_{i k}\right\} \sum_{j=1}^{n_{T}}\left(\Im\left\{h_{i j}\right\} \Re\left\{c_{j k}\right\}+\Re\left\{h_{i j}\right\} \Im\left\{c_{j k}\right\}\right)\right] \\
= & \left.\sum_{k=1}^{L} \sum_{j=1}^{n_{T}} \Re\left\{c_{j k}\right\} \sum_{i=1}^{n_{R}}\left(\Re\left\{r_{i k}\right\} \Re\left\{h_{i j}\right\}+\Im\left\{r_{i k}\right\} \Im\left\{h_{i j}\right\}\right\}\right) \\
& \left.+\sum_{k=1}^{L} \sum_{j=1}^{n_{T}} \Im\left\{c_{j k}\right\} \sum_{i=1}^{n_{R}}\left(\Im\left\{r_{i k}\right\} \Re\left\{h_{i j}\right\}-\Re\left\{r_{i k}\right\} \Im\left\{h_{i j}\right\}\right\}\right) \\
= & \sum_{k=1}^{L} \sum_{j=1}^{n_{T}}\left(\Re\left\{\left\langle\mathbf{r}_{k}, \mathbf{h}_{j}\right\rangle\right\} \Re\left\{c_{j k}\right\}+\Im\left\{\left\langle\mathbf{r}_{k}, \mathbf{h}_{j}\right\rangle\right\} \Im\left\{c_{j k}\right\}\right) .
\end{aligned}
$$

The term $\left\|\mathbf{G}^{\prime} \mathbf{c}^{\prime}\right\|^{2}=\|\mathbf{H C}\|^{2}$ can also be expanded as follows:

$$
\begin{aligned}
\|\mathbf{H C}\|^{2}= & \sum_{k=1}^{L} \sum_{i=1}^{n_{R}}\left|\sum_{j=1}^{n_{T}} h_{i j} c_{j k}\right|^{2} \\
= & \sum_{k=1}^{L} \sum_{i=1}^{n_{R}}\left[\left(\sum_{j=1}^{n_{T}}\left(\Re\left\{h_{i j}\right\} \Re\left\{c_{j k}\right\}-\Im\left\{h_{i j}\right\} \Im\left\{c_{j k}\right\}\right)\right)^{2}\right. \\
& \left.+\left(\sum_{j=1}^{n_{T}}\left(\Re\left\{h_{i j}\right\} \Im\left\{c_{j k}\right\}+\Im\left\{h_{i j}\right\} \Re\left\{c_{j k}\right\}\right)\right)^{2}\right] .
\end{aligned}
$$

Using

$$
\left(\sum_{j=1}^{n_{T}} a_{j}\right)^{2}=\sum_{j=1}^{n_{T}} a_{j}^{2}+2 \sum_{j=1}^{n_{T}} \sum_{m=j+1}^{n_{T}} a_{j} a_{m}
$$


we have $\left(\sum_{j=1}^{n_{T}}\left(\Re\left\{h_{i j}\right\} \Re\left\{c_{j k}\right\}-\Im\left\{h_{i j}\right\} \Im\left\{c_{j k}\right\}\right)\right)^{2}=$

$$
\begin{aligned}
& \sum_{j=1}^{n_{T}}\left[\Re\left\{h_{i j}\right\}^{2} \Re\left\{c_{j k}\right\}^{2}+\Im\left\{h_{i j}\right\}^{2} \Im\left\{c_{j k}\right\}^{2}-2 \Re\left\{h_{i j}\right\} \Im\left\{h_{i j}\right\} \Re\left\{c_{j k}\right\} \Im\left\{c_{j k}\right\}\right. \\
& +2 \sum_{m=j+1}^{n_{T}}\left(\Re\left\{h_{i j}\right\} \Re\left\{h_{i m}\right\} \Re\left\{c_{j k}\right\} \Re\left\{c_{m k}\right\}-\Re\left\{h_{i j}\right\} \Im\left\{h_{i m}\right\} \Re\left\{c_{j k}\right\} \Im\left\{c_{m k}\right\}\right. \\
& \left.\left.-\Im\left\{h_{i j}\right\} \Re\left\{h_{i m}\right\} \Im\left\{c_{j k}\right\} \Re\left\{c_{m k}\right\}\right)+\Im\left\{h_{i j}\right\} \Im\left\{h_{i m}\right\} \Im\left\{c_{j k}\right\} \Im\left\{c_{m k}\right\}\right] .
\end{aligned}
$$

Expanding $\left(\sum_{j=1}^{n_{T}}\left(\Re\left\{h_{i j}\right\} \Im\left\{c_{j k}\right\}+\Im\left\{h_{i j}\right\} \Re\left\{c_{j k}\right\}\right)\right)^{2}$ in a similar fashion and substituting into the original expression yields $\|\mathbf{H C}\|^{2}$ :

$$
\begin{aligned}
= & \sum_{k=1}^{L} \sum_{i=1}^{n_{R}} \sum_{j=1}^{n_{T}}\left[\Re\left\{h_{i j}\right\}^{2} \Re\left\{c_{j k}\right\}^{2}+\Im\left\{h_{i j}\right\}^{2} \Im\left\{c_{j k}\right\}^{2}+\Im\left\{h_{i j}\right\}^{2} \Re\left\{c_{j k}\right\}^{2}+\Re\left\{h_{i j}\right\}^{2} \Im\left\{c_{j k}\right\}^{2}\right. \\
& +2 \sum_{m=j+1}^{n_{T}}\left(\Re\left\{h_{i j}\right\} \Re\left\{h_{i m}\right\} \Re\left\{c_{j k}\right\} \Re\left\{c_{m k}\right\}-\Re\left\{h_{i j}\right\} \Im\left\{h_{i m}\right\} \Re\left\{c_{j k}\right\} \Im\left\{c_{m k}\right\}\right. \\
& -\Im\left\{h_{i j}\right\} \Re\left\{h_{i m}\right\} \Im\left\{c_{j k}\right\} \Re\left\{c_{m k}\right\}+\Im\left\{h_{i j}\right\} \Im\left\{h_{i m}\right\} \Im\left\{c_{j k}\right\} \Im\left\{c_{m k}\right\} \\
& +\Im\left\{h_{i j}\right\} \Im\left\{h_{i m}\right\} \Re\left\{c_{j k}\right\} \Re\left\{c_{m k}\right\}+\Im\left\{h_{i j}\right\} \Re\left\{h_{i m}\right\} \Re\left\{c_{j k}\right\} \Im\left\{c_{m k}\right\} \\
& \left.\left.+\Re\left\{h_{i j}\right\} \Im\left\{h_{i m}\right\} \Im\left\{c_{j k}\right\} \Re\left\{c_{m k}\right\}+\Re\left\{h_{i j}\right\} \Re\left\{h_{i m}\right\} \Im\left\{c_{j k}\right\} \Im\left\{c_{m k}\right\}\right)\right] \\
= & \sum_{k=1}^{L} \sum_{i=1}^{n_{R}} \sum_{j=1}^{n_{T}}\left(\Re\left\{h_{i j}\right\}^{2} \Re\left\{c_{j k}\right\}^{2}+\Im\left\{h_{i j}\right\}^{2} \Im\left\{c_{j k}\right\}^{2}+\Im\left\{h_{i j}\right\}^{2} \Re\left\{c_{j k}\right\}^{2}+\Re\left\{h_{i j}\right\}^{2} \Im\left\{c_{j k}\right\}^{2}\right) \\
& +2 \sum_{j=1}^{n_{T}} \sum_{m=j+1}^{n_{T}}\left(\Re\left\{\left\langle\mathbf{h}_{j}, \mathbf{h}_{m}\right\rangle\right\} \Re\left\{\left\langle\mathbf{c}^{(m)}, \mathbf{c}^{(j)}\right\rangle\right\}+\Im\left\{\left\langle\mathbf{h}_{j}, \mathbf{h}_{m}\right\rangle\right\} \Im\left\{\left\langle\mathbf{c}^{(m)}, \mathbf{c}^{(j)}\right\rangle\right\}\right) \\
= & \sum_{j=1}^{n_{T}}\left\|\mathbf{h}_{j}\right\|^{2}\left\|\mathbf{c}^{(j)}\right\|^{2} \\
& +2 \sum_{j=1}^{n_{T}} \sum_{m=j+1}^{n_{T}}\left(\Re\left\{\left\langle\mathbf{h}_{j}, \mathbf{h}_{m}\right\rangle\right\} \Re\left\{\left\langle\mathbf{c}^{(m)}, \mathbf{c}^{(j)}\right\rangle\right\}+\Im\left\{\left\langle\mathbf{h}_{j}, \mathbf{h}_{m}\right\rangle\right\} \Im\left\{\left\langle\mathbf{c}^{(m)}, \mathbf{c}^{(j)}\right\rangle\right\}\right) .
\end{aligned}
$$


Therefore, $\left\|\mathbf{y}^{\prime}-\mathbf{G}^{\prime} \mathbf{c}^{\prime}\right\|^{2}$

$$
\begin{aligned}
= & \left\|\mathbf{y}^{\prime}\right\|^{2}+\sum_{j=1}^{n_{T}}\left\|\mathbf{h}_{j}\right\|^{2}\left\|\mathbf{c}^{(j)}\right\|^{2} \\
& +2 \sum_{j=1}^{n_{T}} \sum_{m=j+1}^{n_{T}}\left(\Re\left\{\left\langle\mathbf{h}_{j}, \mathbf{h}_{m}\right\rangle\right\} \Re\left\{\left\langle\mathbf{c}^{(m)}, \mathbf{c}^{(j)}\right\rangle\right\}+\Im\left\{\left\langle\mathbf{h}_{j}, \mathbf{h}_{m}\right\rangle\right\} \Im\left\{\left\langle\mathbf{c}^{(m)}, \mathbf{c}^{(j)}\right\rangle\right\}\right) \\
& -2 \sum_{k=1}^{L} \sum_{j=1}^{n_{T}}\left(\Re\left\{\left\langle\mathbf{r}_{k}, \mathbf{h}_{j}\right\rangle\right\} \Re\left\{c_{j k}\right\}+\Im\left\{\left\langle\mathbf{r}_{k}, \mathbf{h}_{j}\right\rangle\right\} \Im\left\{c_{j k}\right\}\right) .
\end{aligned}
$$

Removing all components independent of the transmission matrix yields:

$$
\begin{aligned}
M(\mathbf{C})= & 2 \sum_{j=1}^{n_{T}} \sum_{m=j+1}^{n_{T}}\left(\Re\left\{\left\langle\mathbf{h}_{j}, \mathbf{h}_{m}\right\rangle\right\} \Re\left\{\left\langle\mathbf{c}^{(m)}, \mathbf{c}^{(j)}\right\rangle\right\}+\Im\left\{\left\langle\mathbf{h}_{j}, \mathbf{h}_{m}\right\rangle\right\} \Im\left\{\left\langle\mathbf{c}^{(m)}, \mathbf{c}^{(j)}\right\rangle\right\}\right) \\
& +\sum_{j=1}^{n_{T}}\left\|\mathbf{h}_{j}\right\|^{2}\left\|\mathbf{c}^{(j)}\right\|^{2}-2 \sum_{k=1}^{L} \sum_{j=1}^{n_{T}}\left(\Re\left\{\left\langle\mathbf{r}_{k}, \mathbf{h}_{j}\right\rangle\right\} \Re\left\{c_{j k}\right\}+\Im\left\{\left\langle\mathbf{r}_{k}, \mathbf{h}_{j}\right\rangle\right\} \Im\left\{c_{j k}\right\}\right) .
\end{aligned}
$$




\section{Bibliography}

[1] D. Tse and P. Viswanath, Fundamentals of Wireless Communication, Cambridge, U.K.: Cambridge University Press, 2005.

[2] E. Biglieri, J. Proakis, and S. Shamai, , "Fading channels: information-theoretic and communications aspects," IEEE Trans. Inf. Theory, vol. 44, pp. 2619-2692, Oct. 1998.

[3] S. M. Alamouti, "A simple transmit diversity technique for wireless communications," IEEE J. Sel. Areas Commun., vol. 16, pp. 1451-1458, Oct. 1998.

[4] G. J. Foschini, "Layered space-time architecture for wireless communication in a fading environment when using multi-element antennas," Bell Labs. Tech. J., vol. 1, no. 2, pp. 4159, 1996.

[5] H. Jafarkhani, Space-Time Coding: Theory and Practice, Cambridge, U.K.: Cambridge University Press,, 2005.

[6] B. Hassibi and M. Hochwald, "High-rate codes that are linear in space and time," IEEE Trans. Inf. Theory, vol. 48, pp. 1804-1824, July 2002. 
[7] L. Zheng and D. N. C. Tse, "Diversity and multiplexing: a fundamental tradeoff in multiple-antenna channels," IEEE Trans. Inf. Theory, vol. 49, pp. 1073-1096, May 2003.

[8] E. Larsson and P. Stoica, Space-Time Block Coding for Wireless Communications, Cambridge, U.K.: Cambridge University Press, 2003.

[9] V. Tarokh, H. Jafarkhani, and A. R. Calderbank, "Space-time block codes from orthogonal designs," IEEE Trans. Inf. Theory, vol. 45, pp. 1456-1467, July 1999.

[10] H. Jafarkhani, "A quasi-orthogonal space-time block code," IEEE Trans. Commun., vol. 49, pp. 1-4, Jan. 2001.

[11] L. Xian and H. Liu, "Rate-one space-time block codes with full diversity," IEEE Trans. Commun., vol. 53, pp. 1986-1990, Dec. 2005.

[12] C. Yuen, Y. L. Guan, and T. T. Tjhung, "Quasi-orthogonal STBC with minimum decoding complexity," IEEE Trans. Wireless Commun., vol. 4, pp. 20892094, Sept. 2005.

[13] N. Sharma and C. B. Papadias, "Improved quasi-orthogonal codes through constellation rotation," IEEE Trans. Commun., vol. 51, pp. 332-335, Mar. 2003.

[14] W. Su and X.-G. Xia, "Signal constellations for quasi-orthogonal space-time block codes with full-diversity," IEEE Trans. Inf. Theory, vol. 48, pp. 23312347, Oct. 2004.

[15] B. Hassibi and H. Vikalo, "On the sphere-decoding algorithm 1. Expected complexity," IEEE Trans. Signal Process., vol. 53, pp. 2806-2818, Apr. 2005. 
[16] A. K. Lenstra, H. W. Lenstra, and L. Lovsz, "Factoring polynomials with rational coefficients," Math. Ann., pp. 515534, 1982.

[17] M. O. Damen, H. El Gamal, and G. Caire, "On maximum-likelihood detection and the search for the closest lattice point," IEEE Trans. Inf. Theory, vol. 49, pp. 2389-2402, Oct. 2003.

[18] O. Damen, A. Chkeif, and J.-C. Belfiore, "Lattice code decoder for space-time codes," IEEE Commun. Lett., vol. 4, pp. 161-163, May 2000.

[19] E. Viterbo and J. Boutros, "A universal lattice code decoder for fading channels," IEEE Trans. Inf. Theory, vol. 45, pp. 1639-1642, July 1999.

[20] J. Jalden and B. Ottersten, "On the complexity of sphere decoding in digital communications," IEEE Trans. Signal Process., vol. 53, pp. 1474-1484, Apr. 2005.

[21] U. Fincke and M. Pohst, "Improved methods for calculating vectors of short length in a lattice, including a complexity analysis," Math. Comput., vol. 44, pp. 463-471, Apr. 1985.

[22] C. P. Schnorr and M. Euchner, "Lattice basis reduction: Improved practical algorithms and solving subset sum problems," Math. Programming,, vol. 66, pp. 181-191, 1994.

[23] E. Agrell, T. Eriksson, A. Vardy, and K. Zeger, "Closest point search in lattices," IEEE Trans. Inf. Theory, vol. 48, pp. 2201-2214, Aug 2002. 
[24] A. M. Chan and I. Lee, "A new reduced-complexity sphere decoder for multiple antenna systems," IEEE International Conference on Communications, pp. 460-464, 2002.

[25] A. Peng, I.-M. Kim, and S. Yousefi, "Low-complexity sphere decoding algorithm for quasi-orthogonal space-time block codes," IEEE Trans. Commun., vol. 54, pp. 377-382, Mar. 2006.

[26] M. Stojnic, H. Vikalo, and B. Hassibi, "Further results on speeding up the sphere decoder," IEEE International Conference on Acoustics, Speech, and Signal Processing, pp. 549-552, 2006.

[27] K. Su and I. J. Wassell, "A new ordering for efficient sphere decoding," IEEE International Conference on Communications, pp. 1906-1910, 2005.

[28] W. Xu, Y. Wang, Z. Z. Jing, and J. Wang, "A computationally efficient exact ML sphere decoder," IEEE Global Telecommunications Conference, pp. 2594$2598,2004$.

[29] Z. A. Khan and B. S. Rajan, "Single-symbol maximum-likelihood decodable linear STBCs," IEEE Trans. Inf. Theory, vol. 52, pp. 2062-2091, May 2006.

[30] G. H. Golub and C. F. Van Loan, Matrix Computations. Baltimore, MD: John Hopkins University Press, 1983.

[31] M.-T. Le, V.-S. Pham, L. Mai, and G. Yoon, "Very-low complexity maximumlikelhihood decoders for four-transmit-antenna quasi-orthogonal space-time code," IEICE Trans. Commun., vol. E88-B, pp. 3802-3805, Sept. 2005. 
[32] V.-S. Pham, M.-T. Le, L. Mai, and G. Yoon, "Low-complexity MMSE-QRDbased ML decoder for quasi-orthogonal STBC scheme in MIMO wireless communications systems with four-transmit antennas," ICACT 2006, pp. 19371939, Feb. 2006.

[33] A. Sezgin, E. A. Jorswieck, and E. Costa, "Lattice-reduction aided detection: spatial multiplexing versus quasi-orthogonal STBC," IEEE Vehicular Technology Conference, pp. 2389-2393, 2004.

[34] M. Rupp, C. Mecklenbrauker, and G. Gritsch, "High diversity with simple space time block-codes and linear receivers ," GLOBECOM 2003, pp. 302-306, Dec. 2003.

[35] H. Yao and G. W. Wornell, "Lattice-reduction-aided detectors for MIMO communication systems," IEEE Global Telecommunications Conference, pp. 424$428,2002$.

[36] D. Chase, "A class of algorithms for decoding block codes with channel measurement information," IEEE Trans. Inf. Theory, vol. IT-18, pp. 170-182, Jan. 1972.

[37] T. Kaneko, T. Nishijima, H. Inazumi, and S. Hirasawa, "An efficient maximumlikelihood-decoding algorithm for linear block codes with algebraic decoder," IEEE Trans. Inf. Theory, vol. 40, pp. 320-327, Mar. 1994.

[38] J. Leuschner and S. Yousefi, "A new generic maximum-likelihood metric expression for space-time block codes with applications to decoding," 2007 Conference 
on Information Sciences and Systems, Johns Hopkins University, Baltimore, MD, Mar. 2007.

[39] J. Leuschner and S. Yousefi, "A new generic maximum-likelihood metric expression for space-time block codes with applications to decoding," IEEE Trans. Inf. Theory, revised.

[40] J. Leuschner and S. Yousefi, "On the ML decoding of quasi-orthogonal spacetime block codes via sphere decoding and exhaustive search," 2007 International Wireless Communications and Mobile Computing Conference, Honolulu, Hawaii, Aug. 2007.

[41] J. Leuschner and S. Yousefi, "On the ML decoding of quasi-orthogonal spacetime block codes via sphere decoding and exhaustive search," IEEE Trans. Wireless Commun., under review.

[42] J. Leuschner and S. Yousefi, "A new sub-optimal decoder for quasi-orthogonal space-time block codes," IEEE Commun. Lett., under review.

[43] D. Wang and X.-G. Xia, "Optimal diversity product rotations for quasiorthogonal STBC with MPSK symbols," IEEE Trans. Commun., vol. 9, pp. 420-422, May 2005.

[44] C. Yuen, Y. L. Guan, and T. T. Tjhung, "Quasi-orthogonal STBC with minimum decoding complexity: Further results," in Proc. Wireless Communications and Networking Conf. (WCNC), New Orleans, LA, 2005, pp. 483-488. 
[45] H. Jafarkhani and N. Hassanpour, "Super-quasi-orthogonal space-time trellis codes for four transmit antennas," IEEE Trans. Wireless Commun., vol. 4, pp. 215-227, Jan. 2005.

[46] A. Sezgin, E. A. Jorswieck, and H. Boche, "Performance criteria analysis and further performance results for quasi-orthogonal space-time block codes," ISSPIT 2003, pp. 345-348, Dec. 2003.

[47] L. Xian and H. Liu, "Optimal rotation angles for quasi-orthogonal space-time codes with PSK modulation," IEEE Trans. Commun., vol. 51, pp. 332-335, Mar. 2003.

[48] Z. A. Khan and B. S. Rajan, "Space-time block codes from co-ordinate interleaved orthogonal designs," in Proc. IEEE ISIT 2002, Switzerland, 2002, pp. 275.

[49] Z. A. Khan, B. S. Rajan, and M. H. Lee, "Rectangular co-ordinate interleaved orthogonal designs," in Proc. IEEE GLOBECOM 2003, pp. 2004-2009, Dec. 2003.

[50] S. Lin and D.J. Costello Jr., Error Control Coding, 2nd Ed., New Jersey: Prentice Hall, 2004.

[51] P. F. Swaszek and W. Jones, "How often is hard-decision decoding enough?," IEEE Trans. Inf. Theory, vol. 44, pp. 1187-1193, Mar. 1998.

[52] D. J. Taipale and M. B. Pursley, "An improvement to generalized-minimumdistance decoding," IEEE Trans. Inf. Theory, vol. 37, pp. 167-172, Jan. 1991. 